\title{
ANÁLISE DE GRUPOS DE EXPERIMENTOS COM HETEROGENEIDADE DE VARIÂNCIAS
}

\author{
WILSON ALVES DE OLIVEIRA \\ Estatístico
}

Orientadora: Prof ${ }^{2}$. $\mathrm{Dr}^{\mathrm{a}}$. MARIA CRISTINA S. NOGUEIRA

Dissertação apresentada à Escola Superior de Agricultura "Luiz de Queiroz", Universidade de São Paulo, para obtenção do título de mestre em Agronomia, Área de Concentração: Estatística e Experimentação Agronômica.

P I R A C I C A B A

Estado de São Paulo - Brasil

Maio - 1999 
Dados Internacionais de Catalogação na Publicação (CIP)

DIVISÃO DE BIBLIOTECA E DOCUMENTAÇÃO - Campus "Luiz de Queiroz"/USP

Oliveira, Wilson Alves de Análise de grupos de experimentos com heterogeneidade de variâncias / Wilson Alves de Oliveira. - - Piracicaba, 1999.

$97 \mathrm{p}$.

Dissertação (mestrado) - - Escola Superior de Agricultura Luiz de Queiroz, 1999. Bibliografia.

1. Análise de variância 2. Erro experimental 3. Estatistica aplicada 4. Experimento agricola 5. Interação 6. Residuo I. Título

CDD 519.535

"Permitida a cópta total ou parcial deste doenmento, desde que citada a fonte - 0 Antor" 


\section{Dedico,}

À minha querida esposa, Irene, pelo incentivo e companheirismo, em todos os momentos. 


\title{
AGRADECIMENTOS
}

\section{À DEUS, por mostrar caminhos nas horas mais dificeis.}

\author{
À Prof . Dr ${ }^{\mathrm{a}}$. Maria Cristina Stolf Nogueira, pela valiosa orientação no \\ desenvolvimento deste trabalho;
}

Ao Prof. Dr. Décio Barbin, pela amizade e inestimável contribuição na elaboração deste trabalho;

Ao Prof. Dr. Armando Conagin, pelas sugestões que possibilitaram o enriquecimento deste trabalho;

Ao amigo Cristián Andrés Carranza, pela contribuição oferecida para a realização deste trabalho;

Aos amigos do Programa de Pós-graduação, pela convivência e amizade durante este período e em especial aos colegas de curso, Adilson, Afrânio, Idemauro, Jeanete, Maria Helena e Sandra;

Aos professores do Departamento de Ciências Exatas da Escola Superior de Agricultura Luiz de Queiroz, pela transmissão dos conhecimentos;

Aos funcionários do Departamento de Ciências Exatas da Escola Superior de Agricultura Luiz de Queiroz, pela atenção e dedicação; 
À Universidade Estadual do Oeste do Paraná - UNIOESTE e aos professores do Departamento de Matemática e Estatística pela oportunidade e incentivo;

Aos meus pais, Ezequiel e Delfina, pela educação, oportunidade na realização do curso de graduação e incentivo para Pós-graduação;

Aos meus irmãos, pela amizade e incentivo;

Ả Evelyn Tânia e Esloany Daisy, minhas enteadas, pela compreensão;

À CAPES pelos recursos financeiros;

À todos àqueles que de algum modo colaboraram para a realização deste trabalho. 


\section{SUMÁRIO}

Página

LISTA DE TABELAS ........................ vii

RESUMO ............................. viii

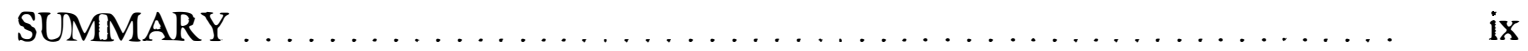

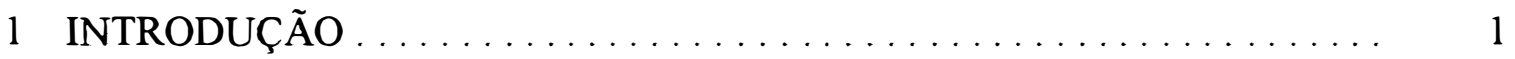

2 REVISÃO DE LITERATURA . . . . . . . . . . . . . . . 3

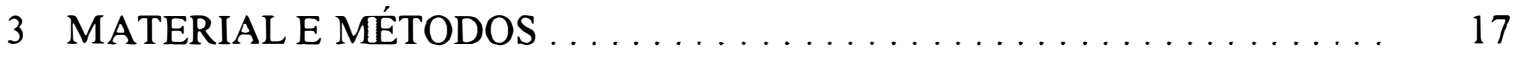

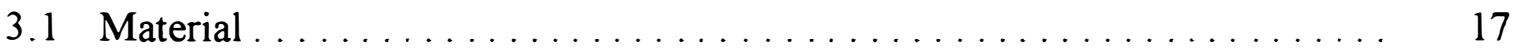

3.2 Métodos . . . . . . . . . . . . . . . . . . . . . . . . 18

3.2.1 Testes para homogeneidade de variâncias . . . . . . . . . . . . 22

3.2.1.1 Teste de Hartley . . . . . . . . . . . . . . . . . . . . . 22

3.2.1.2 Teste de Bartlett . . . . . . . . . . . . . . . . . . . . 22

3.2 .2 Resíduo específico . . . . . . . . . . . . . . . . . 23

3.2.3 Método de Cochran para ajuste dos graus de liberdade . . . . . . . . . . . 24

3.2 .4 Teste de combinação de probabilidades . . . . . . . . . . . . . . 25

3.2.4.1 Combinação de resultados de testes individuais na análise de grupos de experimentos . . . . . . . . . . . . . . . . 25

3.2.4.2 Teorema da combinação de probabilidades . . . . . . . . . . . . . 26

3.2.5 Análise de variância ponderada . . . . . . . . . . . . . . . . . 29

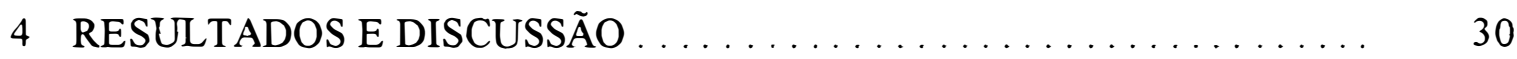

4.1 Esperança dos quadrados médios . . . . . . . . . . . . . . . . . . . 30

4.1.1 Esperança dos quadrados médios para os componentes lineares e quadráticos de $\mathrm{N}, \mathrm{P}$ e K . . . . . . . . . . . . . . . . 38

4.1.2 Esperança dos quadrados médios para $\mathrm{N}^{\prime} \times \mathrm{P}^{\prime}, \mathrm{N}^{\prime} \times \mathrm{K}^{\prime}$ e $\mathrm{P}^{\prime} \times \mathrm{K}^{\prime} \ldots \ldots .43$ 
4.1.2.1 Obtenção da $\mathrm{E}\left[\mathrm{QM}\left(\mathrm{N}^{\prime} \times \mathrm{P}^{\prime}\right)\right] \ldots \ldots \ldots \ldots \ldots \ldots \ldots \ldots \ldots \ldots .43$

4.1.3 Esperança dos quadrados médios para as interações $\mathrm{E} \times \mathrm{N}^{\prime}, \mathrm{E} \times \mathrm{P}^{\prime}$

e $E \times K^{\prime}$

4.1.4 Esperança dos quadrados médios para $\mathrm{E} \times \mathrm{N}^{\prime} \times \mathrm{P}^{\prime}, \mathrm{E} \times \mathrm{N}^{\prime} \times \mathrm{K}^{\prime} \mathrm{e}$ $\mathrm{E} \times \mathrm{P}^{\prime} \times \mathrm{K}^{\prime}$ 50

4.2 Testes para homogeneidade de variâncias ................... 54

$4.2 .1 \quad$ Teste de Hartley . . . . . . . . . . . . . . . . . . . . . . . . . . . 54

4.2 .2 Teste de Bartlett . . . . . . . . . . . . . . . . . . . . . 55

4.3 Método de Cochran para ajuste dos graus de liberdade ............ 56

4.4 Análise de variância . . . . . . . . . . . . . . . . . . . . . . . 57

4.5 Teste de combinação de probabilidades . . . . . . . . . . . . . . . . . 64

4.6 Análise de variância ponderada . . . . . . . . . . . . . . . . . . . 68

5 CONCLUSÕES . . . . . . . . . . . . . . . . . . . . . . . . 71

REFERÊNCIAS BIBLIOGRÁFICAS . . . . . . . . . . . . . . . . . . 73

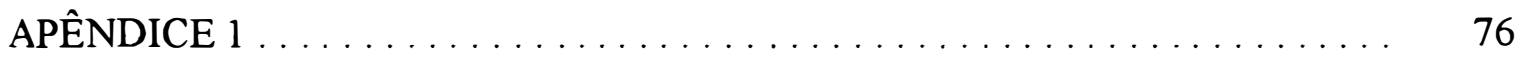

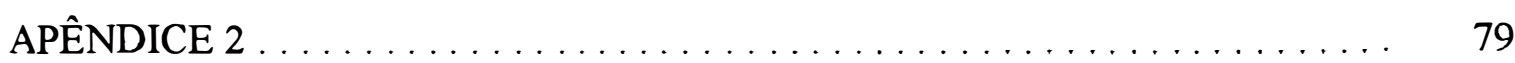

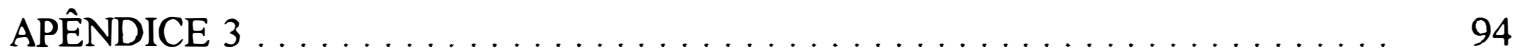




\section{LISTA DE TABELAS}

Página

1 Esquema de análise de variância para as análises individuais . . . . . . . . . . . . 19

2 Esquema de análise de variância para a análise conjunta. . . . . . . . . . . 21

3 Esperança dos quadrados médios para o modelo considerado na

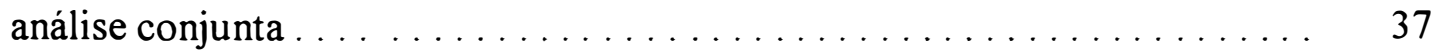

4 Quadrados médios dos resíduos para as análises individuais . . . . . . . . . 54

5 Esquema da análise de variância $\ldots \ldots \ldots \ldots \ldots \ldots \ldots \ldots \ldots \ldots$

6 Análise de variância conjunta . . . . . . . . . . . . . . . . . . . 58

$7 \quad$ Análise de variância utilizando o resíduo específico . . . . . . . . . . 62

8 Análise de variância não utilizando o resíduo específico . . . . . . . . . . 63

9 Teste de combinação de probabilidades para os contrastes de efeitos lineares . . . . . . . . . . . . . . . . . . . . . . 65

10 Teste de combinação de probabilidades para os contrastes de efeitos

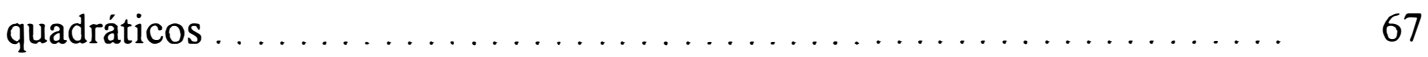

11 Análise de variância ponderada . . . . . . . . . . . . . . . . . . . . . 69

12 Produção de cana-planta $(\mathrm{t} / \mathrm{ha})$ em terra roxa legítima . . . . . . . . . . 77 


\title{
ANÁLISE DE GRUPOS DE EXPERIMENTOS COM HETEROGENEIDADE DE VARIÂNCIAS
}

\author{
Autor: WILSON ALVES DE OLIVEIRA \\ Orientadora: Prof ${ }^{\text {. }}$ Dr $^{\mathrm{a}}$. MARIA CRISTINA S. NOGUEIRA
}

\section{RESUMO}

$\mathrm{Na}$ análise de grupos de experimentos, alguns problemas como heterogeneidade de variâncias entre os diferentes experimentos, número de repetições diferentes, para tratamentos, em cada local, heterogeneidade das variâncias das interações e outros fatores podem ser encontrados.

Neste trabalho, para contornar o problema da heterogeneidade das variâncias das interações, é utilizada a técnica do resíduo específico, onde as esperanças dos quadrados médios, para os efeitos considerados na análise de variância, são demonstradas. Para o caso de heterogeneidade de variâncias entre os experimentos utilizou-se três métodos, o método de Cochran (1954), o teste de combinação de probabilidades e o método dos mínimos quadrados ponderados. Observou-se que o método dos mínimos quadrados ponderados é o mais indicado e que o resíduo específico pode ser usado, independentemente do método de análise utilizado, para o caso de heterogeneidade das variâncias das interações. 


\title{
THE ANALYSIS OF GROUPS OF EXPERIMENTS WITH HETEROGENEITY OF VARIANCES
}

\author{
Author: WILSON ALVES DE OLIVEIRA \\ Adviser: Prof ${ }^{a}$. $\mathrm{Dr}^{\mathrm{a}}$. MARIA CRISTINA S. NOGUEIRA
}

\section{SUMMARY}

The analysis of groups of experiments presents some problems as heterogeneity of variances from place to place, different number of replications to treatments in each place, heterogeneity of interactions variances and other factors.

This work presents the use of the specific error technic in order to solve the problem of heterogeneity of the interactions variances, where the expectations of the mean squares to the effects considered in the analysis of variance are shown. To solve the problem of heterogeneity of variances from place to place was used three methods, the Cochran method, the combining probabilities tests and the weighted least squares method. Was observed that the weighted least squares method is more indicated and that the specific error can be used, independently of method of analysis used to solve the problem of heterogeneity of the interactions variances. 


\section{INTRODUÇÃO}

Experimentos agrícolas envolvendo o mesmo fator ou grupos de fatores são geralmente conduzidos em vários locais distintos e/ou em várias ocasiões diferentes. Pode haver várias razões para isto. Algumas vezes, o objetivo da pesquisa é produzir recomendações que possam ser aplicadas a uma população que é extensiva, seja em espaço e/ou em tempo.

A análise estatística apropriada para os dados de uma série de experimentos irá variar com o objetivo da pesquisa. Todavia, os passos preliminares da análise tendem a ser os mesmos em todos os casos e uma aplicação, não crítica, dos métodos apropriados para análise conjunta, pode levar a conclusões errôneas.

A primeira crítica que surge, é que alguns componentes da soma de quadrados da interação de tratamentos com experimentos, podem ser muito maiores que outros, ou seja, a variância da interação não é constante.

Uma segunda crítica, é que as variâncias dos erros experimentais não são as mesmas em todos os experimentos. Em geral, esta hipótese será válida somente se todos experimentos foram conduzidos do mesmo modo, com a mesma quantidade de controle sobre as condições ambientais e com material da mesma variabilidade. Em experimentos com culturas este grau de uniformidade raramente é alcançado, porque a variabilidade natural entre áreas difere de um local para outro. Consequentemente, neste e em muitos outros tipos de experimentação cooperativa, espera-se a priori que as variâncias dos erros experimentais mudem de local para local.

Tomando como base o conjunto de dados a ser analisado e adotando o modelo misto, este estudo tem como objetivos: 
A obtenção das esperanças dos quadrados médios, na análise de variância, com a finalidade de obter o denominador apropriado para a aplicação do teste $\mathrm{F}$.

A decomposição da soma de quadrados dos efeitos de tratamentos e da soma de quadrados da interação de tratamentos com experimentos, em um conjunto de componentes ortogonais, visando contornar o problema da heterogeneidade de variâncias da interação, obtendo assim, o resíduo específico para cada um dos componentes.

Aplicação do método de Cochran (1954), do teste de combinação de probabilidades e do método dos mínimos quadrados ponderados, para contornar o problema da heterogeneidade de variâncias entre os experimentos. 


\section{REVISÃO DE LITERATURA}

Cochran (1937) considerou a análise estatística apropriada para experimentos repetidos em vários locais e em várias ocasiões diferentes, onde uma estimativa $x_{i}$ de um efeito de tratamento e uma estimativa $s_{i}$ do seu erro padrão, baseada em $n$ graus de liberdade é obtida. Segundo o autor, este tipo de dados pode surgir em muitos tipos de pesquisa, como por exemplo, séries de experimentos agrícolas, ou experimentos industriais sobre controle de qualidade. Os problemas considerados foram a estimação e teste de significância do efeito da média de tratamentos e sua variação de local para local, sendo estas as mais importantes questões preliminares em experimentos agrícolas deste tipo.

Ainda, segundo o autor, se as estimativas $x_{i}$, podem ser consideradas igualmente precisas, isto é, se as quantidades $s_{i}$ são todas estimativas do mesmo $\sigma$, a análise de variância é facilmente obtida. Quando isto não ocorre, a questão é mais dificil.

$\mathrm{Na}$ ausência de qualquer variação no efeito de tratamentos de local para local, a média ponderada, com ponderações $1 / s_{i}^{2}$, é sugerida como uma estimativa apropriada da média do efeito de tratamentos se pelo menos 15 graus de liberdade estão associados à estimativa $s_{i}$. Com menos de 15 graus de liberdade, segundo o autor, a média ponderada não é muito eficiente, e outras estimativas podem ser usadas. A estimativa de máxima verossimilhança é satisfatória do ponto de vista de informação, para valores de $n$ no mínimo iguais a 6 . A média não ponderada é particularmente satisfatória quando a precisão dos experimentos não varia muito e ainda, com baixos valores de $n$. Para valores de $n$ abaixo de 6 , a média ponderada com um limite superior escolhido arbitrariamente é a mais eficiente das três. A dificuldade está em obter o erro padrão para esta média. 
Yates \& Cochran (1938) sugerem um exame cuidadoso dos resultados experimentais antes de iniciar qualquer análise combinada, $\mathrm{e}$ indicam que $\mathrm{o}$ procedimento de análise de variância usual, apropriado para analisar os resultados de um só experimento, pode requerer modificação, devido à falta de igualdade nos erros dos diferentes experimentos, e devido à não homogeneidade dos componentes da interação de tratamentos com locais. Sugerem a partição em componentes apropriados das somas de quadrados devido a tratamentos e a tratamentos $\times$ locais.

$\mathrm{Se}$ os erros experimentais dos diferentes experimentos são substancialmente diferentes, o uso do teste $\mathrm{z}$ de Fisher junto com a estimativa do erro combinado pode levar a resultados enganosos. $\mathrm{Na}$ época deste artigo, a análise de variância era recente e era utilizado o teste $\mathrm{z}$, que é análogo ao teste $\mathrm{F}$ utilizado atualmente.

Os autores indicam que quando se têm experimentos quase discrepantes, "border-line", um teste de significância mais adequado é desejável. Uma forma de testar seria baseada na variância dos tratamentos observados, ou de alguma função análoga. Uma tal função, é a "soma dos quadrados dos desvios ponderados",

$$
Q=\sum_{i} w_{i}\left(x_{i}-\bar{x}_{w}\right)^{2}=\sum_{i} w_{i} x_{i}^{2}-\bar{x}_{w} \sum_{i} w_{i} x_{i}
$$

onde as ponderações $w_{i}$ são as recíprocas das estimativas das variâncias do resíduo, $\mathrm{e}$ $\bar{x}_{w}$ é a média ponderada, isto é,

$$
\bar{x}_{w}=\frac{\sum_{i} w_{i} x_{i}}{\sum_{i} w_{i}}
$$

Calcula-se, então,

$$
\chi^{\prime 2}=(k-1)+\sqrt{\frac{n-4}{n-1}\left\{\frac{n-2}{n} Q-(k-1)\right\}},
$$

onde, $k$ é o número de experimentos (estimativas) e $n$ é o número de graus de liberdade para cada estimativa; $\chi^{\prime 2}$ é distribuído aproximadamente como $\chi^{2}$ com $k$-1 graus de liberdade. Os autores alertam que este teste não deve ser usado quando $n$ for menor que 6. 
Se os experimentos são todos de mesma precisão, uma estimativa eficiente é a média não ponderada em cada experimento. Se, por outro lado, alguns dos experimentos são mais precisos que outros, a média não ponderada, por dar pesos iguais para ambos, pode fornecer uma estimativa menos precisa do que poderia ser obtida por processos estatísticos mais adequados.

Segundo os autores, a alternativa mais simples para a média não ponderada é a média ponderada, em que as ponderações são inversamente proporcionais às variâncias dos erros das estimativas obtidas nos vários experimentos. Esta média ponderada seria uma estimativa eficiente, se não houvesse variações na verdadeira resposta de experimento para experimento, e se além disso, as variâncias dos erros dos experimentos fossem exatamente conhecidas. Porém, se as variâncias dos erros são estimativas associadas a um pequeno número de graus de liberdade, a média ponderada perde bastante em eficiência e é frequentemente menos eficiente do que a média não ponderada.

Se a verdadeira resposta varia de experimento para experimento, tendo uma variância $\sigma_{0}^{2}$, e as variâncias dos erros são exatamente conhecidas, uma estimativa eficiente da resposta média é fornecida por uma média com ponderações inversamente proporcional a $\sigma_{0}^{2}+\sigma_{1}^{2}, \sigma_{0}^{2}+\sigma_{2}^{2}, \ldots$, onde $\sigma_{1}^{2}, \sigma_{2}^{2}, \ldots$ são as variâncias dos erros estimadas dos vários experimentos. Isto foi chamado de média semi-ponderada, já que as ponderações são intermediárias entre aquelas da média ponderada e as da média não ponderada.

Se a resposta não varia de experimento para experimento, mas as variâncias dos erros não são exatamente conhecidas, sendo estimadas com $n_{1}, n_{2}, \ldots$ graus de liberdade, uma estimativa eficiente é obtida pela solução da equação de máxima verossimilhança e é dada por:

$$
\mu=\frac{\sum_{i} \frac{\left(n_{i}+1\right) x_{i}}{n_{i} s_{i}^{2}+\left(x_{i}-\mu\right)^{2}}}{\sum_{i} \frac{\left(n_{i}+1\right)}{n_{i} s_{i}^{2}+\left(x_{i}-\mu\right)^{2}}} .
$$


Esta solução tem o efeito de atribuir baixas ponderações para os valores mais discrepantes do que seria dado pela média ponderada. Mas, este método não é normalmente justificável, exceto quando testes exatos de significância são requeridos e quando os $n_{i}$ são pequenos.

Assim, os métodos conhecidos para análise de variância ponderada, não são muito eficientes. Por outro lado, quando um conjunto de experimentos de precisão amplamente variada é encontrado, alguns métodos de desprezar os resultados dos experimentos menos precisos é necessário. O método mais simples seria rejeitar completamente os resultados dos experimentos menos precisos, mas isso envolve o esboço de uma linha arbitrária de divisão. É fácil ver que a inclusão ou exclusão de certos experimentos discrepantes, produz mudanças substanciais na resposta média.

Há um ponto adicional que deve ser examinado antes de usar qualquer forma de média em que as ponderações dependem da precisão relativa dos vários experimentos. Se a precisão está associada de qualquer modo com a magnitude da resposta, a média ponderada produzirá estimativas viciadas e não deve ser usada.

Para ver se existe associação deste tipo, os experimentos podem ser divididos em duas ou mais classes, de acordo com a precisão, e as diferenças entre a resposta média em cada classe examinada. Alternativamente, a regressão das respostas sobre o erro padrão dos experimentos pode ser calculada.

Welch (1951) modifica o $Q$ mencionado por Yates \& Cochran (1938), de forma que ele possa ser usado com a tabela F. O teste é dado da seguinte forma:

$$
v^{2}=\frac{\sum_{i} w_{i}\left(y_{i}-\hat{y}\right)^{2} /(k-1)}{\left[1+\frac{2(k-2)}{\left(k^{2}-1\right)} \sum_{i} \frac{1}{f_{i}}\left(1-\frac{w_{i}}{\sum_{i} w_{i}}\right)^{2}\right]},
$$

com números de graus de liberdades dados por: 


$$
\hat{f}_{1}=(k-1) \text { e } \hat{f}_{2}=\left[\frac{3}{\left(k^{2}-1\right)} \sum_{i} \frac{1}{f_{i}}\left(1-\frac{w_{i}}{\sum_{i} w_{i}}\right)^{2}\right]^{-1}
$$

onde, $w_{i}=\frac{n_{i}}{s_{i}^{2}}$, sendo $n_{i}$ o número de observações usadas para obter a variância estimada para tratamentos $\left(s_{i}^{2}\right) ; \hat{y}=\frac{\sum_{i} w_{i} y_{i}}{\sum_{i} w_{i}} ; f_{i}=n_{i}-1$ e $k=$ número de tratamentos.

Kempthorne (1952) considera que na análise conjunta, existem duas possiveis dificuldades para a interpretação da análise de variância. As dificuldades são:

1. Que $\sigma^{2}$ não é constante para os experimentos;

2. Que $\sigma_{t e}^{2}$ (componente de variância referente à interação de tratamentos com locais), depende da combinação de tratamentos e locais.

A suposição de que $\sigma^{2}$ é constante pode ser examinada pelo teste de Bartlett. É possivel que a variação em $\sigma^{2}$ seja resultado de não aditividade o que pode ser contornado por uma transformação dos dados, mas em geral, $\sigma^{2}$ irá variar de local para local, porque o material experimental não tem a mesma variabilidade.

O teste da interação de tratamentos com locais é complexo pela ocorrência de heterogeneidade da variância do erro.

Se há heterogeneidade da variância do erro, mas os componentes da interação $\sigma_{t e}^{2}$ podem ser considerados como homogêneos, ou ainda, se há diferentes números de repetições, um teste de significância aproximado para os efeitos de tratamentos sobre todos os locais, pode ser aplicado em uma análise de variância com as médias de tratamentos.

Meier (1953) considerou $x_{I}, \ldots, x_{k}$ sendo $k$ estimativas do parâmetro $\mu$, normal $\mathrm{e}$ independentemente distribuidas com média $\mu$ e variâncias $\sigma_{1}^{2}, \cdots, \sigma_{k}^{2}$. 
Considerou também, $s_{1}^{2}, \cdots, s_{k}^{2}$ estimativas independentes não viciadas de $\sigma_{i}^{2}$, cada uma com $n_{l}, \ldots, n_{k}$ graus de liberdade, respectivamente. $O$ autor comparou através de um exemplo, utilizando 4 experimentos, 3 métodos para combinar diversas estimativas de uma quantidade desconhecida. Os métodos utilizados foram:

(1) Assumir que todas as variâncias populacionais $\sigma_{i}^{2}$ são iguais, isto é, as 4 amostras são oriundas de uma única população (método de variâncias iguais).

(2) Considerar a possibilidade de variâncias populacionais diferentes, e usar as 4 variâncias estimadas, como se fossem as verdadeiras variâncias (variâncias desiguais, método não corrigido).

(3) Considerar a possibilidade de variâncias populacionais diferentes, mas fazer correções para permitir a variabilidade amostral das ponderações (variâncias desiguais, método corrigido).

Estas correções foram feitas da seguinte forma:

(a) Obtenção das variâncias estimadas $\left(s_{i}^{2}\right)$;

(b) Obtenção das ponderações, através da inversão dessas variâncias estimadas, isto é,

$$
w_{i}=\frac{1}{s_{1}^{2}}
$$

(c) Cálculo da média ponderada: $\bar{x}_{w}=\frac{\sum_{i} w_{l} x_{i}}{w}$, sendo $w=\sum_{i} w_{i}$;

(d) Cálculo da variância estimada de $\bar{x}_{w}$, dada pela fórmula:

$$
\operatorname{Var}\left(\bar{x}_{w}\right)=\frac{1}{w}\left[1+\frac{4}{w^{2}} \sum_{i} \frac{1}{n_{i}} w_{i}\left(w-w_{i}\right)\right]
$$

(e) Estimação dos graus de liberdade equivalentes, obtidos da seguinte forma:

$$
\hat{f}=\frac{w^{2}}{\sum_{i} \frac{w_{i}^{2}}{n_{i}}} .
$$


As conclusões que o autor tirou dos métodos comparados, neste exemplo, foram:

1. As estimativas de $\mu$ praticamente não diferiram, mas as variâncias estimadas diferiram substancialmente. Os métodos (1) e (3) ficaram bem próximos, diferindo por aproximadamente $2 \%$, mas o método (2) teve uma deficiência de $18 \%$ em relação ao método (3).

2. O exemplo ilustra o fato de que na análise para o caso de variâncias desiguais, o termo de correção requerido pode ser substancial. A quase igualdade dos resultados dos métodos (1) e (3) não foi surpresa, pelo fato de o teste de Bartlett não ter dado evidências contra a hipótese de igualdade de variâncias. $\mathrm{O}$ método (3), porém, é o procedimento mais conservativo e é aplicável sempre que a hipótese de variâncias iguais, é duvidosa, mesmo que o teste de homogeneidade de variâncias dê ou não, resultado significante.

Cochran \& Carroll (1953) consideram um número de estimativas $x_{i}$ $(i=1,2, \ldots, k)$, normal e independentemente distribuídas sobre a mesma média $\mu$ com diferentes variâncias $\sigma_{i}^{2}$. Se os valores de $\sigma_{i}^{2}$ são conhecidos, a melhor estimativa de $\mu$, em geral será a média ponderada

$$
\bar{x}_{w}=\frac{\sum_{i=1}^{k} w_{i} x_{i}}{w}, \text { onde } w_{i}=\frac{1}{\sigma_{i}^{2}} \text { e } w=\sum_{i} w_{i} .
$$

Se os $\sigma_{i}^{2}$ não são conhecidos, mas tem-se as variâncias estimadas $s_{i}^{2}$, com $\boldsymbol{n}_{i}$ graus de liberdade, respectivamente, neste caso, também é sugerido o uso de uma média ponderada com ponderações inversamente proporcionais às variâncias estimadas. Esta média é

$$
\bar{x}_{\hat{w}}=\frac{\sum_{i=1}^{k} \hat{w}_{i} x_{i}}{\hat{w}}, \text { onde } \hat{w}_{i}=\frac{1}{s_{i}^{2}} \text { e } \hat{w}=\sum_{i} \hat{w}_{i} .
$$

Os autores dizem que dados deste tipo podem ocorrer, por exemplo, quando $k$ laboratórios fazem determinações separadas $x_{i}$ de uma mesma quantidade 
física ou quimica, cada uma com um erro padrão estimado, ou quando está sendo feito um estudo dos resultados de $k$ experimentos repetidos, em que em cada um dos experimentos, a diferença $x_{i}$ entre um par específico de tratamentos foi observada.

Os autores apresentam alguns resultados sobre a distribuição de $\bar{x}_{\hat{w}}$. Entre eles, quando os graus de liberdade $n_{i}$ são todos iguais a $n$, a distribuição de $\bar{x}_{\hat{w}}$, quando o número de estimativas $k$ tende para infinito, é normal com média $\mu \mathrm{e}$ variância

$$
V\left(\bar{x}_{\hat{\psi}}\right)=\frac{(n-2)}{(n-4) w} .
$$

Segundo os autores, Cochran (1937) mostrou que uma estimativa não viciada da variância, quando os $n_{i}$ são iguais, é

$$
V\left(\bar{x}_{\hat{w}}\right)=\frac{n}{(n-4) \hat{w}} .
$$

Os autores fazem também, uma comparação com a média não ponderada. Dizem que uma alternativa simples para $\bar{x}_{\hat{w}}$ é a média não ponderada $\bar{x}$. A situação mais favorável para a média não ponderada é quando os $\sigma_{i}^{2}$ são todos iguais. Neste caso, a média não ponderada é completamente eficiente.

Os autores mostram em uma tabela, a precisão relativa da média ponderada para a não ponderada, quando $k=2$. Resumem esta tabela da seguinte forma: a média não ponderada é preferivel, se a razão da maior variância para a menor, não é maior que 2 . Se a razão encontra-se entre 2 e 3, a média ponderada é preferivel, quando os pesos são obtidos com pelo menos 12 graus de liberdade. Se a razão excede 3, a média ponderada é preferivel, mesmo se apenas 4 graus de liberdade estão associados à estimação dos pesos.

Cochran (1954) diz que o método mais simples de combinar estimativas obtidas em um número de diferentes experimentos é tomar a média aritmética das estimativas. Se no entanto, os experimentos variam no tamanho, ou parecem ser de diferentes precisões, o pesquisador pode surpreender-se se algum tipo de média 
ponderada for mais precisa. O autor fornece recomendações sobre os tipos de média ponderada que são apropriadas, as situações nas quais elas são apropriadas, e as circunstâncias nas quais a média não ponderada é preferida. Métodos para obter um desvio padrão vinculado à estimativa final, também são apresentados.

Neste trabalho, o autor utiliza a combinação de uma simples estimativa $x_{i}$, de cada experimento, considerando apenas 2 tratamentos. Quando cada experimento contém mais que 2 tratamentos, o autor indica uma análise combinada de todos os resultados experimentais. Sugere a utilização dos métodos propostos por Yates \& Cochran (1938), Cochran \& Cox (1950) e Kempthorne (1952), para contornar este problema.

$\mathrm{O}$ autor propõe um método que consiste em fazer um ajuste nos números de graus de liberdade, para a interação e para o resíduo médio, quando os Q.M. dos resíduos para cada experimento são muito diferentes. Os graus de liberdade para a interação podem ser tomados como:

$$
v_{1}=\frac{(k-1)^{2} V_{1}^{2}}{(k-2) V_{2}+V_{1}^{2}}
$$

onde,

$$
V_{\mathrm{I}}=\frac{\sum_{i} s_{i}^{2}}{k} ; \quad V_{2}=\frac{\sum_{i} s_{i}^{4}}{k}
$$

e $k$ é o número de experimentos ou locais.

Para o residuo médio, os graus de liberdade são obtidos por:

$$
v_{2}=\frac{\left(\sum_{i} s_{i}^{2}\right)^{2}}{\sum_{i} \frac{s_{i}^{4}}{n_{i}}} .
$$

Há, porém, a objeção adicional de que $F$ pode ser insensível em detectar a presença de interações, porque experimentos com baixa precisão recebem o mesmo peso como aqueles com alta precisão. 
Segundo o autor, um caminho alternativo, menos aberto para estas objeções, é basear o teste de interações na Soma de Quadrados dos desvios ponderados

$$
Q=\sum_{i=1}^{k} w_{i}\left(x_{i}-\bar{x}_{w}\right)^{2},
$$

onde,

$$
w_{i}=\frac{1}{s_{i}^{2}} \quad \mathrm{e} \quad \bar{x}_{w}=\frac{\sum_{i} w_{i} x_{i}}{\sum_{i} w_{i}} .
$$

Se o número de graus de liberdade $n_{i}$ for grande, Q segue a distribuição $\chi^{2}$ com $(\mathrm{k}-1)$ graus de liberdade. O autor faz uma adaptação ao método de Welch (1951), calculando uma quantidade auxiliar

$$
a=\sum_{i=1}^{k} \frac{1}{n_{i}}\left(1-\frac{w_{i}}{w}\right)^{2}, \quad \text { onde } \quad w=\sum_{i} w_{i}
$$

Então,

$$
F_{w}=\frac{Q}{(k-1)+\frac{2(k-2) a}{(k+1)}},
$$

com graus de liberdade dados por:

$$
v_{1}=(k-1) \text { e } \quad v_{2}=\frac{\left(k^{2}-1\right)}{3 a} .
$$

Para o autor, o teste de interações é importante, porque a presença de interações afeta a interpretação dos dados e pode determinar o tipo de média mais útil. Em um caso de extremo, o pesquisador deve levar em conta, a probabilidade de significância e qualquer outro conhecimento dos dados para decidir se considera interações como presente ou ausente. A decisão conservadora, quando em dúvida, é assumir interações presentes, já que as técnicas para esta situação permanecem válidas igualmente se interações estão ausentes. 
Cochran \& Cox (1957) dizem que na análise preliminar conjunta quando todos os experimentos têm o mesmo delineamento e o mesmo número de repetições, há dois elementos que podem ser utilizados para o denominador de $\mathrm{F}$ no teste para os efeitos médios de tratamentos, o QMTrat $\times$ Locais ou o QMResíduo Médio. Os autores mostram, utilizando as esperanças dos quadrados médios, que o denominador apropriado de F para este teste é o QMTrat $\times$ Locais.

Os autores fazem duas críticas sobre a análise preliminar.

A primeira crítica é que alguns componentes da S.Q.Trat. $\times$ Locais, podem ser muito maiores que outros, ou em termos matemáticos, que a variância da interação $\sigma_{\mu}^{2}$ não é constante. Isto ocorrerá, se o efeito de alguns tratamentos varia muito de local para local, enquanto que o dos outros varia menos ou não varia.

Se o quadrado médio da interação é heterogêneo, $\mathrm{o}$ teste $\mathrm{F}$ para tratamentos com interações é viciado, no sentido de que o valor de $\mathrm{F}$, obtido nas tabelas é muito baixo, isto é, obtêm-se muitos resultados significativos.

Segundo os autores, um melhor método de contornar esta dificuldade é decompor a soma de quadrados de tratamentos em um conjunto de componentes ortogonais, que fornecerá toda ou a maioria das informações de interesse. A S.Q.Interações é decomposta do mesmo modo, para se obter um resíduo específico, para testar cada um dos componentes. O único prejuízo para este procedimento é que os graus de liberdade no denominador de $\mathrm{F}$ ficam reduzidos.

Uma segunda crítica, afeta a hipótese de que as variâncias residuais $\sigma_{e}^{2}$ são as mesmas em todos experimentos. Em geral, esta hipótese será válida somente se todos experimentos foram conduzidos do mesmo modo, com a mesma quantidade de controle sobre condições ambientais e com material da mesma variabilidade.

A variação em $\sigma_{e}^{2}$ invalida o teste $\mathrm{F}$ para interações com o Q.M.Resíduo Médio. Embora o efeito sobre o nível de significância de $\mathrm{F}$ não seja conhecido exatamente, ele opera de forma que o uso do $\mathrm{F}$ tabelado produza muitos resultados significativos. 
Os autores apresentam um teste aproximado, que evita algumas das perdas em sensibilidade. Este teste difere do teste $F$, pois, fornece menos peso para experimentos que têm alta variância do residuo, e consequentemente, espera-se que seja mais sensível. Pode ser útil nos casos onde há duvida sobre o resultado dado pelo teste F e em casos onde uma análise mais eficiente é desejada.

Ainda, segundo os autores, para experimentos com diferentes números de repetições, a análise conjunta pode ser obtida, seguindo o procedimento padrão para dados desbalanceados.

Para esta situação, o Q.M.Resíduo Médio requer um pouco mais de atenção. Caso todos experimentos tenham a mesma variância do erro $\sigma_{e}^{2}$, o melhor procedimento consiste em ponderar cada Q.M.Resíduo, $s_{i}^{2}$, pelo número de graus de liberdade $n_{i}$. Mas se os experimentos variam na precisão, esta média ponderada é uma estimativa viciada do componente da variância do resíduo que entra no Q.M.Interação e no Q.M.Tratamentos. O componente correto é $\sum r_{i} \sigma_{i}^{2} / \sum r_{i}$ em vez de $\sum n_{i} \sigma_{i}^{2} / \sum n_{i}$. Consequentemente, a não ser que se esteja confiante que $\sigma_{e}^{2}$ é constante, é melhor ponderar os valores $s_{i}^{2}$ pelo número de repetições $\left(r_{i}\right)$.

$\mathrm{O}$ teste $\mathrm{F}$ para a interação é conduzido do mesmo modo como para experimentos balanceados, e está sujeito às críticas discutidas anteriormente. O teste $\mathrm{F}$ para tratamentos com o Q.M. de interações encontra uma nova dificuldade que é devido ao número desigual de repetições e está presente mesmo se todas as suposições requeridas para a análise de variância estão satisfeitas.

Pimentel Gomes (1987) apresenta uma aplicação do método da decomposição da interação de tratamentos com locais, em uma análise de um grupo de experimentos de adubação de algodão. $\mathrm{O}$ autor, ao fazer a análise conjunta dos diversos experimentos, considera individualmente os graus de liberdade relativos aos contrastes de tratamentos e estima cada contraste em cada local, obtendo assim, o quadrado médio apropriado para testar cada um dos contrastes. Porém, o autor alerta que este método tem 
a desvantagem de reduzir excessivamente o número de graus de liberdade para os testes, recomendando seu uso somente quando for grande o número de locais.

O autor, apresenta também, adaptação do método de Cochran (1954), para mais de 2 tratamentos.

Para testar a interação de tratamentos com experimentos, o número de graus de liberdade do numerador fica:

$$
n_{t e}=\frac{(t-1)(k-1)^{2} V_{1}^{2}}{(k-2) V_{2}+V_{1}^{2}},
$$

onde,

$$
V_{1}=\frac{\sum_{i} s_{i}^{2}}{k} ; \quad V_{2}=\frac{\sum_{i} s_{i}^{4}}{k}
$$

$k$ é o número de experimentos e $t$ é o número de tratamentos.

Para o denominador, o número de graus de liberdade fica:

$$
n_{r}=\frac{\left(\sum_{i} s_{i}^{2}\right)^{2}}{\sum_{i} \frac{s_{i}^{4}}{n_{i}}} .
$$

Fisher $^{1}$, citado por Conagin et al. (1993), desenvolveu o teste de combinação de probabilidades em grupos de experimentos. Este teste permite combinar a probabilidade dos resultados dos testes de comparação de médias ou testes de contrastes, independente do tipo de teste, experimento, número de repetições ou existência de interações.

Segundo os autores, foi demonstrado que toda função de distribuição contínua, que apresenta os primeiros momentos finitos, pode ser transformada em uma

\footnotetext{
${ }^{1}$ FISHER. R.A. Statistical methods for research workers. 4.ed. London: Oliver \& Boyd, 1932. 307p.
} 
distribuição uniforme no intervalo zero a um. Sendo $f(x)$ função densidade, definida em um intervalo $(\mathrm{a}, \mathrm{b})$ que pode ser de 0 a $\infty$ (distribuição $\chi^{2}$ ), ou de $-\infty$ a $+\infty$ (distribuição normal, ou det, etc.), pode ser transformada através de função $F(Z)$ definida como $\int_{-\infty}^{\infty} f(x) d x$ na função distribuição uniforme, definida no intervalo de zero a um, pelo resultado:

$$
\frac{\int_{-\infty}^{Z} f(x) d x}{\int_{-\infty}^{\infty} f(x) d x}=F(Z)=\operatorname{Pr}(0 \leq x \leq z) .
$$




\section{MATERIAL E MÉTODOS}

\subsection{Material}

Os dados analisados, referem-se a rendimentos de cana-de-açúcar, em t/ha, obtidos de um grupo de 11 experimentos instalados em terra roxa legítima, retirado de Malavolta et al. (1963).

Os experimentos foram instalados no esquema fatorial $3^{3}$, com confundimento de 2 graus de liberdade da interação tripla $\mathrm{N} \times \mathrm{P} \times \mathrm{K}$, de acordo com o grupo W (Yates, 1937).

O nitrogênio foi fornecido como salitre-do-Chile, nas doses de 0,60 e 120 $\mathrm{Kg} / \mathrm{ha}$, sendo $1 / 3$ no plantio, $1 / 3$ em cobertura, aos 3 meses, e $1 / 3$ aos 6 meses. $\mathrm{O}$ fósforo, usado nas doses de 0,75 e $150 \mathrm{Kg} / \mathrm{ha}$ de $\mathrm{P}_{2} \mathrm{O}_{5}$, foi fornecido todo no plantio, metade como superfosfato simples e metade como hiperfosfato. O potássio, aplicado sob a forma de cloreto, todo no plantio, teve as doses de 0,75 e $150 \mathrm{Kg} / \mathrm{ha} \mathrm{de} \mathrm{K}_{2} \mathrm{O}$.

Os autores constataram heterocedasticidade entre os experimentos (locais). Devido a esse fato, os 11 experimentos foram divididos em 2 subgrupos aproximadamente homocedásticos. Para o estudo em questão, foi considerado o conjunto dos 11 experimentos. 


\subsection{Métodos}

Para as análises individuais, o modelo matemático adotado foi o seguinte:

$$
y_{(i) j k l m}=\mu+b_{j}+N_{k}+P_{l}+K_{m}+(N P)_{k l}+(N K)_{k m}+(P K)_{l m}+e_{(i) j k l m}
$$

onde:

$y_{(i) j k l m}$ é a produção de cana-planta, em t/ha, observada no bloco $j$, para a $k l m$-ésima combinação dos fatores $N, P e K$ respectivamente, referente ao $\operatorname{local}(i) ; i=1, \ldots, I ; j=1, \ldots, J ; k=1, \ldots, K ; l=1, \ldots, L$ e $m=1, \ldots, M$;

$\mu \quad$ é a média geral;

$b_{j} \quad$ é o efeito dos blocos;

$N_{k} \quad$ é o efeito do fator Nitrogênio, considerado fixo;

$P_{l} \quad$ é o efeito do fator Fósforo, considerado fixo;

$K_{m} \quad$ é o efeito do fator Potássio, considerado fixo;

$(N P)_{k l} \quad$ é o efeito da interação Nitrogênio com Fósforo, considerado fixo;

$(N K)_{k m}$ é o efeito da interação Nitrogênio com Potássio, considerado fixo;

$(P K)_{l m}$ é o efeito da interação Fósforo com Potássio, considerado fixo;

$e_{(i) j k l m}$ é o erro experimental, correspondente às observações $y_{(i) j k l m}$, sendo $e_{(i) j k l m} \sim \operatorname{NID}\left(0, \sigma_{(i)}^{2}\right)$.

No caso dos experimentos fatoriais $3^{3}$ com confundimento de 2 graus de liberdade da interação tripla com blocos e com uma única repetição, segundo alguns autores, entre eles Banzatto e Kronka (1989), a parte não confundida da interação tripla, com 6 graus de liberdade funciona como resíduo. Visando obter melhores conclusões, pode-se desdobrar as interações duplas, isolando apenas a interação entre os componentes lineares, juntando os outros 3 componentes ao resíduo, para aumentar o seu número de graus de liberdade, que passará a 15 [ 6 da interação $\mathrm{N} \times \mathrm{P} \times \mathrm{K}$ (parte não confundida) e mais 3 de cada uma das interações duplas]. Assim, a análise de variância para as análises individuais seguiu o esquema dado na tabela 1. 
Tabela 1. Esquema de análise de variância para as análises individuais.

\begin{tabular}{lc}
\hline Causas de Variação & G.L. \\
\hline Blocos & 2 \\
N & 2 \\
$\mathrm{P}$ & 2 \\
$\mathrm{~K}$ & 2 \\
$\mathrm{~N}^{\prime} \times \mathrm{P}^{\prime}$ & 1 \\
$\mathrm{~N}^{\prime} \times \mathrm{K}^{\prime}$ & 1 \\
$\mathrm{P}^{\prime} \times \mathrm{K}^{\prime}$ & 1 \\
Resíduo & 15 \\
\hline TOTAL & 26
\end{tabular}

As análises individuais foram realizadas com a finalidade de obter os quadrados médios dos resíduos, para verificar a homocedasticidade entre os experimentos.

O modelo matemático adotado para a análise conjunta foi o seguinte:

$$
\begin{aligned}
y_{i j k l m} & =\mu+E_{i}+b_{(i) j}+N_{k}+P_{l}+K_{m}+(N P)_{k l}+(N K)_{k m}+(P K)_{l m}+(E N)_{i k}+(E P)_{i l}+ \\
& +(E K)_{i m}+(E N P)_{i k l}+(E N K)_{i k m}+(E P K)_{i l m}+e_{i j k l m}
\end{aligned}
$$

onde:

$y_{i j k l m}$ é a produção de cana-planta, em t/ha, observada no experimento $i$, bloco $j$, para a klm-ésima combinação dos fatores $N, P$ e $K$ respectivamente; $i=1, \ldots, \mathrm{I} ; j=1, \ldots, \mathrm{J} ; k=1, \ldots, \mathrm{K} ; l=1, \ldots, \mathrm{L}$ e $m=1, \ldots, \mathrm{M}$;

$\mu \quad$ é a média geral;

$E_{i} \quad$ é o efeito do experimento $i$, considerado aleatório;

$b_{(i) j} \quad$ é o efeito dos blocos dentro do experimento $i$, considerado fixo; 
$N_{k} \quad$ é o efeito do fator Nitrogênio, considerado fixo;

$P_{l} \quad$ é o efeito do fator Fósforo, considerado fixo;

$K_{m} \quad$ é o efeito do fator Potássio, considerado fixo;

$(N P)_{k l}$ é o efeito da interação Nitrogênio com Fósforo, considerado fixo;

$(N K)_{k m}$ é o efeito da interação Nitrogênio com Potássio, considerado fixo;

$(P K)_{l m} \quad$ é o efeito da interação Fósforo com Potássio, considerado fixo;

$(E N)_{i k} \quad$ é o efeito da interação Experimento com Nitrogênio, considerado aleatório;

$(E P)_{i l}$ é o efeito da interação Experimento com Fósforo, considerado aleatório;

$(E K)_{i m}$ é o efeito da interação Experimento com Potássio, considerado aleatório;

$(E N P)_{i k l}$ é o efeito da interação Experimento com $N e P$, considerado aleatório;

$(E N K)_{i k m}$ é o efeito da interação Experimento com $N e K$, considerado aleatório;

$(E P K)_{i l m}$ é o efeito da interação Experimento com $P$ e $K$, considerado aleatório;

$e_{i j k l m} \quad$ é o erro aleatório, normal e independentemente distribuído, com média zero e variância $\sigma^{2}$.

De acordo com o modelo, o esquema da análise de variância conjunta foi o considerado na tabela 2 . 
Tabela 2. Esquema de análise de variância para a análise conjunta.

\begin{tabular}{|c|c|}
\hline Causas de Variação & G.L. \\
\hline Experimentos (E) & 10 \\
\hline Blocos d. E & 22 \\
\hline $\mathrm{N}$ & 2 \\
\hline $\mathrm{P}$ & 2 \\
\hline $\mathrm{K}$ & 2 \\
\hline $\mathrm{N}^{\prime} \times \mathrm{P}^{\prime}$ & 1 \\
\hline$N^{\prime} \times K^{\prime}$ & 1 \\
\hline $\mathrm{P}^{\prime} \times \mathrm{K}^{\prime}$ & 1 \\
\hline $\mathrm{E} \times \mathrm{N}$ & 20 \\
\hline$E \times P$ & 20 \\
\hline $\mathrm{E} \times \mathrm{K}$ & 20 \\
\hline$E \times N^{\prime} \times P^{\prime}$ & 10 \\
\hline$E \times N^{\prime} \times K^{\prime}$ & 10 \\
\hline$E \times P^{\prime} \times K^{\prime}$ & 10 \\
\hline Resíduo Médio & 165 \\
\hline TOTAL & 296 \\
\hline
\end{tabular}

Quando se trabalha com grupos de experimentos, a primeira critica que surge é que alguns componentes da SQTratamentos $\times$ Locais podem ser muito maiores que outros, ou seja, a variância da interação não é constante. Isto ocorre pelo fato de que o efeito de alguns tratamentos varia muito de local para local.

Se o quadrado médio da interação é heterogêneo, o teste $F$ para tratamentos com interações é viciado, no sentido de que o valor de $\mathrm{F}$, obtido nas tabelas é muito baixo, isto é, obtendo-se muitos resultados significativos. 


\subsubsection{Testes para homogeneidade de variâncias}

Os testes para homogeneidade de variâncias mais comumente utilizados são os de Hartley e o de Bartlett.

\subsubsection{Teste de Hartley}

Milliken \& Johnson (1992) dizem que o teste de Hartley requer que todas as amostras sejam do mesmo tamanho e consiste em calcular o quociente

$$
F_{\max }=\frac{s_{\max }^{2}}{s_{\min }^{2}} .
$$

Rejeita-se a hipótese de igualdade de variâncias, se $F_{\max }>F_{\alpha, v, k}$, onde $v=n-1$, sendo $n$ o tamanho da amostra e $k$ o número de amostras.

\subsubsection{Teste de Bartlett}

Snedecor \& Cochran (1967) apresentam o teste de Bartlett da forma que se segue:

Se existem $k$ estimativas $s_{i}^{2}$, cada uma com o mesmo número de graus de liberdade $n$, o teste é

$$
M=2,3026 \cdot n\left(k \log \bar{s}^{2}-\sum_{i} \log s_{i}^{2}\right)
$$

onde,

$$
\bar{s}^{2}=\frac{\sum_{i} s_{i}^{2}}{k} .
$$

Sob a hipótese de nulidade de que cada $s_{i}^{2}$ é uma estimativa do mesmo $\sigma^{2}$, o quociente $M / C$ é distribuido aproximadamente como um $\chi^{2} \operatorname{com}(\mathrm{k}-1)$ graus de liberdade, onde, 


$$
C=1+\frac{k+1}{3 k n} \text {. }
$$

Se $\chi_{c}^{2}=\frac{M}{C}>\chi_{(k-1)}^{2}$, rejeita-se $H_{0}: \sigma_{1}^{2}=\sigma_{2}^{2}=\cdots=\sigma_{k}^{2}$.

No teste de Bartlett a estatística $M / C$ segue uma distribuição aproximada de $\chi^{2}$ somente quando os dados têm distribuição normal, sendo este teste muito sensível à falta de normalidade.

\subsubsection{Resíduo específico}

Um método proposto para contornar a heterogeneidade da variância da interação, é decompor a soma de quadrados de tratamentos em um conjunto de componentes ortogonais. A soma de quadrados da interação também é decomposta do mesmo modo, para se obter um resíduo específico, e testar cada um dos componentes com o componente obtido da interação de tratamentos com locais.

Como os níveis dos fatores de tratamentos, para o conjunto de dados considerado, são equidistantes, esta decomposição pode ser feita de modo simples, utilizando os coeficientes dos polinômios ortogonais.

O cálculo das somas de quadrados para a regressão linear e quadrática é feito da seguinte forma:

$$
\text { SQRLinear }=\frac{\left[\sum_{i=1}^{I}\left(\sum_{n=1}^{N} c_{1 n} T_{n}\right)\right]^{2}}{\operatorname{Ir} \sum c_{1 n}^{2}}
$$

e SQRQuadrática $=\frac{\left[\sum_{i=1}^{I}\left(\sum_{n=1}^{N} c_{2 n} T_{n}\right)\right]^{2}}{\operatorname{Ir} \sum c_{2 n}^{2}}$; 
onde, $c_{l n}$ e $c_{2 n}$ são os coeficientes dos polinômios ortogonais de $1^{\circ}$ e $2^{\circ}$ graus respectivamente;

$T_{n}$ é o total do $n$-ésimo nível do efeito considerado;

$r$ é o número de parcelas somadas para obter cada total $\left(T_{n}\right)$;

$N$ é o número de níveis do efeito considerado;

$I$ é o número de experimentos.

As somas de quadrados para as interações são dadas a seguir:

SQRLinear $\times$ Exper $=\frac{\sum_{i=1}^{I}\left(\sum_{n=1}^{N} c_{1 n} T_{n}\right)^{2}}{r \sum c_{1 n}^{2}}-S Q R L i n e a r$

e SQRQuadrática $\times$ Exper $=\frac{\sum_{i=1}^{I}\left(\sum_{n=1}^{N} c_{2 n} T_{n}\right)^{2}}{r \sum c_{2 n}^{2}}-S Q R Q u a d r a ́ t i c a$.

\subsubsection{Método de Cochran para ajuste dos graus de liberdade}

$\mathrm{Na}$ análise de grupos de experimentos, pode-se realizar a análise conjunta sem dificuldade, desde que o quociente entre o maior e o menor quadrado médio do resíduo das análises individuais, não seja muito grande, segundo alguns autores, maior do que 4 , ou se um teste para homogeneidade de variâncias não for significativo. Caso contrário, um método proposto por Cochran (1954), para contornar o problema de heterogeneidade de variâncias entre experimentos, consiste em fazer um ajuste nos números de graus de liberdade para a interação e para o resíduo médio.

Para testar a interação, experimentos com tratamentos, o número de graus de liberdade do numerador fica: 


$$
n_{E \times T}=\frac{(T-1)(I-1)^{2} V_{1}^{2}}{(I-2) V_{2}+V_{1}^{2}}
$$

onde,

$$
V_{1}=\frac{\sum_{i} s_{i}^{2}}{I} \quad ; \quad V_{2}=\frac{\sum_{i} s_{i}^{4}}{I} ;
$$

$I$ é o número de experimentos e $T$ é o número de tratamentos.

Para o denominador, o número de graus de liberdade fica:

$$
n_{r}=\frac{\left(\sum_{i} s_{i}^{2}\right)^{2}}{\sum_{i} \frac{s_{i}^{4}}{n_{i}}} .
$$

\subsubsection{Teste de combinação de probabilidades}

Segundo Fisher, citado por Conagin et al. (1993), o teste de combinação de probabilidades permite combinar a probabilidade dos resultados dos testes de comparação de médias ou testes de contrastes, independente do tipo de teste, experimento, número de repetições ou existência de interações.

\subsubsection{Combinação de resultados de testes individuais na análise de grupos de experimentos}

a) Se $X_{1}$ é uma variável aleatória distribuída normalmente com média $\mu_{1}$ e variância $\sigma_{i}^{2}$, então:

$$
Z=\frac{X_{1}-\mu_{1}}{\sigma_{i}}
$$

é uma variável aleatória, distribuída normalmente com média zero e variância um. 
b) $\operatorname{Se} X_{1}, X_{2}, \ldots, X_{\mathrm{n}}$ são variáveis aleatórias $\sim N\left(\mu_{i}, \sigma_{i}^{2}\right)$, então

$$
Z=\frac{\bar{X}_{i}-\mu_{i}}{\sigma_{\bar{X}_{i}}}=\frac{\sqrt{n}\left(\bar{X}_{i}-\mu_{i}\right)}{\sigma_{i}} \mathrm{e} \sim \mathrm{N}(0,1) .
$$

c) $\operatorname{Se} X_{11}, X_{12}, \ldots, X_{1 n_{1}}$ e $X_{21}, X_{22}, \ldots, X_{2 n_{2}}$ são variáveis aleatórias, então

$$
Z_{1}=\frac{\sqrt{n_{1}}\left(\bar{X}_{1}-\mu_{1}\right)}{\sigma_{1}} \text { e } Z_{2}=\frac{\sqrt{n_{2}}\left(\bar{X}_{2}-\mu_{2}\right)}{\sigma_{2}} \text { são } \sim \mathrm{N}(0,1) \text {. }
$$

Se $\sigma_{1}=\sigma_{2}=\sigma$ (homocedasticidade), então

$$
Z=\frac{\left(\bar{X}_{1}-\bar{X}_{2}\right)-\left(\mu_{1}-\mu_{2}\right)}{\sigma_{\bar{X}_{1}-\bar{X}_{2}}}=\frac{\left(\bar{X}_{1}-\bar{X}_{2}\right)-\left(\mu_{1}-\mu_{2}\right)}{\sigma \sqrt{\frac{1}{n_{1}}+\frac{1}{n_{2}}}} \text { tem } \sim \mathrm{N}(0,1) .
$$

Sob $H_{0}: \mu_{1}=\mu_{2}=\mu$, então

$$
Z=\frac{\left(\bar{X}_{1}-\bar{X}_{2}\right)}{\sigma \sqrt{\frac{1}{n_{1}}+\frac{1}{n_{2}}}} \mathrm{e} \sim \mathrm{N}(0,1)
$$

A função densidade será $f(Z)$ normal com média zero e variância um. A função cumulativa $F(Z)$ tem distribuição uniforme variando de zero a um.

\subsubsection{Teorema da combinação de probabilidades}

Se um experimento produz um teste $Z_{1}$ com probabilidade $F\left(Z_{1}\right)$ e outro experimento, montado independentemente do primeiro, produz um teste $Z_{2}$ com probabilidade $F\left(Z_{2}\right)$, a probabilidade de se obter um resultado conjunto $Z_{1}$ e $Z_{2}$ é dada por $\mathrm{P}\left(Z_{1}, Z_{2}\right)=\mathrm{F}\left(Z_{1}\right) \cdot \mathrm{F}\left(Z_{2}\right)=\mathrm{P}\left(Z_{1}\right) \cdot \mathrm{P}\left(Z_{2}\right)$, onde $Z_{1} \sim \mathrm{N}(0,1), Z_{2} \sim \mathrm{N}(0,1)$ e $\mathrm{F}\left(Z_{1}\right)$, $\mathrm{F}\left(\mathrm{Z}_{2}\right)$ têm distribuição uniforme no intervalo de zero a um.

Os resultados de quaisquer pares de testes são uniformemente distribuídos sobre um quadrado no primeiro quadrante, cada lado variando de zero a um. Uma hipérbole retangular $\lambda=F\left(Z_{1}\right) \cdot F\left(Z_{2}\right)$ define o lugar geométrico de todos os pares de 
testes que apresentam igual verossimilhança $\lambda$. A significância dos resultados combinados é dada por $P$, onde:

$$
\begin{aligned}
P & =1-\int_{\lambda}^{1} \int_{F\left(Z_{2}\right)}^{1} d F\left(Z_{1}\right) d F\left(Z_{2}\right)=1-\int_{\lambda}^{1} d F\left(Z_{1}\right)\left[F\left(Z_{2}\right)\right]_{\lambda / F\left(z_{1}\right)}^{1}= \\
& =1-\int_{\lambda}^{1} d F\left(Z_{1}\right)\left[1-\frac{\lambda}{F\left(Z_{1}\right)}\right]=1-\int_{\lambda}^{1} d F\left(Z_{1}\right)+\int_{\lambda}^{1} \frac{\lambda}{F\left(Z_{1}\right)} d F\left(Z_{1}\right)= \\
& =1-\left[F\left(Z_{1}\right)\right]_{\lambda}^{1}+\lambda \ln \left[F\left(Z_{1}\right)\right]_{\lambda}^{1}=1-(1-\lambda)+\lambda \ln (1-\lambda)=\lambda+\lambda \ln (1)-\lambda \ln (\lambda)
\end{aligned}
$$

logo,

$$
P=\lambda[1-\ln (\lambda)]
$$

No caso da combinação de probabilidade de três experimentos, tem-se:

$$
P=\lambda\left\{1-\ln (\lambda)+\frac{[\ln (\lambda)]^{2}}{2}\right\}
$$

$\mathrm{Na}$ combinação de probabilidade dos testes de $\mathrm{n}$ experimentos, tem-se:

$$
P=\lambda\left\{1-\ln (\lambda)+\frac{[\ln (\lambda)]^{2}}{2 !}-\frac{[\ln (\lambda)]^{3}}{3 !}+\cdots+(-1)^{n-1} \frac{[\ln (\lambda)]^{n-1}}{(n-1) !}\right\},
$$

onde:

$$
\lambda=F\left(Z_{1}\right) \cdot F\left(Z_{2}\right) \ldots . F\left(Z_{n}\right)
$$

A equação que fornece a probabilidade $P$, apresenta uma relação muito estreita com a distribuição de $\chi^{2} \operatorname{com} 2 \mathrm{n}$ graus de liberdade, o que possibilita usar a tabela desta distribuição com $2 \mathrm{n}$ graus de liberdade, para a avaliação da significância dos resultados. A função densidade de $\chi^{2} \operatorname{com} 2 \mathrm{n}$ graus de liberdade é dada por:

$$
g\left(\chi^{2}\right)=\frac{1}{2^{n} \Gamma(n)}\left(\chi^{2}\right)^{n-1} e^{\chi^{2}-2} \quad(0, \infty)
$$


Prova-se que:

$$
\int_{A}^{\infty} g\left(\chi^{2}\right) d \chi^{2}=e^{-A i 2}\left[1+\frac{A}{2}+\frac{(A 2)^{2}}{2 !}+\cdots+\frac{(A / 2)^{n-1}}{(n-1) !}\right] .
$$

Considerando: $\lambda=e^{-A / 2}$, tem-se que: $\ln \lambda=-A / 2$ portanto,

$$
\begin{aligned}
& A=-2 \ln (\lambda) . \\
& \int_{A}^{\infty} g\left(\chi^{2}\right) d \chi^{2}=\int_{-2 \ln (\lambda)}^{\infty} g\left(\chi^{2}\right) d \chi^{2}= \\
& =\lambda\left\{1-\ln (\lambda)+\frac{[\ln (\lambda)]^{2}}{2 !}-\frac{[\ln (\lambda)]^{3}}{3 !}+\cdots+(-1)^{n-1} \frac{[\ln (\lambda)]^{n-1}}{(n-1) !}\right\} \\
& \mathrm{e} \quad-2 \ln (\lambda)=-2 \ln \left[F\left(Z_{1}\right) \cdot F\left(Z_{2}\right) \ldots . F\left(Z_{n}\right)\right]=-2 \sum_{i=1}^{n} \ln \left[F\left(Z_{i}\right)\right] .
\end{aligned}
$$

Como $-2 \sum_{i=1}^{n} \ln \left[F\left(Z_{i}\right)\right]$ é um $\chi_{2 n}^{2}$ face à aditividade do $\chi^{2}$, conclui-se que:

$-2 \ln F\left(Z_{i}\right) \quad$ é $\quad \chi_{(2)}^{2}$, isto é, $-2 \ln \left[F\left(Z_{i}\right)\right]$ é distribuído como $\chi^{2}$ com 2 graus de liberdade. Aqui, $Z_{i}$ é o $t_{i}$ de Student, então, calcula-se

$$
t_{i}=\frac{\hat{Y}}{s(\hat{Y})}
$$

onde, $\hat{Y}$ é a estimativa de um contraste de interesse e $s(\hat{Y})$ é a estimativa do desvio padrão do contraste. Obtido $t_{i}$, calcula-se a $\mathrm{P}\left(t_{i}\right)$ e, a seguir $-2 \ln \left[P\left(t_{i}\right)\right]$, combinando-se os resultados dos experimentos regionais, isto é, calculando-se $\chi_{2 n}^{2}=-2 \sum_{i=1}^{n} \ln \left[P\left(t_{i}\right)\right]$.

Se $\chi^{2}$ obtido for maior que $\chi_{(2 n)}^{2}$ a $5 \%$ ou $1 \%$, o contraste que se está testando foi significativo na região considerada.

$\mathrm{O}$ teste $t_{i}$ é unilateral, isto é, $\mathrm{P}\left(t_{i}\right)$ avalia sempre a cauda à direita de $\mathrm{f}(t)$.

Neste tipo de teste, as interações experimentos com tratamentos não precisam ser levadas em consideração. Se o valor de $\chi_{(2 n)}^{2}$ for significativo, é que na grande maioria dos locais se verificou uma certa superioridade do contraste e, no 
conjunto esta superioridade se revelou significativa. Se houver muitos resultados discordantes, o $\chi_{(2 n)}^{2}$ global não será significativo.

\subsubsection{Análise de variância ponderada}

Quando existe heterocedasticidade entre os experimentos, segundo Cochran (1957), o teste $F$ de interações com o resíduo médio, não é válido. Embora o efeito sobre o nível de significância de F não seja conhecido exatamente, ele opera de forma que se tenham muitos resultados significativos. A alternativa mais simples para isso, é o uso da análise de variância ponderada.

Quando se usa a análise ponderada, a soma de quadrados dos resíduos ponderados

$$
\sum_{i} w_{i}\left(x_{i}-\bar{x}_{w}\right)^{2}
$$

é minimizada, sendo $w_{i}=1 / s_{i}^{2}, s_{i}^{2}$ a variância estimada por experimento, $x_{i}$ o valor observado da variável resposta e $\bar{x}_{w}$ a média ponderada dada por:

$$
\bar{x}_{w}=\frac{\sum_{i=1}^{I} w_{i} x_{i}}{w}
$$

onde, $w=\sum w_{i}$ e $I$ é o número de experimentos.

O uso da ponderação $r_{i} / s_{i}^{2}$, onde $r_{i}$ é o número de repetições por experimento, é melhor que a ponderação $1 / s_{i}^{2}$, quando se têm diferentes números de repetições para os experimentos.

As equações normais usadas na análise ponderada são: $\hat{\beta}=\left(X^{\prime} W X\right)^{-} X^{\prime} W Y$, onde $W$ é uma matriz diagonal contendo os valores das ponderações $w_{i}$.

Se as ponderações são proporcionais aos recíprocos das variâncias dos resíduos, então, as estimativas de mínimos quadrados ponderados são BLUE (Best Linear Unbiased Estimators). 


\section{RESULTADOS E DISCUSSÃO}

\subsection{Esperança dos quadrados médios}

Para compor o teste $\mathrm{F}$, é necessário estudar a natureza dos quadrados médios na análise de variância.

Para o modelo

$$
\begin{aligned}
& y_{i j k l m}=\mu+E_{i}+b_{(i) j}+N_{k}+P_{l}+K_{m}+(N P)_{k l}+(N K)_{k m}+(P K)_{l m}+ \\
& +(E N)_{i k}+(E P)_{i l}+(E K)_{i m}+(E N P)_{i k l}+(E N K)_{i k m}+(E P K)_{i l m}+e_{i j k l m},
\end{aligned}
$$

onde:

$y_{i j k l m} \quad$ é a produção de cana-planta, em $\mathrm{t} / \mathrm{h}$, observada no experimento $i$, bloco $j$, para a $\mathrm{klm}$-ésima combinação dos fatores $N, P$ e $K$ respectivamente; $i=1, \ldots, I ; j=1, \ldots, J ; k=1, \ldots, K ; l=1, \ldots, L$ e $m=1, \ldots, M$

$\mu \quad$ é a média geral;

$E_{i} \quad$ é o efeito do experimento $i$, considerado aleatório;

$b_{(i) j} \quad$ é o efeito dos blocos dentro do experimento $i$, considerado fixo;

$N_{k} \quad$ é o efeito do fator Nitrogênio, considerado fixo;

$P_{l} \quad$ é o efeito do fator Fósforo, considerado fixo;

$K_{m} \quad$ é o efeito do fator Potássio, considerado fixo;

$(N P)_{k l}$ é o efeito da interação Nitrogênio com Fósforo, considerado fixo;

$(N K)_{k m}$ é o efeito da interação Nitrogênio com Potássio, considerado fixo;

$(P K)_{l m}$ é o efeito da interação Fósforo com Potássio, considerado fixo;

$(E N)_{i k}$ é o efeito da interação Experimento com Nitrogênio, considerado aleatório; 
$(E P)_{i l}$ é o efeito da interação Experimento com Fósforo, considerado aleatório; $(E K)_{i m}$ é o efeito da interação Experimento com Potássio, considerado aleatório; $(E N P)_{i k l}$ é o efeito da interação Experimento com $N e P$, considerado aleatório; $(E N K)_{i k m}$ é o efeito da interação Experimento com $N e K$, considerado aleatório; $(E P K)_{i l m}$ é o efeito da interação Experimento com $P$ e $K$, considerado aleatório; $e_{i j k l m} \quad$ é o erro aleatório, normal e independentemente distribuído, com média zero e variância $\sigma^{2}$.

com as seguintes restrições aplicadas:

$$
\begin{array}{llll}
\sum_{i} b_{(i) j}=0, \text { para todo } j ; & \sum_{j} b_{(i) j}=0, \text { para todo } i ; & \Rightarrow & \sum_{i, j} b_{(i) j}=0 ; \\
\sum_{k} N_{k}=0 ; & \sum_{l} P_{l}=0 ; & & \sum_{m} K_{m}=0 ; \\
\sum_{k}(N P)_{k l}=0, \text { para todo } l ; & \sum_{l}(N P)_{k l}=0, \text { para todo } k \Rightarrow & \sum_{k, l}(N P)_{k l}=0 ; \\
\sum_{k}(N K)_{k m}=0, \text { para todo } m ; & \sum_{m}(N K)_{k m}=0, \text { para todo } k \Rightarrow & \sum_{k, m}(N K)_{k m}=0 ; \\
\sum_{l}(P K)_{l m}=0, \text { para todo } m ; & \sum_{m}(P K)_{l m}=0, \text { para todo } l \Rightarrow \sum_{l . m}(P K)_{l m}=0 ;
\end{array}
$$

e ainda, que:

$E_{i}$ é normal e independentemente distribuído, com média zero e variância $\sigma_{E}^{2}$, isto é, $E_{i}: \operatorname{NID}\left(0, \sigma_{E}^{2}\right)$;
$b_{(i) j}:$
$E\left(b_{(i) j}\right)=b_{(i) j}$
$E\left(b_{(i) j}^{2}\right)=b_{(i) j}^{2} ;$
$N_{k}: \quad E\left(N_{k}\right)=N_{k}$
$E\left(N_{k}^{2}\right)=N_{k}^{2}$
$P_{l}: \quad E\left(P_{l}\right)=P_{l}$
$E\left(P_{l}^{2}\right)=P_{l}^{2}$;
$K_{m}:$
$E\left(K_{m}\right)=K_{m}$
$E\left(K_{m}^{2}\right)=K_{m}^{2}$; 


$$
\begin{array}{lll}
(N P)_{k l}: & E\left[(N P)_{k l}\right]=(N P)_{k l} & E\left[(N P)_{k l}^{2}\right]=(N P)_{k l}^{2} ; \\
(N K)_{k m}: & E\left[(N K)_{k m}\right]=(N K)_{k m} & E\left[(N K)_{k m}^{2}\right]=(N K)_{k m}^{2} ; \\
(P K)_{l m}: & E\left[(P K)_{l m}\right]=(P K)_{l m} & E\left[(P K)_{l m}^{2}\right]=(P K)_{l m}^{2} ; \\
(E N)_{i k}: & N I D\left(0, \sigma_{E N}^{2}\right) ; \\
(E P)_{i l}: & N I D\left(0, \sigma_{E P}^{2}\right) ; \\
(E K)_{i m}: & N I D\left(0, \sigma_{E K}^{2}\right) ; \\
(E N P)_{i k l}: & N I D\left(0, \sigma_{E N P}^{2}\right) ; \\
(E N K)_{i k m}: & N I D\left(0, \sigma_{E N K}^{2}\right) ; \\
(E P K)_{i l m}: & N I D\left(0, \sigma_{E P K}^{2}\right) ; \\
e_{i j k l m:} & N I D\left(0, \sigma^{2}\right) .
\end{array}
$$

Além disso, os efeitos aleatórios do modelo não são correlacionados.

A esperança do quadrado médio para o efeito de $\mathrm{N}$ é demonstrada a seguir:

$$
E(Q M N)=E\left(\frac{S Q N}{K-1}\right)=\frac{1}{K-1} E(S Q N)
$$

onde, a soma de quadrados para o efeito do Nitrogênio é dada pela seguinte expressão:

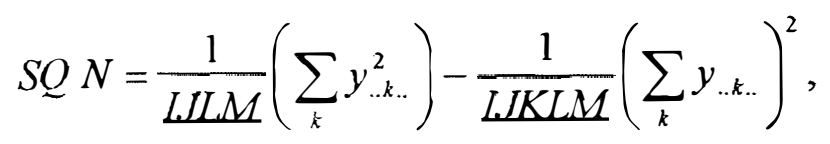

assim,

$$
E(S Q N)=\frac{1}{L U L M} E\left(\sum_{k} y_{. . . . .}^{2}\right)-\frac{1}{\underline{L K L L M}} E\left(\sum_{k} y_{\ldots . . .}\right)^{2}
$$


Da expressão (1), tem-se:

(i) $\quad \sum_{k} y_{. . k . .}^{2}=\sum_{k}\left(\sum_{i j l m} y_{i j k l m}\right)^{2}=$

$=\sum_{k}\left\{\sum_{i j l m}\left(\begin{array}{l}\mu+E_{i}+b_{(i) j}+N_{k}+P_{l}+K_{m}+(N P)_{k l}+(N K)_{k m}+(P K)_{l m}+(E N)_{i k}+ \\ +(E P)_{i l}+(E K)_{i m}+(E N P)_{i k l}+(E N K)_{i k m}+(E P K)_{i l m}+e_{i j k l m}\end{array}\right)\right\}^{2}=$

$=\sum_{k}\left[I J L M \mu+J L M \sum_{i} E_{i}+L M \sum_{i j} b_{(i) j}+I J L M N_{k}+I J M \sum_{l} P_{l}+I J L \sum_{m} K_{m}+\right.$

$+I J M \sum_{l}(N P)_{k l}+I J L \sum_{m}(N K)_{k m}+I J \sum_{l m}(P K)_{l m}+J L M \sum_{i}(E N)_{i k}+J M \sum_{i l}(E P)_{i l}+$

$\left.+J L \sum_{i m}(E K)_{i m}+J M \sum_{i l}(E N P)_{i k l}+J L \sum_{i m}(E N K)_{i l m}+J \sum_{i l m}(E P K)_{i l m}+\sum_{i j l m} e_{i j k l m}\right]^{2}=$

$=\sum_{k}\left\{I^{2} J^{2} L^{2} M^{2} \mu^{2}+J^{2} L^{2} M^{2}\left(\sum_{i} E_{i}\right)^{2}+I^{2} J^{2} L^{2} M^{2} N_{k}^{2}+J^{2} L^{2} M^{2}\left[\sum_{i}(E N)_{i k}\right]^{2}+\right.$

$+J^{2} M^{2}\left[\sum_{i l}(E P)_{i l}\right]^{2}+J^{2} L^{2}\left[\sum_{i m}(E X)_{i m}\right]^{2}+J^{2} M^{2}\left[\sum_{i l}(E N P)_{i k l}\right]^{2}+$

$\left.+J^{2} L^{2}\left[\sum_{i m}(E N K)_{i k m}\right]^{2}+J^{2}\left[\sum_{i l m}(E P K)_{i l m}\right]^{2}+\left(\sum_{i j l m} e_{i j k l m}\right)^{2}+2(I J L M \mu)\left(I J L M N_{k}\right)+d p\right\}$

onde, $d p$ refere-se aos duplos produtos que envolvem pelo menos um efeito aleatório, que são nulos quando aplica-se esperança matemática nos mesmos. Assim: 


$$
\begin{aligned}
& \sum_{k} y_{. . . . .}^{2}=K I^{2} J^{2} L^{2} M^{2} \mu^{2}+K J^{2} L^{2} M^{2}\left(\sum_{i} E_{i}\right)^{2}+I^{2} J^{2} L^{2} M^{2} \sum_{k} N_{k}^{2}+ \\
& +J^{2} L^{2} M^{2} \sum_{k}\left[\sum_{i}(E N)_{i k}\right]^{2}+K J^{2} M^{2}\left[\sum_{i l}(E P)_{i l}\right]^{2}+K J^{2} L^{2}\left[\sum_{i m}(E K)_{i m}\right]^{2}+ \\
& +J^{2} M^{2} \sum_{k}\left[\sum_{l l}(E N P)_{i k l}\right]^{2}+J^{2} L^{2} \sum_{k}\left[\sum_{l m}(E N K)_{i k m}\right]^{2}+K J^{2}\left[\sum_{l l m}(E P K)_{i l m}\right]^{2}+ \\
& +\sum_{k}\left(\sum_{i j l m} e_{i j k l m}\right)^{2}+2 I^{2} J^{2} L^{2} M^{2} \mu \underbrace{\sum_{k} N_{k}}_{0}+d p
\end{aligned}
$$

então,

$$
\begin{aligned}
& E\left(\sum_{k} y_{. . k . .}^{2}\right)=E\left(K I^{2} J^{2} L^{2} M^{2} \mu^{2}\right)+E\left[K J^{2} L^{2} M^{2}\left(\sum_{i} E_{i}\right)^{2}\right]+E\left[I^{2} J^{2} L^{2} M^{2} \sum_{k} N_{k}^{2}\right]+ \\
& +E\left\{J^{2} L^{2} M^{2} \sum_{k}\left[\sum_{i}(E N)_{i k}\right]^{2}\right\}+E\left\{K J^{2} M^{2}\left[\sum_{i l}(E P)_{i l}\right]^{2}\right\}+E\left\{K J^{2} L^{2}\left[\sum_{i m}(E K)_{i m}\right]^{2}\right\}+ \\
& +E\left\{J^{2} M^{2} \sum_{k}\left[\sum_{i l}(E N P)_{i k l}\right]^{2}\right\}+E\left\{J^{2} L^{2} \sum_{k}\left[\sum_{i m}(E N K)_{i k m}\right]^{2}\right\}+ \\
& +E\left\{K J^{2}\left[\sum_{i l m}(E P K)_{i l m}\right]^{2}\right\}+E\left[\sum_{k}\left(\sum_{i j l m} e_{i j k l m}\right)^{2}\right]= \\
& =K I^{2} J^{2} L^{2} M^{2} \mu^{2}+K J^{2} L^{2} M^{2} I \sigma_{E}^{2}+I^{2} J^{2} L^{2} M^{2} \sum_{k} N_{k}^{2}+J^{2} L^{2} M^{2} I K \sigma_{E N}^{2}+ \\
& +K J^{2} M^{2} I L \sigma_{E P}^{2}+K J^{2} L^{2} I M \sigma_{E K}^{2}+J^{2} M^{2} I K L \sigma_{E N P}^{2}+ \\
& +J^{2} L^{2} I K M \sigma_{E N K}^{2}+K J^{2} I L M \sigma_{E P K}^{2}+K I J M \sigma^{2}
\end{aligned}
$$

pois,

$$
\begin{aligned}
& E\left(\sum_{i} E_{i}\right)^{2}=E\left(E_{1}+E_{2}+\cdots+E_{l}\right)^{2}=E\left(E_{1}^{2}+E_{2}^{2}+\cdots+E_{I}^{2}+2 E_{1} E_{2}+\cdots+2 E_{l-1} E_{I}\right)= \\
& =\sigma_{E}^{2}+\sigma_{E}^{2}+\cdots+\sigma_{E}^{2}+0+\cdots+0=I \sigma_{E}^{2}
\end{aligned}
$$




$$
\begin{aligned}
& E\left\{\sum_{k}\left[\sum_{i}(E N)_{i k}\right]^{2}\right\}=E\left\{\sum_{k}\left[(E N)_{1 k}+\cdots+(E N)_{I k}\right]^{2}\right\}= \\
& =E\left\{\sum_{k}\left[(E N)_{1 k}^{2}+\cdots+(E N)_{I k}^{2}+d p\right]\right\}= \\
& =E\left\{(E N)_{11}^{2}+\cdots+(E N)_{1 K}^{2}+\cdots+(E N)_{I 1}^{2}+\cdots+(E N)_{I K}^{2}\right\}= \\
& =\sigma_{E N}^{2}+\cdots+\sigma_{E N}^{2}+\cdots+\sigma_{E N}^{2}+\cdots+\sigma_{E N}^{2}=I K \sigma_{E N}^{2},
\end{aligned}
$$

de modo análogo obtêm-se as expressões restantes.

Logo,

$$
\begin{aligned}
& \frac{1}{\underline{I J L M}} E\left(\sum_{k} y_{. . . .}^{2}\right)=I J K L M \mu^{2}+J K L M \sigma_{E}^{2}+I J L M \sum_{k} N_{k}^{2}+J K L M \sigma_{E N}^{2}+ \\
& +J K M \sigma_{E P}^{2}+J K L \sigma_{E K}^{2}+J K M \sigma_{E N P}^{2}+J K L \sigma_{E N K}^{2}+J K \sigma_{E P K}^{2}+K \sigma^{2} . \\
& \text { (ii) }\left(\sum_{k} y_{. . k . .}\right)^{2}=\left[\sum_{k}\left(\sum_{i j l m} y_{i j k l m}\right)\right]^{2}= \\
& =\left\{\sum_{k}\left[\sum_{i j l m}\left(\begin{array}{l}
\left.\mu+E_{i}+b_{(i),}+N_{k}+P_{l}+K_{m}+(N P)_{k l}+(N K)_{k m}+(P K)_{l m}+(E N)_{i k}+\right) \\
+(E P)_{i l}+(E K)_{i m}+(E N P)_{i k l}+(E N K)_{i k m}+(E P K)_{i l m}+e_{i j k l m}
\end{array}\right]\right\}^{2}=\right. \\
& =\left[I J K L M \mu+J K L M \sum_{i} E_{i}+K L M \sum_{i j} b_{(i) \jmath}+I J L M \sum_{k} N_{k}+I J K M \sum_{l} P_{l}+I J K L \sum_{m} K_{m}+\right. \\
& +I J M \sum_{k l}(N P)_{k l}+I J L \sum_{k m}(N K)_{k m}+I J K \sum_{l m}(P K)_{l m}+J L M \sum_{i k}(E N)_{i k}+J K M \sum_{i l}(E P)_{i l}+ \\
& \left.+J K L \sum_{i m}(E K)_{i m}+J M \sum_{i k l}(E N P)_{i k l}+J L \sum_{i k m}(E N K)_{i k m}+J K \sum_{i l m}(E P K)_{i l m}+\sum_{i j k l m} e_{i j k l m}\right]^{2}
\end{aligned}
$$

assim, 


$$
\begin{aligned}
& \left(\sum_{k} y_{. . k_{i}}\right)^{2}=\left\{I^{2} J^{2} K^{2} L^{2} M^{2} \mu^{2}+J^{2} K^{2} L^{2} M^{2}\left(\sum_{i} E_{i}\right)^{2}+J^{2} L^{2} M^{2}\left[\sum_{i k}(E N)_{i k}\right]^{2}+\right. \\
& +J^{2} K^{2} M^{2}\left[\sum_{i l}(E P)_{i l}\right]^{2}+J^{2} K^{2} L^{2}\left[\sum_{i m}(E K)_{i m}\right]^{2}+J^{2} M^{2}\left[\sum_{i k l}(E N P)_{i k l}\right]^{2}+ \\
& \left.+J^{2} L^{2}\left[\sum_{i k m}(E N K)_{i k m}\right]^{2}+J^{2} K^{2}\left[\sum_{i l m}(E P K)_{i l m}\right]^{2}+\left[\sum_{i j k l m} e_{i j k l m}\right]^{2}+d p\right\}
\end{aligned}
$$

então,

$$
\begin{aligned}
& E\left(\sum_{k} y_{. . . . .}\right)^{2}=I^{2} J^{2} K^{2} L^{2} M^{2} \mu^{2}+J^{2} K^{2} L^{2} M^{2} I \sigma_{E}^{2}+J^{2} L^{2} M^{2} I K \sigma_{E N}^{2}+ \\
& +J^{2} K^{2} M^{2} I L \sigma_{E P}^{2}+J^{2} K^{2} L^{2} I M \sigma_{E K}^{2}+J^{2} M^{2} I K L \sigma_{E N P}^{2}+J^{2} L^{2} I K M \sigma_{E N K}^{2}+ \\
& +J^{2} K^{2} I L M \sigma_{E P K}^{2}+I J K I M \sigma^{2}
\end{aligned}
$$

logo,

$$
\begin{aligned}
& \frac{1}{I J K L M} E\left(\sum_{k} y_{. . . . .}\right)^{2}=I J K L M \mu^{2}+J K L M \sigma_{E}^{2}+J L M \sigma_{E N}^{e}+ \\
& +J K M \sigma_{E P}^{2}+J K L \sigma_{E K}^{2}+J M \sigma_{E N P}^{2}+J L \sigma_{E N K}^{2}+J K \sigma_{E P K}^{2}+\sigma^{2} .
\end{aligned}
$$

Subtraindo a expressão (2) da (3), chega-se que:

$$
\begin{aligned}
& \mathrm{E}(\mathrm{SQN})=I J L M \sum_{k} N_{k}^{2}+J L M(K-1) \sigma_{E N}^{2}+J M(K-1) \sigma_{E N P}^{2}+ \\
& +J L(K-1) \sigma_{E N K}^{2}+(K-1) \sigma^{2}
\end{aligned}
$$

portanto,

$$
E(Q M N)=\sigma^{2}+J L \sigma_{E N K}^{2}+J M \sigma_{E N P}^{2}+J L M \sigma_{E N}^{2}+I J L M \frac{\sum_{k} N_{k}^{2}}{K-1} .
$$

Analogamente, obtém-se as esperanças dos quadrados médios para os demais efeitos considerados no modelo, que estão resumidas na tabela 3. 
Tabela 3. Esperança dos quadrados médios para o modelo considerado na análise conjunta.

\begin{tabular}{l|l}
\hline Causas de Variação & \multicolumn{1}{c}{$\mathrm{E}(\mathrm{QM})$} \\
\hline $\mathrm{N}$ & $\sigma^{2}+J L \sigma_{E N K}^{2}+J M \sigma_{E N P}^{2}+J L M \sigma_{E N}^{2}+I J L M \phi_{N}$ \\
$\mathrm{P}$ & $\sigma^{2}+J M \sigma_{E N P}^{2}+J K \sigma_{E P K}^{2}+J K M \sigma_{E P}^{2}+I J K M \phi_{P}$ \\
$\mathrm{~K}$ & $\sigma^{2}+J L \sigma_{E N K}^{2}+J K \sigma_{E P K}^{2}+J K L \sigma_{E K}^{2}+I J K L \phi_{K}$ \\
$\mathrm{~N} \times \mathrm{P}$ & $\sigma^{2}+J M \sigma_{E N P}^{2}+I J M \phi_{N P}$ \\
$\mathrm{~N} \times \mathrm{K}$ & $\sigma^{2}+J L \sigma_{E N K}^{2}+I J L \phi_{N K}$ \\
$\mathrm{P} \times \mathrm{K}$ & $\sigma^{2}+J K \sigma_{E P K}^{2}+I J K \phi_{P K}$ \\
$\mathrm{E} \times \mathrm{N}$ & $\sigma^{2}+J L \sigma_{E N K}^{2}+J M \sigma_{E N P}^{2}+J L M \sigma_{E N}^{2}$ \\
$\mathrm{E} \times \mathrm{P}$ & $\sigma^{2}+J K \sigma_{E P K}^{2}+J M \sigma_{E N P}^{2}+J K M \sigma_{E P}^{2}$ \\
$\mathrm{E} \times \mathrm{K}$ & $\sigma^{2}+J K \sigma_{E P K}^{2}+J L \sigma_{E N K}^{2}+J K L \sigma_{E K}^{2}$ \\
$\mathrm{E} \times \mathrm{N} \times \mathrm{P}$ & $\sigma^{2}+J M \sigma_{E N P}^{2}$ \\
$\mathrm{E} \times \mathrm{N} \times \mathrm{K}$ & $\sigma^{2}+J L \sigma_{E N K}^{2}$ \\
$\mathrm{E} \times \mathrm{P} \times \mathrm{K}$ & $\sigma^{2}+J K \sigma_{E P K}^{2}$ \\
$\mathrm{Resíduo} \mathrm{Médio}$ & \\
\hline &
\end{tabular}

Onde:

$$
\begin{aligned}
& \phi_{N}=\frac{1}{K-1} \sum_{k} N_{k}^{2} ; \phi_{P}=\frac{1}{L-1} \sum_{l} P_{l}^{2} ; \phi_{K}=\frac{1}{M-1} \sum_{m} K_{m}^{2} ; \\
& \phi_{N P}=\frac{1}{(K-1)(L-1)} \sum_{k l}(N P)_{k l}^{2} ; \phi_{N K}=\frac{1}{(K-1)(M-1)} \sum_{k m}(N K)_{k m}^{2} \mathrm{e} \\
& \phi_{P K}=\frac{1}{(L-1)(M-1)} \sum_{l m}(P K)_{l m}^{2} .
\end{aligned}
$$




\subsubsection{Esperança dos quadrados médios para os componentes lineares e quadráticos de N, P e K}

No caso dos experimentos de adubação, existe interesse em determinar a curva de produção da cultura em estudo, em função das doses crescentes aplicadas de $\mathrm{N}$, $\mathrm{P}$ e K. Para isso, é usual decompor os efeitos de N, P e K em componentes lineares e quadráticos.

A técnica utilizada é a dos polinômios ortogonais, e designa-se como $c_{1 k}$ e $c_{2 k}$ os coeficientes dos polinômios linear e quadrático para o nitrogênio respectivamente. A notação utilizada para os níveis de $\mathrm{N}$ é $\mathrm{N}_{1}, \mathrm{~N}_{2}$, e $\mathrm{N}_{3}$, onde $\mathrm{N}_{1}$ corresponde ao nivel zero, $\mathrm{N}_{2}$ ao nivel um e $\mathrm{N}_{3}$ ao nível dois de $\mathrm{N}$, respectivamente. Analogamente para P e K.

A soma de quadrados para o efeito linear do nitrogênio é dada pela seguinte expressão :

$$
S Q\left(N^{\prime}\right)=\frac{1}{I J L M \sum c_{l k}^{2}}\left[\sum_{k} c_{1 k} y_{. . . . .}\right]^{2}, \quad \text { tal que } \sum_{k} c_{1 k}=0 .
$$

Assim,

$$
E\left[S Q\left(N^{\prime}\right)\right]=\frac{1}{I J L M \sum c_{1 k}^{2}} E\left[\sum_{k} c_{1 k} y_{. . . .}\right]^{2}
$$

onde,

$$
\begin{aligned}
& \sum_{k} c_{1 k} y_{. . k .}=\sum_{k} c_{1 k}\left(\sum_{i j l m} y_{i j k l m}\right)= \\
& =\sum_{k} c_{1 k}\left\{\sum_{i j l m}\left(\begin{array}{l}
\mu+E_{i}+b_{(i) j}+N_{k}+P_{l}+K_{m}+(N P)_{k l}+(N K)_{k m}+(P K)_{l m}+(E N)_{i k}+ \\
+(E P)_{i l}+(E K)_{i m}+(E N P)_{i k l}+(E N K)_{i k m}+(E P K)_{i l m}+e_{i j k l m}
\end{array}\right)\right\}=
\end{aligned}
$$




$$
\begin{aligned}
& =\sum_{k} c_{1 k}\left[I J M \mu+J L M \sum_{i} E_{i}+L M \sum_{i j} b_{(i) j}+I J L M N_{k}+I J M \sum_{i} P_{l}+I J L \sum_{m} K_{m}+\right. \\
& +I J M \sum_{l}(N P)_{k l}+I J L \sum_{m}(N K)_{k m}+I J \sum_{l m}(P K)_{l m}+J L M \sum_{i}(E N)_{i k}+J M \sum_{i l}(E P)_{i l}+ \\
& \left.+J L \sum_{i m}(E K)_{i m}+J M \sum_{i l}(E N P)_{i k l}+J L \sum_{i m}(E N K)_{i k m}+J \sum_{i l m}(E P K)_{i l m}+\sum_{i j l m} e_{i j k l m}\right]
\end{aligned}
$$

Impondo as restrições citadas anteriormente,

$$
\begin{aligned}
& \sum_{k} c_{1 k} y_{\ldots . . .}=I J L M \mu \sum_{k} c_{1 k}+J L M \sum_{i} E_{i} \sum_{k} c_{1 k}+I J L M \sum_{k} c_{1 k} N_{k}+ \\
& +J L M \sum_{k}\left[c_{1 k} \sum_{i}(E N)_{i k}\right]+J M \sum_{i l}(E P)_{i l} \sum_{k} c_{1 k}+J L \sum_{i m}(E K)_{i m} \sum_{k} c_{1 k}+ \\
& +J M \sum_{k}\left[c_{1 k} \sum_{i l}(E N P)_{i k l}\right]+J L \sum_{k}\left[c_{1 k} \sum_{i m}(E N K)_{i k m}\right]+ \\
& +J \sum_{i l m}(E P K)_{i l m} \sum_{k} c_{1 k}+\sum_{k}\left[c_{1 k} \sum_{i j l m} e_{i j k m}\right]
\end{aligned}
$$

e lembrando que $\sum_{k} c_{1 k}=0$, tem-se que,

$$
\begin{aligned}
& \sum_{k} c_{1 k} y_{\ldots . . .}=I J L M \sum_{k} c_{1 k} N_{k}+J L M \sum_{k}\left[c_{1 k} \sum_{i}(E N)_{i k}\right]+J M \sum_{k}\left[c_{1 k} \sum_{i l}(E N P)_{i k l}\right]+ \\
& +J L \sum_{k}\left[c_{1 k} \sum_{i m}(E N K)_{i k m}\right]+\sum_{k}\left[c_{1 k} \sum_{i j l m} e_{i j k l m}\right]
\end{aligned}
$$

portanto,

$$
\begin{aligned}
& {\left[\sum_{k} c_{1 k} y_{. . . . .}\right]^{2}=I^{2} J^{2} L^{2} M^{2}\left(\sum_{k} c_{1 k} N_{k}\right)^{2}+J^{2} L^{2} M^{2}\left[\sum_{k} c_{1 k}^{2} \sum_{i}(E N)_{i k}^{2}+d p\right]+} \\
& +J^{2} M^{2}\left[\sum_{k} c_{1 k}^{2} \sum_{l l}(E N P)_{i k l}^{2}+d p\right]+J^{2} L^{2}\left[\sum_{k} c_{1 k}^{2} \sum_{l m}(E N K)_{i k m}^{2}+d p\right]+ \\
& +\sum_{k} c_{1 k}^{2} \sum_{i l l m m} e_{i j k l m}^{2}+d p
\end{aligned}
$$


40

pois,

$$
\begin{aligned}
& \text { (i) }\left\{\sum_{k}\left[c_{1 k} \sum_{i}(E N)_{i k}\right]\right\}^{2}=\left\{\sum_{k}\left[c_{1 k}\left((E N)_{1 k}+(E N)_{2 k}+\cdots+(E N)_{I k}\right)\right]\right\}^{2}= \\
& =\left\{\sum_{k}\left[c_{1 k}(E N)_{1 k}+c_{1 k}(E N)_{2 k}+\cdots+c_{1 k}(E N)_{I k}\right]\right\}^{2}= \\
& =\left\{\sum_{k} c_{1 k}(E N)_{1 k}+\sum_{k} c_{1 k}(E N)_{2 k}+\cdots+\sum_{k} c_{1 k}(E N)_{I k}\right\}^{2}= \\
& =\left\{c_{11}(E N)_{11}+c_{12}(E N)_{12}+\cdots+c_{1 K}(E N)_{1 K}+\right. \\
& +c_{11}(E N)_{21}+c_{12}(E N)_{22}+\cdots+c_{1 K}(E N)_{2 K}+\cdots \\
& \left.\cdots+c_{11}(E N)_{I 1}+c_{12}(E N)_{I 2}+\cdots+c_{1 K}(E N)_{I K}\right\}^{2}= \\
& =c_{11}^{2}(E N)_{11}^{2}+c_{12}^{2}(E N)_{12}^{2}+\cdots+c_{1 K}^{2}(E N)_{1 K}^{2}+ \\
& +c_{11}^{2}(E N)_{21}^{2}+c_{12}^{2}(E N)_{22}^{2}+\cdots+c_{1 K}^{2}(E N)_{2 K}^{2}+\cdots \\
& \cdots+c_{11}^{2}(E N)_{I 1}^{2}+c_{12}^{2}(E N)_{I 2}^{2}+\cdots+c_{1 K}^{2}(E N)_{I K}^{2}+d p= \\
& =\sum_{k} c_{1 k}^{2} \sum_{i}(E N)_{i k}^{2}+d p .
\end{aligned}
$$

Analogamente,

(ii) $\left\{\sum_{k} c_{1 k}\left[\sum_{i l}(E N P)_{i k l}\right]\right\}^{2}=\sum_{k} c_{1 k}^{2} \sum_{i l}(E N P)_{i k l}^{2}+d p$;

(iii) $\left\{\sum_{k} c_{1 k}\left[\sum_{i m}(E N K)_{i k m}\right]\right\}^{2}=\sum_{k} c_{1 k}^{2} \sum_{i m}(E N K)_{i k m}^{2}+d p$;

(iv ) $\left[\sum_{k} c_{1 k}\left(\sum_{i j l m} e_{i j k l m}\right)\right]^{2}=\sum_{k} c_{1 k}^{2} \sum_{i j l m} e_{i j k l m}^{2}+d p$. 
Portanto,

$$
\begin{aligned}
& E\left[\sum_{k} c_{1 k} y_{. . k .}\right]^{2}=I^{2} J^{2} L^{2} M^{2}\left(\sum_{k} c_{1 k} N_{k}\right)^{2}+J^{2} L^{2} M^{2} \sum_{k} c_{1 k}^{2} I \sigma_{E N}^{2}+ \\
& +J^{2} M^{2} \sum_{k} c_{1 k}^{2} I L \sigma_{E N P}^{2}+J^{2} L^{2} \sum_{k} c_{1 k}^{2} I M \sigma_{E N K}^{2}+\sum_{k} c_{1 k}^{2} I J L M \sigma^{2}
\end{aligned}
$$

pois,

$$
E\left[\sum_{k} c_{1 k}^{2} \sum_{i}(E N)_{i k}^{2}+d p\right]=\sum_{k} c_{1 k}^{2} E\left[\sum_{i}(E N)_{i k}^{2}\right]=
$$$$
=\sum_{k} c_{1 k}^{2} E\left\{(E N)_{1 k}^{2}+(E N)_{2 k}^{2}+\cdots+(E N)_{1 k}^{2}\right\}=
$$$$
=\sum_{k} c_{1 k}^{2}(\underbrace{\sigma_{E N}^{2}+\sigma_{E N}^{2}+\cdots+\sigma_{E N}^{2}}_{I})=\sum_{k} c_{1 k}^{2} I \sigma_{E N}^{2} .
$$

Analogamente,

$$
E\left[\sum_{k} c_{1 k}^{2} \sum_{i l}(E N P)_{i k l}^{2}+d p\right]=\sum_{k} c_{1 k}^{2} E\left[\sum_{i l}(E N P)_{i k l}^{2}\right]=\sum_{k} c_{1 k}^{2} I L \sigma_{E N P}^{2}
$$

(iii ) $E\left[\sum_{k} c_{1 k}^{2} \sum_{i m}(E N K)_{i k m}^{2}+d p\right]=\sum_{k} c_{1 k}^{2} E\left[\sum_{i m}(E N K)_{i k m}^{2}\right]=\sum_{k} c_{1 k}^{2} I M \sigma_{E N K}^{2}$;

(iv) $E\left[\sum_{k} c_{1 k}^{2} \sum_{i j l m} e_{i j k l m}^{2}\right]=\sum_{k} c_{1 k}^{2} E\left[\sum_{i j l m} e_{i j k l m}^{2}\right]=\sum_{k} c_{1 k}^{2} I J L M \sigma^{2}$.

Portanto,

$$
E\left[S Q\left(N^{\prime}\right)\right]=\sigma^{2}+J L \sigma_{E N K}^{2}+J M \sigma_{E N P}^{2}+J L M \sigma_{E N}^{2}+\frac{I J L M}{\sum c_{1 k}^{2}}(\underbrace{\sum_{k} c_{1 k} N_{k}}_{Y\left(N^{\prime}\right)})^{2}=E\left[Q M\left(N^{\prime}\right)\right]
$$

pois, a $S Q\left(N^{\prime}\right)$ está associada a 1 grau de liberdade e $Y\left(N^{\prime}\right)$ é o valor teórico do contraste que corresponde ao efeito linear de $\mathrm{N}$. 
Para o conjunto de dados em questão, o fator $\mathrm{N}$ tem 3 niveis, os quais são quantitativos e os valores de $c_{l k}$ são referentes aos coeficientes do polinômio ortogonal de grau 1, isto é, $c_{11}=-1, c_{12}=0$ e $c_{13}=1$. Logo, chega-se que:

$$
\begin{aligned}
\left(\sum_{k} c_{1 k} N_{k}\right)^{2} & =\left(c_{11} N_{1}+c_{12} N_{2}+c_{13} N_{3}\right)^{2}= \\
& =\left[(-1) N_{1}+(0) N_{2}+(1) N_{3}\right]^{2}=\left(N_{3}-N_{1}\right)^{2}=\left[Y\left(N^{\prime}\right)\right]^{2} .
\end{aligned}
$$

Analogamente, utilizando $d_{1 l}$ como coeficientes do polinômio ortogonal para o efeito linear do fósforo e $e_{1 m}$ para o efeito linear do potássio, obtém-se:

$$
\begin{aligned}
& E\left[Q M\left(P^{\prime}\right)\right]=\sigma^{2}+J M \sigma_{E N P}^{2}+J K \sigma_{E P K}^{2}+J K M \sigma_{E P}^{2}+\frac{I J K M}{\sum d_{1 l}^{2}}(\underbrace{\sum_{i} d_{1 l} P_{l}}_{Y\left(P^{\prime}\right)})^{2} ; \\
& E\left[Q M\left(K^{\prime}\right)\right]=\sigma^{2}+J L \sigma_{E N K}^{2}+J K \sigma_{E P K}^{2}+J K L \sigma_{E K}^{2}+\frac{I J K L}{\sum e_{1 m}^{2}}(\underbrace{\sum_{m} e_{1 m} K_{m}}_{Y\left(K^{\prime}\right)})^{2} .
\end{aligned}
$$

$Y\left(P^{\prime}\right)$ e $Y\left(K^{\prime}\right)$ são os valores teóricos dos contrastes que correspondem aos efeitos lineares de $\mathrm{P}$ e $\mathrm{K}$, respectivamente.

Para obter as esperanças dos quadrados médios para os efeitos quadráticos de N, P e K, basta substituir os coeficientes lineares $c_{l k}, d_{l l}$ e $e_{l m}$ pelos coeficientes quadráticos $c_{2 k}, d_{2 l}$ e $e_{2 m}$, respectivamente. 


\subsubsection{Esperança dos quadrados médios para $N^{\prime} \times P^{\prime}, N^{\prime} \times K^{\prime}$ e $P^{\prime} \times K^{\prime}$.}

Nos experimentos de adubação, com a finalidade de verificar se o efeito de um nutriente tem influência sobre o efeito do outro, existe interesse em estudar as interações de primeira ordem entre os nutrientes utilizados. As interações de interesse prático neste caso, são somente aquelas entre os efeitos lineares, isto é, $N^{\prime} \times P^{\prime}, N^{\prime} \times K^{\prime}$ e $\mathrm{P}^{\prime} \times \mathrm{K}^{\prime}$. As interações do tipo $\mathrm{N}^{\prime} \times \mathrm{P}^{\prime \prime}, \mathrm{N}^{\prime} \times \mathrm{P}^{\prime}, \ldots, \mathrm{P}^{\prime \prime} \times \mathrm{K}^{\prime \prime}$, não são de interesse prático neste tipo de estudo.

\subsubsection{Obtenção da $\mathrm{E}\left[Q \mathrm{Q}\left(\mathrm{N}^{\prime} \times \mathrm{P}^{\prime}\right)\right]$}

Para a interação $N^{\prime} \times \mathrm{P}^{\prime}$ a $E\left[Q M\left(N^{\prime} \times P^{\prime}\right)\right]=E\left[S Q\left(N^{\prime} \times P^{\prime}\right)\right]$, pois a $S Q\left(N^{\prime} \times P^{\prime}\right)$ está associada a apenas 1 grau de liberdade e é obtida pela seguinte expressão:

$$
S Q\left(N^{\prime} \times P^{\prime}\right)=\frac{1}{I J M \sum_{k l}\left(c_{1 k} d_{1 l}\right)^{2}}\left[\sum_{k l} c_{1 k} d_{1 l} y_{. . k l .}\right]^{2}
$$

tal que $\sum_{k, l} c_{1 k} d_{1 l}=0$ e $\sum_{k} c_{1 k}=\sum_{l} d_{1 l}=0$

logo,

$$
E\left[S Q\left(N^{\prime} \times P^{\prime}\right)\right]=\frac{1}{I J M \sum_{k l}\left(c_{1 k} d_{1 l}\right)^{2}} E\left[\sum_{k l} c_{1 k} d_{1 l} y_{. k l .}\right]^{2}
$$


Considerando $\mathrm{N}$ e $\mathrm{P}$ com 3 níveis cada um, têm-se que os coeficientes do polinômio ortogonal de grau 1 , são: $c_{l k}(k=1,2,3) \Rightarrow-1,0$ e 1 e $d_{l l}(l=1,2,3) \Rightarrow-1,0$ e 1 . Assim, os valores referentes ao produto de $c_{1 k} d_{1 l}$, são obtidos da seguinte maneira:

\begin{tabular}{ccc|ccc} 
& & & & & \\
& & & $\mathrm{d}_{11}$ & $\mathrm{~d}_{12}$ & $\mathrm{~d}_{13}$ \\
& & & -1 & 0 & 1 \\
\hline \multirow{3}{*}{$\mathrm{N} \quad \mathrm{c}_{11}$} & -1 & 1 & 0 & -1 \\
& $c_{12}$ & 0 & 0 & 0 & 0 \\
& $c_{13}$ & 1 & -1 & 0 & 1
\end{tabular}

Da expressão (4), obtém-se que:

$$
\begin{aligned}
& \sum_{k l} c_{1 k} d_{1 l} y_{. . k l .}=\sum_{k l} c_{1 k} d_{1 l}\left(\sum_{i j m} y_{i j k l m}\right)= \\
& =\sum_{k l} c_{1 k} d_{1 l}\left\{\sum_{i j m}\left(\begin{array}{l}
\mu+E_{i}+b_{(i) j}+N_{k}+P_{l}+K_{m}+(N P)_{k l}+(N K)_{k m}+(P K)_{l m}+(E N)_{i k}+ \\
+(E P)_{i l}+(E K)_{i m}+(E N P)_{i k l}+(E N K)_{i k m}+(E P K)_{i l m}+e_{i j k l m}
\end{array}\right)\right\}= \\
& =\sum_{k l} c_{1 k} d_{1 l}\left[I J M \mu+J M \sum_{i} E_{i}+M \sum_{i j} b_{(i) j}+I J M N_{k}+I J M P_{l}+I J \sum_{m} K_{m}+\right. \\
& +I J M(N P)_{k l}+I J \sum_{m}(N K)_{k m}+I J \sum_{m}(P K)_{l m}+J M \sum_{i}(E N)_{i k}+J M \sum_{i}(E P)_{i l}+ \\
& \left.+J \sum_{i m}(E K)_{i m}+J M \sum_{i}(E N P)_{i k l}+J \sum_{i m}(E N K)_{i k m}+J \sum_{i m}(E P K)_{l l m}+\sum_{i j m} e_{i j k l m}\right] .
\end{aligned}
$$

Impondo as restrições citadas anteriormente, 


$$
\begin{aligned}
& \sum_{k l} c_{1 k} d_{1 l} y_{. k l .}=I J M \mu \sum_{k l} c_{1 k} d_{1 l}+J M \sum_{i} E_{i} \sum_{k l} c_{1 k} d_{1 l}+I J M \sum_{k l} c_{1 k} d_{1 l} N_{k}+ \\
& +I J M \sum_{k l} c_{1 k} d_{1 l} P_{l}+I J M \sum_{k l} c_{1 k} d_{1 l}(N P)_{k l}+J M \sum_{k l}\left[c_{1 k} d_{1 l} \sum_{i}(E N)_{i k}\right]+ \\
& +J M \sum_{k l}\left[c_{1 k} d_{1 l} \sum_{i}(E P)_{i l}\right]+J \sum_{i m}(E K)_{i m} \sum_{k l} c_{1 k} d_{1 l}+J M \sum_{k l}\left[c_{1 k} d_{1 l} \sum_{i}(E N P)_{i k l}\right]+ \\
& +J \sum_{k l}\left[c_{1 k} d_{1 l} \sum_{i m}(E N K)_{i k m}\right]+J \sum_{k l}\left[c_{1 k} d_{1 l} \sum_{i m}(E P K)_{i l m}\right]+\sum_{k l}\left[c_{1 k} d_{1 l} \sum_{i j m} e_{i j k l m}\right]= \\
& =I J M \mu \sum_{k l} c_{1 k} d_{1 l}+J M \sum_{i} E_{i} \sum_{k l} c_{1 k} d_{1 l}+I J M\left(\sum_{k} c_{1 k} N_{k}\right) \sum_{l} d_{1 l}+I J M\left(\sum_{l} d_{1 l} P_{l}\right) \sum_{k} c_{1 k}+ \\
& +I J M\left(\sum_{k l} c_{1 k} d_{1 l}(N P)_{k l}\right)+J M\left(\sum_{k} c_{1 k} \sum_{i}(E N)_{i k}\right) \sum_{l} d_{1 l}+J M\left(\sum_{l} d_{1 l} \sum_{i}(E P)_{i l}\right) \sum_{k} c_{1 k}+ \\
& +J\left(\sum_{i m}(E K)_{i m}\right) \sum_{k l} c_{1 k} d_{1 l}+J M\left(\sum_{k l} c_{1 k} d_{1 l} \sum_{i}(E N P)_{i k l}\right)+J\left(\sum_{k} c_{1 k} \sum_{i m}(E N K)_{i k m}\right) \sum_{l} d_{1 l}+ \\
& +J\left(\sum_{l} d_{1 l} \sum_{i m}(E P K)_{i l m}\right) \sum_{k} c_{1 k}+\sum_{k l} c_{1 k} d_{1 l} \sum_{i j m} e_{i j k l m},
\end{aligned}
$$

e lembrando que $\sum_{k, l} c_{1 k} d_{1 l}=0$ e $\sum_{k} c_{1 k}=\sum_{l} d_{1 l}=0$, tem-se que,

$$
\sum_{k l} c_{1 k} d_{1 l} y_{. . k l .}=I J M\left(\sum_{k l} c_{1 k} d_{1 l}(N P)_{k l}\right)+J M\left(\sum_{k l} c_{1 k} d_{1 l} \sum_{i}(E N P)_{i k l}\right)+\sum_{k l} c_{1 k} d_{1 l} \sum_{i j m} e_{i j k l m}
$$

logo,

$$
\begin{aligned}
& {\left[\sum_{k l} c_{1 k} d_{1 l} y_{. . k l .}\right]^{2}=I^{2} J^{2} M^{2}\left[\sum_{k l} c_{1 k} d_{1 l}(N P)_{k l}\right]^{2}+J^{2} M^{2} \sum_{k l}\left(c_{1 k} d_{1 l}\right)^{2} \sum_{i}(E N P)_{i k l}^{2}+} \\
& +\sum_{k l}\left(c_{1 k} d_{1 l}\right)^{2} \sum_{i j m} e_{i j k l m}^{2}+d p
\end{aligned}
$$

e 


$$
\begin{aligned}
& E\left[\sum_{k l} c_{1 k} d_{1 l} y_{. k l .}\right]^{2}=I^{2} J^{2} M^{2} E\left[\sum_{k l} c_{1 k} d_{1 l}(N P)_{k l}\right]^{2}+J^{2} M^{2} \sum_{k l}\left(c_{1 k} d_{1 l}\right)^{2} E\left[\sum_{i}(E N P)_{i k l}^{2}\right]+ \\
& +\sum_{k l}\left(c_{1 k} d_{1 l}\right)^{2} E\left[\sum_{i j m} e_{i j k l m}^{2}\right]= \\
& =I^{2} J^{2} M^{2}\left[\sum_{k l} c_{1 k} d_{1 l}(N P)_{k l}\right]^{2}+J^{2} M^{2} \sum_{k l}\left(c_{1 k} d_{1 l}\right)^{2} I \sigma_{E N P}^{2}+\sum_{k l}\left(c_{1 k} d_{1 l}\right)^{2} I J M \sigma^{2} .
\end{aligned}
$$

Como,

$$
E\left[S Q\left(N^{\prime} \times P^{\prime}\right)\right]=\frac{1}{I J M \sum_{k l}\left(c_{1 k} d_{1 l}\right)^{2}} E\left[\sum_{k l} c_{1 k} d_{1 l} y_{. k l .}\right]^{2}
$$

portanto,

$$
E\left[S Q\left(N^{\prime} \times P^{\prime}\right)\right]=\sigma^{2}+J M \sigma_{E N P}^{2}+\frac{I J M}{\sum_{k l}\left(c_{1 k} d_{1 l}\right)^{2}}[\underbrace{\sum_{k l} c_{1 k} d_{1 l}(N P)_{k l}}_{Y\left(N^{\prime} \times P^{\prime}\right)}]^{2}=E\left[Q M\left(N^{\prime} \times P^{\prime}\right)\right]
$$

Analogamente, para N' $\times K^{\prime}$ e $P^{\prime} \times K^{\prime}$, obtêm-se:

$$
\begin{aligned}
& E\left[Q M\left(N^{\prime} \times K^{\prime}\right)\right]=\sigma^{2}+J L \sigma_{E N K}^{2}+\frac{I J L}{\sum_{k m}\left(c_{1 k} e_{1 m}\right)^{2}}[\underbrace{\sum_{k m} c_{1 k} e_{1 m}(N K)_{k m}}_{Y\left(N^{\prime} \times K^{\prime}\right)}]^{2} ; \\
& E\left[Q M\left(P^{\prime} \times K^{\prime}\right)\right]=\sigma^{2}+J K \sigma_{E P K}^{2}+\frac{I J K}{\sum_{l m}\left(d_{1 l} e_{1 m}\right)^{2}}[\underbrace{\sum_{l m} d_{1 l} e_{1 m}(P K)_{l m}}_{Y\left(P^{\prime} \times K^{\prime}\right)}]^{2} .
\end{aligned}
$$

$$
Y\left(N^{\prime} \times P^{\prime}\right), Y\left(N^{\prime} \times K^{\prime}\right) \text { e } Y\left(P^{\prime} \times K^{\prime}\right) \text { são os valores teóricos dos contrastes }
$$

que correspondem aos efeitos de $N^{\prime} \times P^{\prime}, N^{\prime} \times K^{\prime}$ e $P^{\prime} \times K^{\prime}$, respectivamente. 


\subsubsection{Esperança dos quadrados médios para as interações $E \times N^{\prime}, E \times P^{\prime} e E \times K^{\prime}$}

Demonstra-se que o $\mathrm{QM}\left(\mathrm{E} \times \mathrm{N}^{\prime}\right)$ é o resíduo específico para testar o efeito linear do nitrogênio ( $\left.N^{\prime}\right)$. A soma de quadrados para $E \times N^{\prime}$ é dada pela seguinte expressão:

$$
S Q\left(E \times N^{\prime}\right)=\frac{1}{J L M \sum_{k} c_{1 k}^{2}} \sum_{i}\left[\sum_{k} c_{1 k} y_{i . k . .}\right]^{2}-S Q\left(N^{\prime}\right), \text { tal que } \sum_{k} c_{1 k}=0
$$

Assim,

$$
E\left[S Q\left(E \times N^{\prime}\right)\right]=\frac{1}{J L M \sum_{k} c_{1 k}^{2}} E\left[\sum_{i}\left(\sum_{k} c_{1 k} y_{i . k . .}\right)^{2}\right]-E\left[S Q\left(N^{\prime}\right)\right]
$$

onde,

$$
\begin{aligned}
& \sum_{k} c_{1 k} y_{i, k . .}=\sum_{k} c_{1 k}\left(\sum_{j l m} y_{i j k l m}\right)= \\
& =\sum_{k} c_{1 k}\left\{\sum_{j l m}\left(\begin{array}{l}
\mu+E_{i}+b_{(i) \jmath}+N_{k}+P_{l}+K_{m}+(N P)_{k l}+(N K)_{k m}+(P K)_{l m}+(E N)_{i k}+ \\
+(E P)_{i l}+(E K)_{i m}+(E N P)_{i k l}+(E N K)_{i k m}+(E P K)_{i l m}+e_{i j k l m}
\end{array}\right)=\right. \\
& =\sum_{k} c_{1 k}\left[J L M \mu+J L M E_{i}+L M \sum_{j} b_{(i) j}+J L M N_{k}+J M \sum_{l} P_{l}+J L \sum_{m} K_{m}+\right. \\
& +J M \sum_{l}(N P)_{k l}+J L \sum_{m}(N K)_{k m}+J \sum_{l m}(P K)_{l m}+J L M(E N)_{i k}+J M \sum_{l}(E P)_{i l}+ \\
& \left.+J L \sum_{m}(E K)_{i m}+J M \sum_{l}(E N P)_{i k l}+J L \sum_{m}(E N K)_{i k m}+J \sum_{l m}(E P K)_{i l m}+\sum_{j l m} e_{i j k l m}\right]
\end{aligned}
$$


Impondo as restrições citadas anteriormente,

$$
\begin{aligned}
& \sum_{k} c_{1 k} y_{i . k . .}=J L M \mu \sum_{k} c_{1 k}+J L M E_{i} \sum_{k} c_{1 k}+J L M \sum_{k} c_{1 k} N_{k}+J L M \sum_{k}\left[c_{1 k}(E N)_{i k}\right]+ \\
& +J M \sum_{l}(E P)_{i l} \sum_{k} c_{1 k}+J L \sum_{m}(E K)_{i m} \sum_{k} c_{1 k}+J M \sum_{k}\left[c_{1 k} \sum_{l}(E N P)_{i k l}\right]+ \\
& +J L \sum_{k}\left[c_{1 k} \sum_{m}(E N K)_{i k m}\right]+J \sum_{l m}(E P K)_{i l m} \sum_{k} c_{1 k}+\sum_{k}\left[c_{1 k} \sum_{j l m} e_{i j k l m}\right],
\end{aligned}
$$

e lembrando que $\sum_{k} c_{1 k}=0$, tem-se que,

$$
\begin{aligned}
& \sum_{k} c_{1 k} y_{i . k . .}=J L M \sum_{k} c_{1 k} N_{k}+J L M \sum_{k}\left[c_{1 k}(E N)_{i k}\right]+J M \sum_{k}\left[c_{1 k} \sum_{l}(E N P)_{i k l}\right]+ \\
& +J L \sum_{k}\left[c_{1 k} \sum_{m}(E N K)_{i k m}\right]+\sum_{k}\left[c_{1 k} \sum_{j l m} e_{i j k l m}\right]
\end{aligned}
$$

logo,

$$
\begin{aligned}
& {\left[\sum_{k} c_{1 k} y_{i . k . .}\right]^{2}=J^{2} L^{2} M^{2}\left(\sum_{k} c_{1 k} N_{k}\right)^{2}+J^{2} L^{2} M^{2}\left[\sum_{k} c_{1 k}^{2}(E N)_{i k}^{2}+d p\right]+} \\
& +J^{2} M^{2}\left[\sum_{k} c_{1 k}^{2} \sum_{l}(E N P)_{i k l}^{2}+d p\right]+J^{2} L^{2}\left[\sum_{k} c_{1 k}^{2} \sum_{m}(E N K)_{i k m}^{2}+d p\right]+ \\
& +\sum_{k} c_{1 k}^{2} \sum_{j l m} e_{i j k l m}^{2}+d p
\end{aligned}
$$

$\circ$

$$
\begin{aligned}
& \sum_{i}\left(\sum_{k} c_{1 k} y_{i \cdot k \cdot .}\right)^{2}=I J^{2} L^{2} M^{2}\left(\sum_{k} c_{1 k} N_{k}\right)^{2}+J^{2} L^{2} M^{2}\left[\sum_{k} c_{1 k}^{2} \sum_{i}(E N)_{i k}^{2}+d p\right]+ \\
& +J^{2} M^{2}\left[\sum_{k} c_{1 k}^{2} \sum_{l l}(E N P)_{i k l}^{2}+d p\right]+J^{2} L^{2}\left[\sum_{k} c_{1 k}^{2} \sum_{i m}(E N K)_{i k m}^{2}+d p\right]+ \\
& +\sum_{k} c_{1 k}^{2} \sum_{i j l m} e_{i j k l m}^{2}+d p .
\end{aligned}
$$


Chega-se então, que

$$
\begin{aligned}
& E\left[\sum_{i}\left(\sum_{k} c_{1 k} y_{i, k . .}\right)^{2}\right]=I^{2} L^{2} M^{2}\left(\sum_{k} c_{1 k} N_{k}\right)^{2}+J^{2} L^{2} M^{2} \sum_{k} c_{1 k}^{2} I \sigma_{E N}^{2}+ \\
& +J^{2} M^{2} \sum_{k} c_{1 k}^{2} I L \sigma_{E N P}^{2}+J^{2} L^{2} \sum_{k} c_{1 k}^{2} I M \sigma_{E N K}^{2}+\sum_{k} c_{1 k}^{2} I J L M \sigma^{2}
\end{aligned}
$$

pois,

$$
\begin{aligned}
& \text { (i) } E\left[\sum_{k} c_{1 k}^{2} \sum_{i}(E N)_{i k}^{2}+d p\right]=\sum_{k} c_{1 k}^{2} E\left[\sum_{i}(E N)_{i k}^{2}\right]= \\
& =\sum_{k} c_{1 k}^{2} E\left\{(E N)_{1 k}^{2}+(E N)_{2 k}^{2}+\cdots+(E N)_{l k}^{2}\right\}= \\
& =\sum_{k} c_{1 k}^{2}\left(\sigma_{E N}^{2}+\sigma_{E N}^{2}+\cdots+\sigma_{E N}^{2}\right)=\sum_{k} c_{1 k}^{2} I \sigma_{E N}^{2}
\end{aligned}
$$

Analogamente,

(ii) $E\left[\sum_{k} c_{1 k}^{2} \sum_{i l}(E N P)_{i k l}^{2}+d p\right]=\sum_{k} c_{1 k}^{2} E\left[\sum_{i l}(E N P)_{i k l}^{2}\right]=\sum_{k} c_{1 k}^{2} I L \sigma_{E N P}^{2}$;

(iii ) $E\left[\sum_{k} c_{1 k}^{2} \sum_{i m}(E N X)_{i k m}^{2}+d p\right]=\sum_{k} c_{1 k}^{2} E\left[\sum_{i m}(E N K)_{i k m}^{2}\right]=\sum_{k} c_{1 k}^{2} I M \sigma_{E N K}^{2}$;

(iv) $E\left[\sum_{k} c_{1 k}^{2} \sum_{i j l m} e_{i j k l m}^{2}\right]=\sum_{k} c_{1 k}^{2} E\left[\sum_{i j l m} e_{i j k l m}^{2}\right]=\sum_{k} c_{1 k}^{2} I J L M \sigma^{2}$.

Consequentemente,

$$
\begin{aligned}
& \frac{1}{J L M \sum_{k} c_{1 k}^{2}} E\left[\sum_{i}\left(\sum_{k} c_{i k} y_{i . k . .}\right)^{2}\right]=I \sigma^{2}+I J L \sigma_{E N K}^{2}+I J M \sigma_{E N P}^{2}+ \\
& +I J L M \sigma_{E N}^{2}+\frac{I J L M}{\sum_{k} c_{1 k}^{2}}\left(\sum_{k} c_{1 k} N_{k}\right)^{2} .
\end{aligned}
$$

Como,

$$
E\left[S Q\left(E \times N^{\prime}\right)\right]=\frac{1}{J L M \sum_{k} c_{1 k}^{2}} E\left[\sum_{i}\left(\sum_{k} c_{1 k} y_{i . k . .}\right)^{2}\right]-E\left[S Q\left(N^{\prime}\right)\right]=(5)-E\left[S Q\left(N^{\prime}\right)\right]
$$


onde,

$E\left[S Q\left(N^{\prime}\right)\right]=\sigma^{2}+J L \sigma_{E N K}^{2}+J M \sigma_{E N P}^{2}+J L M \sigma_{E N}^{2}+\frac{I J L M}{\sum c_{1 k}^{2}}\left(\sum_{k} c_{1 k} N_{k}\right)^{2}$

logo,

$E\left[S Q\left(E \times N^{\prime}\right)\right]=(I-1) \sigma^{2}+(I-1) J L \sigma_{E N K}^{2}+(I-1) J M \sigma_{E N P}^{2}+(I-1) J L M \sigma_{E N}^{2}$.

Sabendo-se que,

$E\left[Q M\left(E \times N^{\prime}\right)\right]=E\left[\frac{S Q\left(E \times N^{\prime}\right)}{I-1}\right]=\frac{1}{I-1} E\left[S Q\left(E \times N^{\prime}\right)\right]$

portanto,

$E\left[Q M\left(E \times N^{\prime}\right)\right]=\sigma^{2}+J L \sigma_{E N K}^{2}+J M \sigma_{E N P}^{2}+J L M \sigma_{E N}^{2}$.

Analogamente, para o fósforo tem-se que,

$E\left[Q M\left(E \times P^{\prime}\right)\right]=\sigma^{2}+J M \sigma_{E N P}^{2}+J K \sigma_{E P K}^{2}+J K M \sigma_{E P}^{2}$

e para o potássio,

$E\left[Q M\left(E \times K^{\prime}\right)\right]=\sigma^{2}+J L \sigma_{E N K}^{2}+J K \sigma_{E P S}^{2}+J K L \sigma_{E K}^{2}$.

4.1.4 Esperança dos quadrados médios para $E \times N^{\prime} \times P^{\prime}, E \times N^{\prime} \times K^{\prime}$ e $E \times P^{\prime} \times K^{\prime}$

Demonstra-se que o $\mathrm{QM}\left(\mathrm{E} \times \mathrm{N}^{\prime} \times \mathrm{P}^{\prime}\right)$ é o resíduo específico para testar a interação $N^{\prime} \times P^{\prime}$. A soma de quadrados para $E \times N^{\prime} \times P^{\prime}$ é dada pela seguinte expressão:

$$
S Q\left(E \times N^{\prime} \times P^{\prime}\right)=\frac{1}{J M \sum_{k l}\left(c_{1 k} d_{1 l}\right)^{2}} \sum_{i}\left(\sum_{k l} c_{1 k} d_{1 l} y_{i . k l .}\right)^{2}-S Q\left(N^{\prime} \times P^{\prime}\right)
$$

tal que, $\sum_{k, l} c_{1 k} d_{1 l}=0$ e $\sum_{k} c_{1 k}=\sum_{l} d_{1 l}=0$ 
Assim,

$$
E\left[S Q\left(E \times N^{\prime} \times P^{\prime}\right)\right]=\frac{1}{J M \sum_{k l}\left(c_{1 k} d_{1 l}\right)^{2}} E\left[\sum_{i}\left(\sum_{k l} c_{1 k} d_{1 l} y_{i, k l .}\right)^{2}\right]-E\left[S Q\left(N^{\prime} \times P^{\prime}\right)\right]
$$

onde,

$$
\begin{aligned}
& \sum_{k l} c_{1 k} d_{1 l} y_{i, k l .}=\sum_{k l} c_{1 k} d_{1 l}\left(\sum_{j m} y_{i j k l m}\right)= \\
& =\sum_{k l} c_{1 k} d_{1 l}\left\{\sum_{j m}\left(\begin{array}{l}
\mu+E_{i}+b_{(i) j}+N_{k}+P_{l}+K_{m}+(N P)_{k l}+(N K)_{k m}+(P K)_{l m}+(E N)_{i k}+ \\
+(E P)_{i l}+(E K)_{i m}+(E N P)_{i k l}+(E N K)_{i k m}+(E P K)_{i l m}+e_{i j k l m}
\end{array}\right)\right\}= \\
& =\sum_{k l} c_{1 k} d_{1 l}\left[J M \mu+J M E_{i}+M \sum_{j} b_{(i) j}+J M N_{k}+J M P_{l}+J \sum_{m} K_{m}+\right. \\
& +J M(N P)_{k l}+J \sum_{m}(N K)_{k m}+J \sum_{m}(P K)_{l m}+J M(E N)_{i k}+J M(E P)_{i l}+ \\
& \left.+J \sum_{m}(E K)_{i m}+J M(E N P)_{i k l}+J \sum_{m}(E N K)_{i k m}+J \sum_{m}(E P K)_{i l m}+\sum_{j m} e_{i j k l m}\right]
\end{aligned}
$$

Impondo as restrições citadas anteriormente,

$$
\begin{aligned}
& \sum_{k l} c_{1 k} d_{1 l} y_{i . k l .}=J M \mu \sum_{k l} c_{1 k} d_{1 l}+J M E_{i} \sum_{k l} c_{1 k} d_{1 l}+J M\left(\sum_{k} c_{1 k} N_{k}\right) \sum_{l} d_{1 l}+ \\
& +J M\left(\sum_{l} d_{1 l} P_{l}\right) \sum_{k} c_{1 k}+J M\left(\sum_{k l} c_{1 k} d_{1 l}(N P)_{k l}\right)+J M\left(\sum_{k} c_{1 k}(E N)_{i k}\right) \sum_{l} d_{1 l}+ \\
& +J M\left(\sum_{l} d_{1 l}(E P)_{i l}\right) \sum_{k} c_{1 k}+J\left(\sum_{m}(E K)_{i m}\right) \sum_{k l} c_{1 k} d_{1 l}+J M\left(\sum_{k l} c_{1 k} d_{1 l}(E N P)_{i k l}\right)+ \\
& +J\left(\sum_{k} c_{1 k} \sum_{m}(E N K)_{i k m}\right) \sum_{l} d_{1 l}+J\left(\sum_{l} d_{1 l} \sum_{m}(E P K)_{i l m}\right) \sum_{k} c_{1 k}+\sum_{k l} c_{1 k} d_{1 l} \sum_{j m} e_{i j k l m}
\end{aligned}
$$

e lembrando que, $\sum_{k, l} c_{1 k} d_{1 l}=0$ e $\sum_{k} c_{1 k}=\sum_{l} d_{1 l}=0$, tem-se que, 


$$
\sum_{k l} c_{1 k} d_{1 l} y_{i . k l .}=J M\left(\sum_{k l} c_{1 k} d_{1 l}(N P)_{k l}\right)+J\left(\sum_{k l} c_{1 k} d_{1 l}(E N P)_{i k l}\right)+\sum_{k l} c_{1 k} d_{1 l} \sum_{j m} e_{i j k l m}
$$

logo,

$$
\begin{aligned}
& \left(\sum_{k l} c_{1 k} d_{1 l} y_{i, k l .}\right)^{2}=J^{2} M^{2}\left[\sum_{k l} c_{1 k} d_{1 l}(N P)_{k l}\right]^{2}+J^{2} M^{2} \sum_{k l}\left(c_{1 k} d_{1 l}\right)^{2}(E N P)_{i k l}^{2}+ \\
& +\sum_{k l}\left(c_{1 k} d_{1 l}\right)^{2} \sum_{j m} e_{i j k l m}^{2}+d p \\
& \sum_{i}\left(\sum_{k l} c_{1 k} d_{1 l} y_{i . k l .}\right)^{2}=I J^{2} M^{2}\left[\sum_{k l} c_{1 k} d_{1 l}(N P)_{k l}\right]^{2}+J^{2} M^{2} \sum_{k l}\left(c_{1 k} d_{1 l}\right)^{2} \sum_{i}(E N P)_{i k l}^{2}+ \\
& +\sum_{k l}\left(c_{1 k} d_{1 l}\right)^{2} \sum_{i j m} e_{i j k l m}^{2}+d p
\end{aligned}
$$

e

$$
\begin{aligned}
& E\left[\sum_{i}\left(\sum_{k l} c_{1 k} d_{1 l} y_{i \cdot k l .}\right)^{2}\right]=I J^{2} M^{2} E\left[\sum_{k l} c_{1 k} d_{1 l}(N P)_{k l}\right]^{2}+ \\
& +J^{2} M^{2} \sum_{k l}\left(c_{1 k} d_{1 l}\right)^{2} E\left[\sum_{l}(E N P)_{i k l}^{2}\right]+\sum_{k l}\left(c_{1 k} d_{1 l}\right)^{2} E\left[\sum_{i j m} e_{i j k l m}^{2}\right]= \\
& =I J^{2} M^{2}\left[\sum_{k l} c_{1 k} d_{1 l}(N P)_{k l}\right]^{2}+J^{2} M^{2} \sum_{k l}\left(c_{1 k} d_{1 l}\right)^{2} I \sigma_{E N P}^{2}+\sum_{k l}\left(c_{1 k} d_{1 l}\right)^{2} I J M \sigma^{2} .
\end{aligned}
$$

Consequentemente,

$$
\begin{aligned}
& \frac{1}{J M \sum_{k l}\left(c_{1 k} d_{1 l}\right)^{2}} E\left[\sum_{i}\left(\sum_{k l} c_{1 k} d_{l l} y_{i, k l}\right)^{2}\right]= \\
& =I \sigma^{2}+I M \sigma_{E N P}^{2}+\frac{I J M}{\sum_{k l}\left(c_{1 k} d_{1 l}\right)^{2}}\left[\sum_{k l} c_{1 k} d_{1 l}(N P)_{k l}\right]^{2} .
\end{aligned}
$$


Da expressão (6), tem-se que,

$$
E\left[S Q\left(E \times N^{\prime} \times P^{\prime}\right)\right]=(7)-E\left[S Q\left(N^{\prime} \times P^{\prime}\right)\right]
$$

onde,

$$
E\left[S Q\left(N^{\prime} \times P^{\prime}\right)\right]=\sigma^{2}+J M \sigma_{E N P}^{2}+\frac{I J M}{\sum_{k l}\left(c_{1 k} d_{1 l}\right)^{2}}\left[\sum_{k l} c_{1 k} d_{1 l}(N P)_{k l}\right]^{2}
$$

logo,

$E\left[S Q\left(E \times N^{\prime} \times P^{\prime}\right)\right]=(I-1) \sigma^{2}+(I-1) J M \sigma_{E N P}^{2}$.

Sabendo-se que,

$E\left[Q M\left(E \times N^{\prime} \times P^{\prime}\right)\right]=\frac{1}{I-1} E\left[S Q\left(E \times N^{\prime} \times P^{\prime}\right)\right]$

portanto,

$E\left[Q M\left(E \times N^{\prime} \times P^{\prime}\right)\right]=\sigma^{2}+J M \sigma_{E N P}^{2}$.

Analogamente, para $E \times N^{\prime} \times K^{\prime}$ tem-se que,

$E\left[Q M\left(E \times N^{\prime} \times K^{\prime}\right)\right]=\sigma^{2}+J L \sigma_{E N K}^{2}$

e para $E \times \mathrm{P}^{\prime} \times \mathrm{K}^{\prime}$,

$E\left[Q M\left(E \times P^{\prime} \times K^{\prime}\right)\right]=\sigma^{2}+J K \sigma_{E P K}^{2}$. 


\subsection{Testes para homogeneidade de variâncias}

As análises individuais foram realizadas com a finalidade de obter os quadrados médios dos resíduos, os quais estão apresentados na tabela 4 .

Tabela 4. Quadrados Médios dos Resíduos para as análises individuais

\begin{tabular}{c|c|c}
\hline Experimentos & Q.M. & G.L. \\
\hline 1 & 118,41 & 15 \\
3 & 61,95 & 15 \\
4 & 104,58 & 15 \\
5 & 117,82 & 15 \\
6 & 280,02 & 15 \\
7 & 470,91 & 15 \\
8 & 231,96 & 15 \\
9 & 302,49 & 15 \\
10 & 608,83 & 15 \\
11 & 250,25 & 15 \\
\hline
\end{tabular}

Com esses valores, verificou-se a hipótese de homogeneidade de variâncias entre experimentos, através dos seguintes testes:

\subsubsection{Teste de Hartley}

O teste de Hartley, de acordo com Milliken \& Johnson (1992), é construido da seguinte maneira:

$$
F_{\max }=\frac{s_{\max }^{2}}{s_{\min }^{2}}=\frac{608,83}{61,95}=9,83 ;
$$




$$
F_{\alpha, v, k}=F_{0,05,15,11}=5,77 .
$$

Portanto, rejeita-se a hipótese de homogeneidade de variâncias, pois,

$$
F_{\max }=9,83>F_{\alpha, v, k}=5,77 \text {. }
$$

\subsubsection{Teste de Bartlett}

O teste de Bartlett de acordo com Snedecor \& Cochran (1967), é obtido através de:

$$
\begin{aligned}
M & =2,3026(n)\left(k \log \bar{s}^{2}-\sum_{i} \log s_{i}^{2}\right)= \\
& =2,3026(15)[11(2,4426)-25,8045]
\end{aligned}
$$

onde,

$$
\bar{s}^{2}=\frac{\sum s_{i}^{2}}{k}=\frac{3047,58}{11}=277,05
$$

então,

$$
\begin{aligned}
& M=2,3026(15)(1,0641)=36,7529 ; \\
& C=1+\frac{k+1}{3 k n}=1+\frac{11+1}{3(11)(15)}=1,0242 ;
\end{aligned}
$$

logo,

$$
\chi_{c}^{2}=\frac{M}{C}=\frac{36,7529}{1,0242}=35,88 .
$$

O valor tabelado de chi-quadrado é:

$$
\chi_{(k-1)}^{2}=18,31 \text {. }
$$

Portanto, rejeita-se a hipótese de homogeneidade de variâncias ao nivel de $5 \%$ de probabilidade, pois, $\chi_{c}^{2}=35,88>\chi_{(k-1)}^{2}=18,31$. 


\subsection{Método de Cochran para ajuste dos graus de liberdade}

Devido a existência de heterocedasticidade entre os experimentos, como foi verificado em 4.2, aplicou-se o método de Cochran para ajuste dos graus de liberdade na análise de variância.

Para o resíduo médio, o número de graus de liberdade é dado por:

$$
n_{r}=\frac{\left(\sum_{i} s_{i}^{2}\right)^{2}}{\sum_{i}\left(s_{i}^{2}\right)^{2}} \text {, cujo valor obtido è } n_{r}=118,89 \cong 119
$$

onde, $s_{i}^{2}$ é o quadrado médio do resíduo e $n_{i}$ é o número de graus de liberdade para o experimento $i$, respectivamente.

Para a interação experimento com efeitos de tratamentos, os números de graus de liberdade foram obtidos através da seguinte forma geral, adaptado por Pimentel Gomes (1987).

$$
n_{E \times T}=\frac{(T-1)(I-1)^{2} V_{1}^{2}}{(I-2) V_{2}+V_{1}^{2}}
$$

onde,

$(T-1)$ é o número de graus de liberdade para o efeito considerado;

$I$ é o número de experimentos.

Assim, para o conjunto de dados que está sendo analisado, o número de graus de liberdade para a interação $\mathrm{E} \times \mathrm{N}$ é dado por:

$$
n_{E \times N}=\frac{(3-1)(11-1)^{2}(277,05)^{2}}{(11-2) \cdot 106529,04+(277,05)^{2}}=14,82 \cong 15
$$

onde,

$$
V_{1}=\frac{\sum s_{i}^{2}}{I}=\frac{3047,59}{11}=277,05 \mathrm{e}
$$




$$
V_{2}=\frac{\sum\left(s_{i}^{2}\right)^{2}}{I}=\frac{1171819,45}{11}=106529,04
$$

Analogamente para $n_{E \times P}$ e $n_{E \times K}$, obtêm-se que:

$$
n_{E \times P}=n_{E \times K}=14,82 \cong 15 \text {. }
$$

\subsection{Análise de variância}

O esquema da análise de variância para o modelo proposto é dado pela tabela 5 .

\begin{tabular}{|c|c|c|}
\hline Causas de Variação & G.L. & Q.M. \\
\hline Experimentos (E) & $(\mathrm{I}-1)=\mathrm{n}_{1}$ & $\mathrm{~V}_{1}$ \\
\hline Blocos d. E & $\mathrm{I}(\mathrm{J}-1)=\mathrm{n}_{2}$ & $\mathrm{~V}_{2}$ \\
\hline $\mathrm{N}$ & $(K-1)=n_{3}$ & $\mathrm{~V}_{3}$ \\
\hline $\mathbf{P}$ & $(L-1)=n_{4}$ & $\mathrm{~V}_{4}$ \\
\hline $\mathrm{K}$ & $(M-1)=n_{5}$ & $\mathrm{~V}_{5}$ \\
\hline$N^{\prime} \times P^{\prime}$ & $1=n_{6}$ & $\mathrm{~V}_{6}$ \\
\hline$N^{\prime} \times K^{\prime}$ & $1=n_{7}$ & $\mathrm{~V}_{7}$ \\
\hline$P^{\prime} \times K^{\prime}$ & $1=n_{8}$ & $\mathrm{~V}_{8}$ \\
\hline $\mathrm{E} \times \mathrm{N}$ & $(I-1)(K-1)=n_{9}$ & $\mathrm{~V}_{9}$ \\
\hline$E \times P$ & $(I-1)(L-1)=n_{10}$ & $\mathrm{~V}_{10}$ \\
\hline $\mathrm{E} \times \mathrm{K}$ & $(I-1)(M-1)=n_{11}$ & $\mathrm{~V}_{11}$ \\
\hline$E \times N^{\prime} \times P^{\prime}$ & $(I-1) .1=n_{12}$ & $\mathrm{~V}_{12}$ \\
\hline$E \times N^{\prime} \times K^{\prime}$ & $(I-1) \cdot 1=n_{13}$ & $\mathrm{~V}_{13}$ \\
\hline $\mathrm{E} \times \mathrm{P}^{\prime} \times \mathrm{K}^{\prime}$ & $(I-1) .1=n_{14}$ & $\mathrm{~V}_{14}$ \\
\hline Resíduo Médio & I. $15=\mathrm{n}_{15}$ & $\mathrm{~V}_{15}$ \\
\hline
\end{tabular}

Tabela 5. Esquema da análise de variância. 
$\mathrm{O}$ teste $\mathrm{F}$, aplicado a análise de variância, tem os valores obtidos de acordo com os resultados das respectivas esperanças dos quadrados médios, apresentados em 4.1, cujos resultados são mostrados na tabela 6 .

Tabela 6. Análise de variância conjunta.

\begin{tabular}{|c|c|c|c|c|c|}
\hline Causas de Variação & G.L. & S.Q. & Q.M. & $F$ & $\operatorname{Pr}>F$ \\
\hline Experimentos (E) & 10 & 1185325,87 & 118532,59 & & \\
\hline Blocos d. E & 22 & 10989,36 & 499,52 & & \\
\hline $\mathrm{N}$ & 2 & 3931,39 & 1965,70 & 5,02 & 0,0214 \\
\hline$P$ & 2 & 643,38 & 321,69 & 1,84 & 0,1929 \\
\hline $\mathrm{K}$ & 2 & 12741,20 & 6370,60 & 12,13 & 0,0007 \\
\hline$N^{\prime} \times P^{\prime}$ & 1 & 39,82 & 39,82 & 0,17 & 0,6888 \\
\hline$N^{\prime} \times K^{\prime}$ & 1 & 29,69 & 29,69 & 0,09 & 0,7703 \\
\hline $\mathrm{P}^{\prime} \times \mathrm{K}^{\prime}$ & 1 & 30,07 & 30,07 & 0,12 & 0,7362 \\
\hline $\mathrm{E} \times \mathrm{N}$ & $20(15)$ & 7824,07 & 391,20 & 1,30 & 0,4137 \\
\hline $\mathrm{E} \times \mathrm{P}$ & $20(15)$ & 3495,72 & 174,79 & 0,85 & 0,6482 \\
\hline $\mathrm{E} \times \mathrm{K}$ & $20(15)$ & 10505,16 & 525,26 & 1,73 & 0,2837 \\
\hline $\mathrm{E} \times \mathrm{N}^{\prime} \times \mathrm{P}^{\prime}$ & 10 & 2390,66 & 239,07 & 0,86 & 0,5725 \\
\hline $\mathrm{E} \times \mathrm{N}^{\prime} \times \mathrm{K}^{\prime}$ & 10 & 3380,58 & 338,06 & 1,22 & 0,2852 \\
\hline $\mathrm{E} \times \mathrm{P}^{\prime} \times \mathrm{K}^{\prime}$ & 10 & 2424,80 & 242,48 & 0,88 & 0,5539 \\
\hline Resíduo Médio & $165(119)$ & 45713,82 & 277,05 & & \\
\hline
\end{tabular}

Os números entre parênteses são referentes ao número de graus de liberdade ajustados pelo método de Cochran e considerados no cálculo da $\operatorname{Pr}>\mathrm{F}$.

Os valores de F, na tabela 6 , foram obtidos seguindo a designação para os quadrados médios apresentada na tabela 5 . Os critérios utilizados foram os seguintes: 
1. Teste F para Nitrogênio

$$
F=\frac{V_{3}}{V_{9}}=\frac{1965,70}{391,20}=5,02 \quad \text { com } n_{3}=2 \text { e } n_{9}=15 \text { graus de liberdade; }
$$

2. Teste F para Fósforo

$$
F=\frac{V_{4}}{V_{10}}=\frac{321,69}{174,79}=1,84 \text { com } n_{4}=2 \text { e } n_{10}=15 \text { graus de liberdade; }
$$

3. Teste F para Potássio

$$
F=\frac{V_{s}}{V_{11}}=\frac{6370,60}{525,26}=12,13 \quad \text { com } n_{5}=2 \text { e } n_{11}=15 \text { graus de liberdade; }
$$

4. Teste $F$ para a Interação $N^{\prime} \times P^{\prime}$

$$
F=\frac{V_{6}}{V_{12}}=\frac{39,82}{239,07}=0,17 \text { com } n_{6}=1 \text { e } n_{12}=10 \text { graus de liberdade; }
$$

5. Teste $F$ para a Interação $N^{\prime} \times K^{\prime}$

$$
F=\frac{V_{7}}{V_{13}}=\frac{29,69}{338,06}=0,09 \text { com } n_{7}=1 \text { e } n_{13}=10 \text { graus de liberdade }
$$

6. Teste $F$ para a Interação $P^{\prime} \times K^{\prime}$

$$
F=\frac{V_{8}}{V_{14}}=\frac{30,07}{242,48}=0,12 \text { com } n_{8}=1 \text { e } n_{14}=10 \text { graus de liberdade; }
$$


7. Teste $F$ para a Interação $\mathrm{E} \times \mathrm{N}$

$$
F=\frac{V_{9}}{V_{12}+V_{13}-V_{15}}=\frac{391,20}{300,08}=1,30 \quad \operatorname{com} v_{1} \text { e } v_{2} \text { graus de liberdade; }
$$

onde, $v_{1}=n_{9}=15$ e $v_{2}$ é obtido por Satterthwaite (1946), da seguinte forma:

$$
v_{2}=\frac{\left(V_{12}+V_{13}-V_{15}\right)}{\frac{\left(V_{12}\right)^{2}}{n_{12}}+\frac{\left(V_{13}\right)^{2}}{n_{13}}+\frac{\left(V_{15}\right)^{2}}{n_{15}}}=\frac{(300,08)^{2}}{17788,92}=5,06 ;
$$

8. Teste $\mathrm{F}$ para a Interação $\mathrm{E} \times \mathbf{P}$

$$
F=\frac{V_{10}}{V_{12}+V_{14}-V_{15}}=\frac{174,79}{204,05}=0,85 \quad \operatorname{com} v_{3} \text { e } v_{4} \text { graus de liberdade; }
$$

onde, $v_{3}=n_{10}=15$ e $v_{4}$ é obtido por Satterthwaite (1946), da seguinte forma:

$$
v_{4}=\frac{\left(V_{12}+V_{14}-V_{15}\right)^{2}}{\frac{\left(V_{12}\right)^{2}}{n_{12}}+\frac{\left(V_{14}\right)^{2}}{n_{14}}+\frac{\left(V_{15}\right)^{2}}{n_{15}}}=\frac{(204,5)^{2}}{12240,12}=3,42 ;
$$

9. Teste $\mathrm{F}$ para a Interação $\mathrm{E} \times \mathrm{K}$

$$
F=\frac{V_{11}}{V_{13}+V_{14}-V_{15}}=\frac{525,26}{303,49}=1,73 \quad \text { com } v_{5} \text { e } v_{6} \text { graus de liberdade }
$$

onde, $v_{5}=n_{11}=15$ e $v_{6}$ é obtido por Satterthwaite (1946), da seguinte forma: 


$$
v_{6}=\frac{\left(V_{13}+V_{14}-V_{15}\right)^{2}}{\frac{\left(V_{13}\right)^{2}}{n_{13}}+\frac{\left(V_{14}\right)^{2}}{n_{14}}+\frac{\left(V_{15}\right)^{2}}{n_{15}}}=\frac{(303,49)^{2}}{17953,13}=5,13 ;
$$

10. Teste $F$ para a Interação $E \times N^{\prime} \times P^{\prime}$

$$
F=\frac{V_{12}}{V_{15}}=\frac{239,07}{277,05}=0,86 \text { com } n_{12}=10 \text { e } n_{15}=119 \text { graus de liberdade; }
$$

11. Teste $F$ para a Interação $E \times N^{\prime} \times K^{\prime}$

$$
F=\frac{V_{13}}{V_{15}}=\frac{338,06}{277,05}=1,22 \quad \text { com } n_{13}=10 \text { e } n_{15}=119 \text { graus de liberdade; }
$$

12. Teste $F$ para a Interação $E \times P^{\prime} \times K^{\prime}$

$$
F=\frac{V_{14}}{V_{15}}=\frac{242,48}{277,05}=0,88 \quad \text { com } n_{14}=10 \text { e } n_{15}=119 \text { graus de liberdade }
$$

Pela tabela 6, verifica-se que todas as interações envolvendo experimentos não foram significativas, indicando que pode-se obter uma conclusão geral para a região considerada.

As interações de primeira ordem $N^{\prime} \times \mathrm{P}^{\prime}, \mathrm{N}^{\prime} \times \mathrm{K}^{\prime}$ e $\mathrm{P}^{\prime} \times \mathrm{K}^{\prime}$, também não foram significativas, indicando que o efeito de um nutriente independe do efeito do outro.

Os efeitos para $\mathrm{N}$ e $\mathrm{K}$ foram significativos. Decorrente disso, houve interesse na decomposição dos dois graus de liberdade associados a soma de quadrados de cada um deles, pois, em cada caso, um grau de liberdade pode ser tomado como referente ao efeito linear e o outro, ao efeito quadrático do fertilizante. As somas de quadrados das interações $\mathrm{E} \times \mathrm{N}$ e $\mathrm{E} \times \mathrm{K}$ também foram decompostas para a obtenção de 
um resíduo específico, com a finalidade de testar cada um dos componentes com o componente obtido das interações $\mathrm{E} \times \mathrm{N}$ e $\mathrm{E} \times \mathrm{K}$.

As análises foram realizadas com o uso do pacote SAS. Porém, o SAS não oferece a técnica do resíduo específico como opção. Então, foi feito um programa (apêndice 2) com o objetivo de gerar as somas de quadrados correspondentes aos efeitos $N^{\prime}(E), N^{\prime}(E)$ e para os demais efeitos considerados. Fazendo uso da propriedade dos espaços ortogonais, $S Q N^{\prime}+S Q\left(E \times N^{\prime}\right)=S Q N^{\prime}(E)$, obteve-se por diferença a $\mathrm{SQ}\left(\mathrm{E} \times \mathrm{N}^{\prime}\right)$. Estes resultados são apresentados na tabela 7.

Tabela 7. Análise de variância utilizando o resíduo específico.

\begin{tabular}{|c|c|c|c|c|c|}
\hline Causas de Variação & G.L. & S.Q. & Q.M. & $F$ & $\operatorname{Pr}>F$ \\
\hline $\mathrm{N}^{\prime}$ & 1 & 3703,2813 & 3703,2813 & 14,97 & 0,0031 \\
\hline$N^{\prime}$ & 1 & 228,1105 & 228,1105 & 0,43 & 0,5269 \\
\hline $\mathrm{P}^{\prime}$ & 1 & 624,7116 & 624,7116 & 3,72 & 0,0826 \\
\hline$P^{\prime \prime}$ & 1 & 18,6668 & 18,6668 & 0,10 & 0,7583 \\
\hline $\mathrm{K}^{\prime}$ & 1 & 12126,0114 & 12126,0114 & 13,85 & 0,0040 \\
\hline$K^{\prime \prime}$ & 1 & 615,1856 & 615,1856 & 3,51 & 0,0905 \\
\hline $\mathrm{E} \times \mathrm{N}^{\prime}$ & 10 & 2473,5104 & 247,3510 & & \\
\hline$E \times N^{\prime}$ & 10 & 5350,5601 & 535,0560 & & \\
\hline$E \times P^{\prime}$ & 10 & 1678,7390 & 167,8739 & & \\
\hline$E \times P^{\prime \prime}$ & 10 & 1816,9804 & 181,6980 & & \\
\hline$E \times K^{\prime}$ & 10 & 8754,2925 & 875,4293 & & \\
\hline $\mathrm{E} \times \mathrm{K}^{\prime \prime}$ & 10 & 1750,8705 & 175,0871 & & \\
\hline
\end{tabular}

Os testes para N', N', $\mathrm{P}^{\prime}, \mathrm{P}^{\prime}, \mathrm{K}^{\prime}$ e $\mathrm{K}$ " foram realizados com os respectivos resíduos específicos, pois, observa-se na tabela 7 , uma diferença relativamente grande nas variâncias das interações $E \times N\left(E \times N ', E \times N^{\prime}\right.$ ') e $E \times K$ $\left(E \times K^{\prime}, E \times K^{\prime \prime}\right)$. O efeito de $P$ não foi significativo, mas aparece na tabela 7 a título de ilustração. 
Nota-se diferenças significativas quando se realiza o teste $\mathrm{F}$ considerando os resíduos específicos. $\mathrm{O}$ efeito $\mathrm{N}^{\prime}$ quando testado utilizando o $\mathrm{QM}(\mathrm{E} \times \mathrm{N})$ no denominador, produziu $F=9,47$ e quando testado utilizando o $\mathrm{QM}\left(\mathrm{E} \times \mathrm{N}^{\prime}\right)$ produziu $\mathrm{F}=14,97$. Para o efeito $\mathrm{K}^{\prime}$, utilizando $\mathrm{QM}(\mathrm{E} \times \mathrm{K})$ no denominador, produziu $\mathrm{F}=23,09$ e com $\mathrm{QM}\left(\mathrm{E} \times \mathrm{K}^{\prime}\right)$ produziu $\mathrm{F}=13,85$, como pode ser verificado nas tabelas 7 e 8 .

Tabela 8. Análise de variância não utilizando o resíduo específico.

\begin{tabular}{lcrrrr}
\hline Causas de Variação & G.L. & \multicolumn{1}{c}{ S.Q. } & \multicolumn{1}{c}{ Q.M. } & \multicolumn{1}{c}{ F } & Pr > F \\
\hline N' & 1 & 3703,2813 & 3703,2813 & 9,47 & 0,0077 \\
N' & 1 & 228,1105 & 228,1105 & 0,58 & 0,4581 \\
P' & 1 & 624,7116 & 624,7116 & 3,57 & 0,0783 \\
P’ & 1 & 18,6668 & 18,6668 & 0,11 & 0,7447 \\
K' & 1 & 12126,0114 & 12126,0114 & 23,09 & 0,0002 \\
K' & 1 & 615,1856 & 615,1856 & 1,17 & 0,2965 \\
\hline E $\times$ N & $20(15)$ & 7824,0705 & 391,2035 & & \\
E $\times$ P & $20(15)$ & 3495,7194 & 174,7860 & & \\
E $\times$ K & $20(15)$ & 10505,1630 & 525,2582 & & \\
\hline
\end{tabular}

Observa-se, nas tabelas 7 e 8 , que na composição do teste $F$, por exemplo para N'e N" com o $\mathrm{QM}(\mathrm{E} \times \mathrm{N})$ no denominador, considera-se o valor médio entre $\mathrm{QM}\left(\mathrm{E} \times \mathrm{N}^{\prime}\right)$ e $\mathrm{QM}\left(\mathrm{E} \times \mathrm{N}^{\prime \prime}\right)$. Se estes valores são relativamente diferentes, o denominador com o $\mathrm{QM}(\mathrm{E} \times \mathrm{N})$ não é o mais adequado.

$O$ conjunto de dados utilizado ilustra bem o fato de que, quando não existe grandes diferenças nas variâncias da interação, não há necessidade de fazer a decomposição, como se observa para $\mathrm{E} \times \mathrm{P}^{\prime} \mathrm{e} \mathrm{E} \times \mathrm{P}$ ', na tabela 7 . E quando existe uma relativa diferença nas variâncias da interação, verifica-se que o teste mais adequado é o composto com os resíduos especificos correspondentes, como se observa para $E \times N^{\prime}$, $E \times N^{\prime \prime}$ e $E \times K^{\prime}, E \times K^{\prime \prime}$, na tabela 7 . 


\subsection{Teste de combinação de probabilidades}

Nos experimentos agronômicos, espera-se que os efeitos de adubação com nitrogênio, fósforo e potássio sejam positivos. O teste de combinação de probabilidades, quando aplicado, é unilateral à direita. Então, para os contrastes de interesse referentes aos efeitos lineares e quadráticos de $\mathrm{N}, \mathrm{P}$ e $\mathrm{K}$ espera-se que:

$$
\begin{aligned}
& \hat{Y}\left(N^{\prime}\right)=N_{3}-N_{1}>0 ; \\
& \hat{Y}\left(P^{\prime}\right)=P_{3}-P_{1}>0 \\
& \hat{Y}\left(K^{\prime}\right)=K_{3}-K_{1}>0 ; \\
& \hat{Y}\left(N^{\prime \prime}\right)=2 N_{2}-N_{1}-N_{3}>0 \Rightarrow N_{2}>\frac{N_{1}+N_{3}}{2}, \text { ou seja, dose média }
\end{aligned}
$$

de $N_{2}$ maior do que $\frac{N_{1}+N_{3}}{2}$;

$$
\begin{aligned}
& \hat{Y}\left(P^{\prime \prime}\right)=2 P_{2}-P_{1}-P_{3}>0 ; \\
& \hat{Y}\left(K^{\prime \prime}\right)=2 K_{2}-K_{1}-K_{3}>0 .
\end{aligned}
$$

No teste de combinação de probabilidades, se $\chi_{2 n}^{2}$ for significativo, é uma indicação de que na grande maioria dos locais o contraste de interesse teve sinais de significância, e no conjunto, o contraste é significativo.

Os cálculos para os testes envolvendo os contrates lineares são apresentados na tabela 9 . 


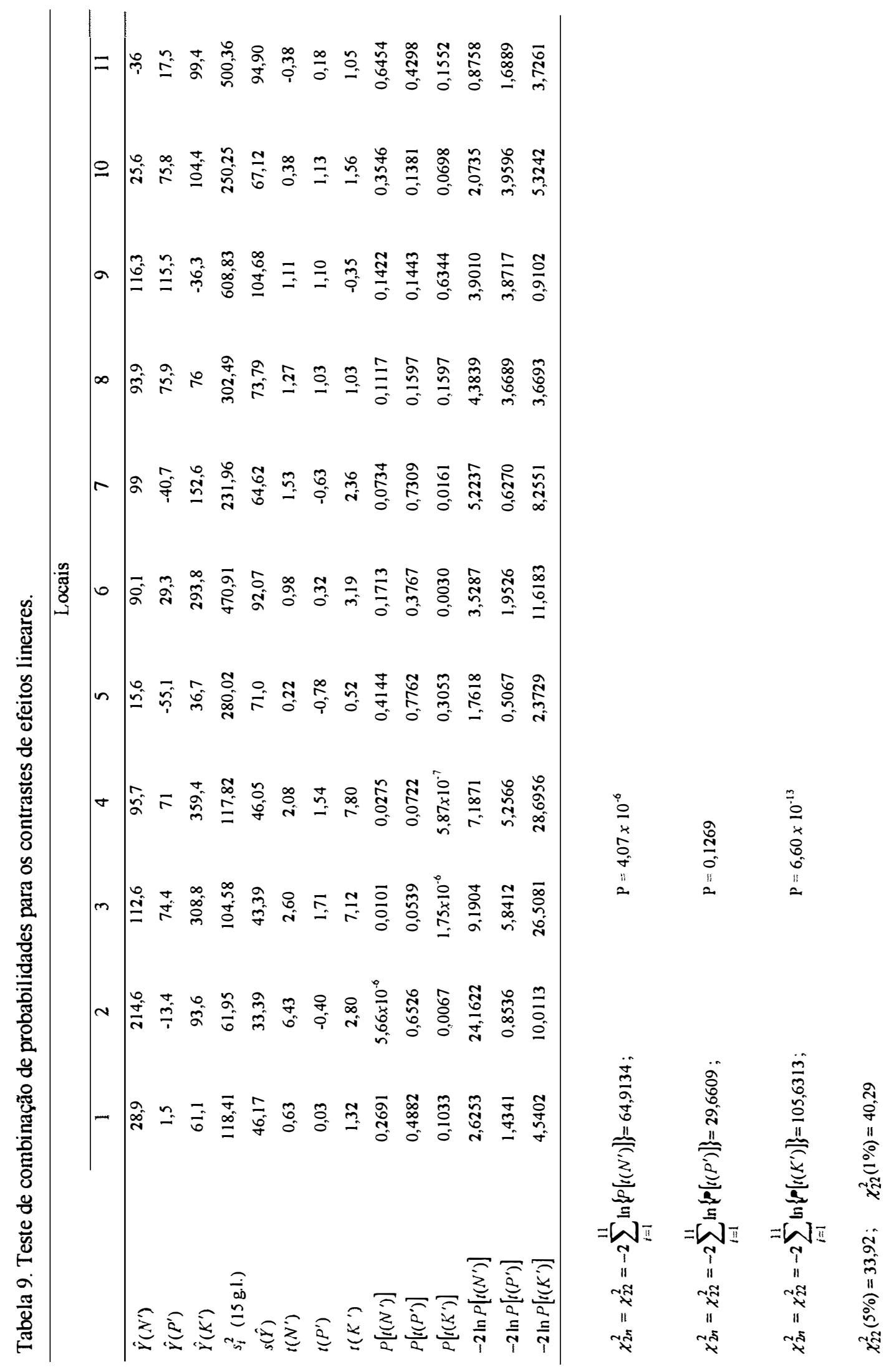


Os valores de $s(\hat{Y})$, para os contrastes dos efeitos lineares, foram obtidos da seguinte forma:

$$
s(\hat{Y})=\sqrt{\left\{(1)^{2} 9+(-1)^{2} 9\right\} s^{2}}=\sqrt{(9+9) s^{2}}=\sqrt{18 s^{2}}
$$

onde, o 9 é o número de parcelas para obter cada total $\left(\mathrm{N}_{1}, \mathrm{~N}_{2}, \ldots, \mathrm{K}_{3}\right)$.

Com os resultados obtidos da tabela 9 , verifica-se que os contrastes $\hat{Y}\left(N^{\prime}\right)$ e $\hat{Y}\left(K^{\prime}\right)$ foram significativos, indicando que o efeito médio geral para $\mathrm{N}^{\prime}$ e $\mathrm{K}$ ' foi significativo. Estes resultados levam a conclusões coincidentes com os obtidos com $o$ teste $F$ da tabela 7 , que foram obtidos, nos testes dos contrastes, com os seus erros específicos.

Os cálculos para os testes envolvendo os contrates quadráticos são apresentados na tabela 10. 


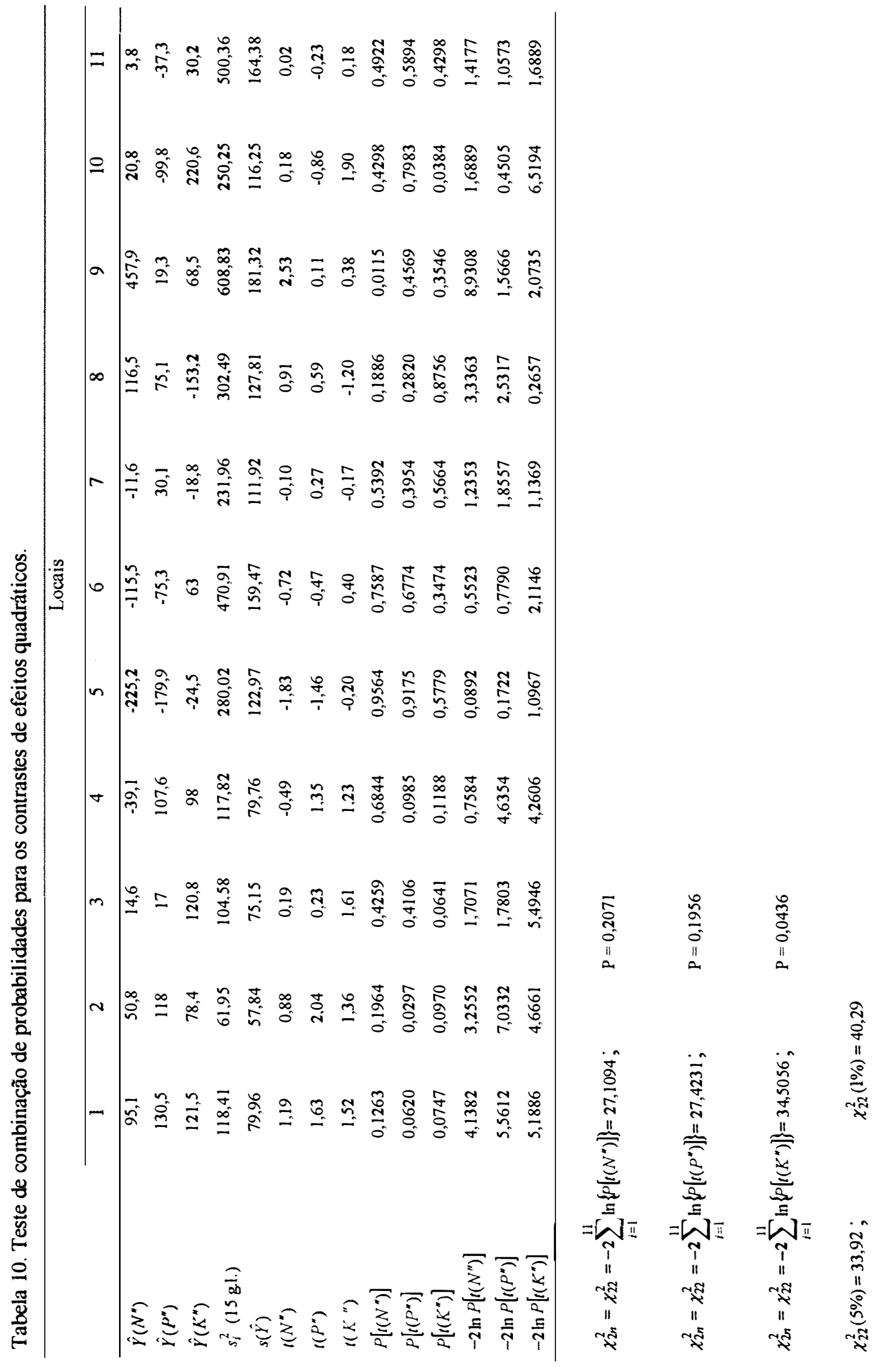


Os valores de $s(\hat{Y})$, para os contrastes dos efeitos quadráticos, foram obtidos da seguinte forma:

$$
s(\hat{Y})=\sqrt{\left\{(2)^{2} 9+(-1)^{2} 9+(-1)^{2} 9\right\} s^{2}}=\sqrt{(36+9+9) s^{2}}=\sqrt{54 s^{2}}
$$

Os resultados obtidos da tabela 10 , são praticamente os mesmos que os obtidos na análise de variância com o teste F (tabela 7). Uma pequena diferença ocorreu para o efeito do $K^{\prime}$, onde pelo teste $\mathrm{F}$, na análise de variância, obteve-se $\mathrm{P}=0,0905 \mathrm{e}$ pelo teste de combinação de probabilidades, obteve-se $P=0,0436$. Esta diferença pode ser pelo fato do teste de combinação de probabilidades ser baseado no erro experimental único, enquanto que o teste $\mathrm{F}$ foi baseado no erro especifico de cada contraste.

\subsection{Análise de variância ponderada}

As tabelas 6, 7 e 8 foram construidas com os resultados das análises não ponderadas, isto é, os pesos atribuídos a cada valor observado são iguais para todos os experimentos e além disso, foram feitos ajustes nos números de graus de liberdade, utilizando o método de Cochran (1954), para contornar o problema da heterogeneidade de variâncias entre os experimentos.

Um outro método proposto, para este problema, é a análise de variância ponderada, em que as ponderações são inversamente proporcionais às estimativas das variâncias dos experimentos individuais.

$\mathrm{O}$ teste $\mathrm{F}$, para a análise de variância ponderada, foi realizado de acordo com as esperanças dos quadrados médios, apresentadas em 4.1. Os resultados são apresentados na tabela 11 . 
Tabela 11. Análise de variância ponderada.

\begin{tabular}{lccccc}
\hline Causas de Variação & G.L. & S.Q. & Q.M. & F & $\operatorname{Pr}>$ F \\
\hline Experimentos (E) & 10 & 78,7081 & 7,8708 & & \\
Blocos d. E & 22 & 0,2980 & 0,0135 & & \\
$\mathrm{~N}$ & 2 & 0,2363 & 0,1182 & 3,91 & 0,0369 \\
$\mathrm{P}$ & 2 & 0,0297 & 0,0149 & 1,94 & 0,1698 \\
$\mathrm{~K}$ & 2 & 0,5690 & 0,2845 & 7,63 & 0,0034 \\
$\mathrm{~N}^{\prime} \times \mathrm{P}^{\prime}$ & 1 & 0,0002 & 0,0002 & 0,03 & 0,8659 \\
$\mathrm{~N}^{\prime} \times \mathrm{K}^{\prime}$ & 1 & 0,0016 & 0,0016 & 0,31 & 0,5899 \\
$\mathrm{P}^{\prime} \times \mathrm{K}^{\prime}$ & 1 & 0,0005 & 0,0005 & 0,13 & 0,7259 \\
$\mathrm{E} \times \mathrm{N}^{\prime}$ & 20 & 0,6046 & 0,0302 & 5,12 & 0,0393 \\
$\mathrm{E} \times \mathrm{P}$ & 20 & 0,1538 & 0,0077 & 1,64 & 0,3397 \\
$\mathrm{E} \times \mathrm{K}^{\prime}$ & 20 & 0,7451 & 0,0373 & 10,97 & 0,0359 \\
$\mathrm{E} \times \mathrm{N}^{\prime} \times \mathrm{P}^{\prime}$ & 10 & 0,0653 & 0,0065 & 1,12 & 0,3501 \\
$\mathrm{E} \times \mathrm{N}^{\prime} \times \mathrm{K}^{\prime}$ & 10 & 0,0520 & 0,0052 & 0,90 & 0,5346 \\
$\mathrm{E} \times \mathrm{P}^{\prime} \times \mathrm{K}^{\prime}$ & 10 & 0,0396 & 0,0040 & 0,69 & 0,7328 \\
Residuo Médio & 165 & 0,9506 & 0,0058 & & \\
\hline Total & 296 & 82,4544 & & &
\end{tabular}

Pela tabela 11 verifica-se que os efeitos de $E \times N$ e $E \times K$ foram significativos ao nivel de $5 \%$ de probabilidade, indicando que os efeitos de $\mathrm{N}$ e $\mathrm{K}$ têm um comportamento diferenciado dentro de cada experimento. Deve-se, então, estudar estes efeitos isoladamente dentro de cada local. O programa SAS, elaborado para a análise de variância ponderada, encontra-se no apêndice 3 .

Verificou-se, na análise não ponderada, que o efeito médio geral de N'e $\mathrm{K}$ ' foi significativo, enquanto que na análise ponderada, os efeitos de $\mathrm{N}$ e $\mathrm{K}$ têm um comportamento diferenciado de local para local. Estas diferenças ocorreram devido ao fato de que a análise ponderada é mais sensivel, pois, é realizada levando-se em 
consideração a precisão dos experimentos (quadrados médios dos resíduos dados na tabela 4) ou seja, experimentos menos precisos têm pesos menores e experimentos mais precisos têm pesos maiores na análise ponderada. 


\section{CONCLUSÕES}

Através dos resultados obtidos, pode-se concluir que:

1. As interações $E \times N^{\prime}, E \times N^{\prime \prime}, E \times P^{\prime}, E \times P^{\prime \prime}, E \times K^{\prime}$ e $E \times K^{\prime \prime}$, conforme demonstrado, são os resíduos específicos para se testar os efeitos de N', N', P', P', $K^{\prime}$ e $K^{\prime \prime}$, respectivamente. Da mesma forma, as interações $E \times N^{\prime} \times P^{\prime}, E \times N^{\prime} \times K^{\prime}$ e $E \times P^{\prime} \times K^{\prime}$, são os resíduos específicos para se testar os efeitos de $N^{\prime} \times P^{\prime}, N^{\prime} \times K^{\prime}$ e $\mathrm{P}^{\prime} \times \mathrm{K}^{\prime}$, respectivamente.

2. O ajuste dos números de graus de liberdade, pelo método de Cochran, não produziu diferenças significativas nos resultados da análise de variância não ponderada. A ocorrência deste fato se deve em função do elevado número de graus de liberdade para o resíduo médio e para as interações $\mathrm{E} \times \mathrm{N}, \mathrm{E} \times \mathrm{P}$ e $\mathrm{E} \times \mathrm{K}$.

3. Para as interações experimentos com tratamentos, é preciso investigar se os contrastes que compõem estas interações possuem quadrados médios homogêneos. Caso não sejam homogêneos, é preciso testar os contrastes de interesse com os respectivos resíduos específicos.

4. Os resultados obtidos com o uso do teste de combinação de probabilidades foram, praticamente, os mesmos obtidos através do teste $F$, na análise de variância não ponderada. Uma pequena diferença verificada em um dos testes é natural, pois, tratase de testes com metodologias diferentes. Quando existe interação significativa, 
como na análise ponderada, não convém usar o teste de combinação de probabilidades, pois, este teste não considera a existência de interações.

5. A análise de variância ponderada mostrou ser mais eficiente para o caso de heterogeneidade de variâncias entre os experimentos. As diferenças entre esta análise e a não ponderada são devidas a heterogeneidade de variâncias entre os experimentos, não controlada na análise não ponderada, pois o ajuste dos números de graus de liberdade, pelo método de Cochran (1954), não se mostrou eficiente.

6. Verificou-se a necessidade do uso do resíduo específico, também, na análise ponderada, pois, esta análise controla somente a heterogeneidade de variâncias entre os experimentos e não a heterogeneidade de variâncias das interações.

7. Em resumo, pode-se indicar uma análise de variância ponderada para o caso de heterogeneidade de variâncias entre os experimentos, o uso do resíduo específico no caso de heterogeneidade das variâncias das interações, independente de que análise se está usando e o uso do teste de combinação de probabilidades para se testar os contrastes de interesse após uma análise preliminar, quando as interações não são significativas. 


\section{REFERÊNCIAS BIBLIOGRÁFICAS}

BANZATTO, D. A. ; KRONKA, S. N. Experimentação Agrícola. Jaboticabal: FUNEP-UNESP, 1989. $247 \mathrm{p}$.

BARBIN, D. Componentes de Variância. Piracicaba: FEALQ, 1998. 120 p.

CAMPOS, H. Estatística Aplicada à Experimentação com cana-de-açucar. Piracicaba: FEALQ, 1984. 292 p.

CAMPOS, H. ; BARBIN, D. Decomposição das interações tratamentos $\times$ locais e tratamentos $\times$ anos em grupos de experimentos. In: SIMPÓSIO DE ESTATÍSTICA APLICADA À EXPERIMENTAÇÃO AGRONÔMICA, 1. Piracicaba, 1985. Anais. Campinas: Fundação Cargill, 1985. p.197-206.

COCHRAN, W. G. Problems arising in the analysis of a series of similar experiments. Joun. Roy. Stat. Soc. Suppl., v.4, p.102-118, 1937.

COCHRAN, W. G. Some consequences when the assumptions for the analysis of variance are not satisfied. Biometrics. v.3, p.22-38, 1946.

COCHRAN, W. G. The combination of estimates from different experiments. Biometrics. v.10, p.101-129, 1954.

COCHRAN, W. G. ; CARROLL, S. P. A sampling investigation of the efficiency of weighting inversely as the estimated variance. Biometrics, v.9, p.447-459, 1953. 
COCHRAN, W. G. ; COX, G. M. Experimental Designs. 2.ed. New York: John Wiley and Sons, Inc. 1957. $611 \mathrm{p}$.

CONAGIN, A. Teste de significância coletiva. In: SIMPÓSIO DE ESTATÍSTICA APLICADA À EXPERIMENTAÇÃO AGRONÔMICA, 1. Piracicaba, 1985. Anais. Campinas: Fundação Cargill, 1985. p.298-310.

CONAGIN, A. ; IGUE, T. ; NAGUAI, V. Teste de combinação de probabilidades em grupos de experimentos. Boletim Cient. Inst. Agron. Campinas, $n^{\circ}$ 30, p.1-9, 1993.

FERREIRA, L. E. P. A decomposição do resíduo em casos de heterocedasticidade nas análises de variância de ensaios em blocos casualizados. Piracicaba, 1978. 64 p. Dissertação (M.S.) - ESALQ-USP.

FERREIRA, P. V. Estatística Experimental Aplicada à Agronomia. 2.ed. Maceió: EDUFAL, 1996. 604 p.

FISHER, R. A. ; YATES, F. Statistical tables for biological, agricultural and medical research. 3.ed. Edinburgh: Oliver and Boyd, 1948.

JAMES, G. S. The comparison of several groups of observations when the ratios of the population variances are unknown. Biometrika, v.38, p.324-329, 1951.

KEMPTHORNE, $O$. The design and analysis of experiments. New York: John Wiley and Sons, Inc. 1952. $631 \mathrm{p}$.

MALAVOLTA, E. ; PIMENTEL GOMES, F. ; COURY, T. et al. A diagnose foliar na cana-de-açucar IV. Resultados de 40 ensaios fatoriais NPK $3 \times 3 \times 3$, primeiro corte no estado de São Paulo. ESALQ-USP, Piracicaba, 1963. 47 p. 
MEIER, P. Variance of a weighted mean. Biometrics, v.9, p.59-73, 1953.

MILliKEN, G. A. ; JOHNSON, D. E. Analysis of Messy Data. v.1, New York: Chapman \&Hall, 1992. 473 p.

PAULL, A. E. On a preliminary test for pooling mean squares in the analysis of variance. Ann. Math. Statist., v.21, p.539-556, 1950.

PIMENTEL GOMES, F. Curso de Estatística Experimental. 12. ed. Piracicaba: Nobel, 1987. $467 \mathrm{p}$.

PIMENTEL GOMES, F. ; SALATI, E. As interações num grupo de experimentos de adubação de cana de açúcar. Revista de Agricultura, v.32, p.261-270, 1957.

SATTERTHWAITE, F. E. An approximate distribution of estimates of variance components. Biometrics Bulletin, v.2, p. 110-114, 1946.

SNEDECOR, G. W. ; COCHRAN, W. G. Statistical Methods. 2.ed. Iowa: The Iowa State University Press, 1967. 593 p.

WELCH, B. L. On the comparison of several mean values: an alternative approach. Biometrika, v.38, p.330-336, 1951.

YATES, F. The design and analysis of factorial experiments. Imp. Bur. Soil Sci. Tech. Comm., v.35, 1937.

YATES, F. ; COCHRAN, W. G. The analysis of groups of experiments. Joun. Agr. Sci., v.28, p.556-580, 1938. 


\section{APÊNDICE 1}

Rendimentos de cana-de-açúcar por experimento 
Tabela 12. Produção de cana-planta (t/ha) em terra roxa legítima.

\begin{tabular}{|c|c|c|c|c|c|c|}
\hline Tratamentos & & & Exper & & & \\
\hline N P K & 1 & 2 & 3 & 4 & 5 & 6 \\
\hline 000 & 130,5 & 113,9 & 65,7 & 43,4 & 265,2 & 221,6 \\
\hline 101 & 148,2 & 127,8 & 88,7 & 72,3 & 273,7 & 260,2 \\
\hline 202 & 120,7 & 153,7 & 114,3 & 107,1 & 285,0 & 255,7 \\
\hline 012 & 142,5 & 133,4 & 107,7 & 88,7 & 233,4 & 283,0 \\
\hline 110 & 143,6 & 141,6 & 75,3 & 78,2 & 225,0 & 241,6 \\
\hline 211 & 146,6 & 145,0 & 98,9 & 110,7 & 261,2 & 293,9 \\
\hline 021 & 146,4 & 132,0 & 90,1 & 72,7 & 244,5 & 266,6 \\
\hline 122 & 156,2 & 146,8 & 104,8 & 96,6 & 239,3 & 292,3 \\
\hline 220 & 134,5 & 133,2 & 110,3 & 66,4 & 256,6 & 251,6 \\
\hline 002 & 122,8 & 131,2 & 98,2 & 96,1 & 269,6 & 280,9 \\
\hline 100 & 129,8 & 139,3 & 61,1 & 56,2 & 257,7 & 298,6 \\
\hline 201 & 137,7 & 162,0 & 89,8 & 98,6 & 283,4 & 300,2 \\
\hline 011 & 146,4 & 140,5 & 88,9 & 88,7 & 273,6 & 270,0 \\
\hline 112 & 136,2 & 145,0 & 105,3 & 115,9 & 274,6 & 252,1 \\
\hline 210 & 126,8 & 147,0 & 61,1 & 63,4 & 237,1 & 243,6 \\
\hline 020 & 118,2 & 115,7 & 67,8 & 68,7 & 288,9 & 236,6 \\
\hline 121 & 130,7 & 151,8 & 101,6 & 101,6 & 239,3 & 246,2 \\
\hline 222 & 133,7 & 153,4 & 121,4 & 116,4 & 254,1 & 289,8 \\
\hline 001 & 117,0 & 127,5 & 91,2 & 95,5 & 246,1 & 233,0 \\
\hline 102 & 149,5 & 133,4 & 104,8 & 113,4 & 260,5 & 266,0 \\
\hline 200 & 124,5 & 142,7 & 76,1 & 74,2 & 263,6 & 235,2 \\
\hline 010 & 135,2 & 120,0 & 65,7 & 92,3 & 273,9 & 239,8 \\
\hline 111 & 131,4 & 144,5 & 120,9 & 91,6 & 237,1 & 230,5 \\
\hline 212 & 138,0 & 166,8 & 111,8 & 116,6 & 271,4 & 273,9 \\
\hline 022 & 113,9 & 114,5 & 95,9 & 122,8 & 281,8 & 296,2 \\
\hline 120 & 109,3 & 131,2 & 72,3 & 71,4 & 265,0 & 227,5 \\
\hline 221 & 139,3 & 139,5 & 100,1 & 111,2 & 280,2 & 273,9 \\
\hline
\end{tabular}


Tabela 12. Produção de cana-planta (t/ha) em terra roxa legítima (continuação).

\begin{tabular}{|c|c|c|c|c|c|}
\hline \multirow{2}{*}{$\begin{array}{l}\text { Tratamentos } \\
\text { NP K }\end{array}$} & \multicolumn{5}{|c|}{ Experimentos } \\
\hline & 7 & 8 & 9 & 10 & 11 \\
\hline 000 & 252,0 & 70,5 & 220,2 & 95,0 & 148,9 \\
\hline 101 & 269,0 & 106,2 & 256,8 & 150,7 & 187,8 \\
\hline 202 & 256,4 & 110,0 & 212,7 & 141,1 & 195,9 \\
\hline 012 & 267,7 & 131,2 & 226,1 & 150,7 & 193,7 \\
\hline 110 & 255,0 & 118,9 & 296,6 & 130,9 & 164,3 \\
\hline 211 & 256,8 & 115,2 & 233,9 & 150,5 & 174,6 \\
\hline 021 & 239,3 & 101,6 & 254,5 & 170,3 & 183,0 \\
\hline 122 & 254,5 & 124,8 & 220,3 & 142,8 & 169,5 \\
\hline 220 & 264,0 & 90,3 & 216,8 & 144,6 & 173,7 \\
\hline 002 & 282,7 & 109,5 & 176,2 & 146,1 & 165,9 \\
\hline 100 & 218,4 & 129,5 & 228,6 & 144,5 & 188,9 \\
\hline 201 & 264,8 & 113,2 & 221,8 & 173,6 & 156,4 \\
\hline 011 & 252,1 & 96,6 & 175,9 & 167,5 & 185,7 \\
\hline 112 & 272,5 & 112,7 & 224,8 & 133,7 & 162,5 \\
\hline 210 & 248,4 & 134,6 & 235,9 & 134,5 & 137,1 \\
\hline 020 & 202,1 & 101,8 & 228,7 & 130,9 & 148,7 \\
\hline 121 & 249,5 & 130,5 & 220,9 & 175,2 & 163,3 \\
\hline 222 & 276,8 & 152,0 & 230,5 & 153,2 & 194,3 \\
\hline 001 & 236,4 & 117,8 & 202,7 & 138,2 & 163,1 \\
\hline 102 & 251,4 & 137,0 & 228,2 & 169,8 & 194,5 \\
\hline 200 & 264,3 & 120,9 & 162,3 & 144,5 & 153,2 \\
\hline 010 & 245,7 & 147,0 & 160,5 & 152,5 & 215,6 \\
\hline 111 & 248,9 & 105,2 & 219,3 & 125,7 & 155,6 \\
\hline 212 & 243,0 & 128,7 & 230,9 & 145,5 & 155,6 \\
\hline 022 & 254,5 & 122,7 & 191,2 & 157,3 & 169,9 \\
\hline 120 & 257,0 & 139,1 & 254,6 & 158,4 & 172,0 \\
\hline 221 & 257,0 & 127,7 & 207,5 & 146,6 & 197,7 \\
\hline
\end{tabular}




\section{APÊNDICE 2}

Programa SAS para análise de variância (não ponderada) 


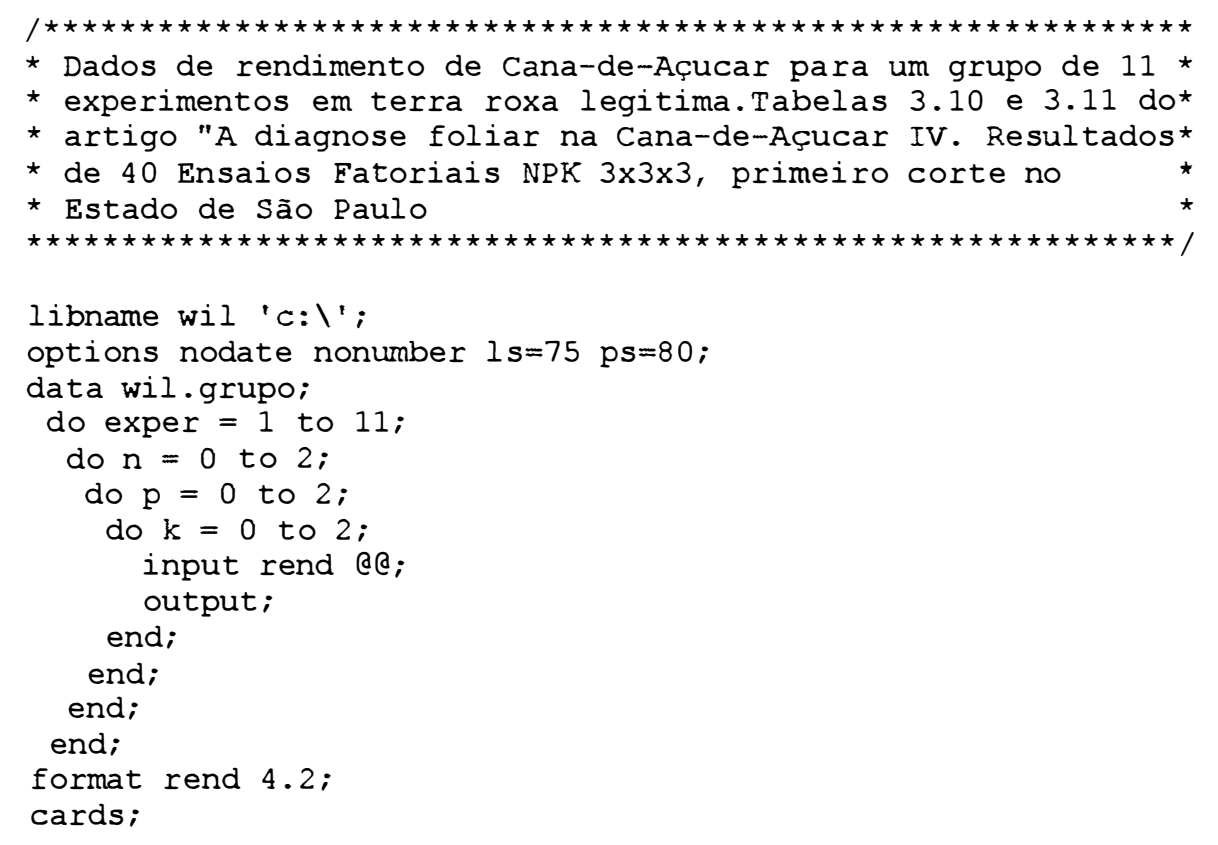

$\begin{array}{lllllllll}130.5 & 117.0 & 122.8 & 135.2 & 146.4 & 142.5 & 118.2 & 146.4 & 113.9\end{array}$ $\begin{array}{lllllllll}129.8 & 148.2 & 149.5 & 143.6 & 131.4 & 136.2 & 109.3 & 130.7 & 156.2\end{array}$ $\begin{array}{llllllllll}124.5 & 137.7 & 120.7 & 126.8 & 146.6 & 138.0 & 134.5 & 139.3 & 133.7\end{array}$ $\begin{array}{lllllllll}113.9 & 127.5 & 131.2 & 120.0 & 140.5 & 133.4 & 115.7 & 132.0 & 114.5\end{array}$ $\begin{array}{lllllllll}139.3 & 127.8 & 133.4 & 141.6 & 144.5 & 145.0 & 131.2 & 151.8 & 146.8\end{array}$ $\begin{array}{llllllllll}142.7 & 162.0 & 153.7 & 147.0 & 145.0 & 166.8 & 133.2 & 139.5 & 153.4\end{array}$ $\begin{array}{lllllllll}65.7 & 91.2 & 98.2 & 65.7 & 88.9 & 107.7 & 67.8 & 90.1 & 95.9\end{array}$ $\begin{array}{lllllllll}61.1 & 88.7 & 104.8 & 75.3 & 120.9 & 105.3 & 72.3 & 101.6 & 104.8\end{array}$ $\begin{array}{lllllllll}76.1 & 89.8 & 114.3 & 61.1 & 98.9 & 111.8 & 110.3 & 100.1 & 121.4\end{array}$

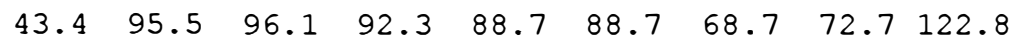
$\begin{array}{lllllllll}56.2 & 72.3 & 113.4 & 78.2 & 91.6 & 115.9 & 71.4 & 101.6 & 96.6\end{array}$ $\begin{array}{lllllllll}74.2 & 98.6 & 107.1 & 63.4 & 110.7 & 116.6 & 66.4 & 111.2 & 116.4\end{array}$ $\begin{array}{lllllllll}265.2 & 246.1 & 269.6 & 273.9 & 273.6 & 233.4 & 288.9 & 244.5 & 281.8\end{array}$ $\begin{array}{llllllllll}257.7 & 273.7 & 260.5 & 225.0 & 237.1 & 274.6 & 265.0 & 239.3 & 239.3\end{array}$ $\begin{array}{lllllllll}263.6 & 283.4 & 285.0 & 237.1 & 261.2 & 271.4 & 256.6 & 280.2 & 254.1\end{array}$ $\begin{array}{lllllllll}221.6 & 233.0 & 280.9 & 239.8 & 270.0 & 283.0 & 236.6 & 266.6 & 296.2\end{array}$ $\begin{array}{lllllllll}298.6 & 260.2 & 266.0 & 241.6 & 230.5 & 252.1 & 227.5 & 246.2 & 292.3\end{array}$ $\begin{array}{lllllllll}235.2 & 300.2 & 255.7 & 243.6 & 293.9 & 273.9 & 251.6 & 273.9 & 289.8\end{array}$ $\begin{array}{llllllllll}252.0 & 236.4 & 282.7 & 245.7 & 252.1 & 267.7 & 202.1 & 239.3 & 254.5\end{array}$ $\begin{array}{lllllllll}218.4 & 269.0 & 251.4 & 255.0 & 248.9 & 272.5 & 257.0 & 249.5 & 254.5\end{array}$ $\begin{array}{lllllllll}264.3 & 264.8 & 256.4 & 248.4 & 256.8 & 243.0 & 264.0 & 257.0 & 276.8\end{array}$ $\begin{array}{lllllllll}70.5 & 117.8 & 109.5 & 147.0 & 96.6 & 131.2 & 101.8 & 101.6 & 122.7\end{array}$

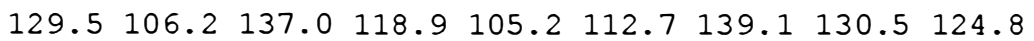
$\begin{array}{lllllllll}120.9 & 113.2 & 110.0 & 134.6 & 115.2 & 128.7 & 90.3 & 127.7 & 152.0\end{array}$ $\begin{array}{lllllllll}220.2 & 202.7 & 176.2 & 160.5 & 175.9 & 226.1 & 228.7 & 254.5 & 191.2\end{array}$ $\begin{array}{lllllllll}228.6 & 256.8 & 228.2 & 269.6 & 219.3 & 224.8 & 254.6 & 220.9 & 220.3\end{array}$ $\begin{array}{llllllllll}162.3 & 221.8 & 212.7 & 235.9 & 233.9 & 230.9 & 216.8 & 207.5 & 230.5\end{array}$ $\begin{array}{lllllllll}95.0 & 138.2 & 146.1 & 152.5 & 167.5 & 150.7 & 130.9 & 170.3 & 157.3\end{array}$ $\begin{array}{llllllllll}144.5 & 150.7 & 169.8 & 130.9 & 125.7 & 133.7 & 158.4 & 175.2 & 142.8\end{array}$ $\begin{array}{lllllllll}144.5 & 173.6 & 141.1 & 134.5 & 150.5 & 145.5 & 144.6 & 146.6 & 153.2\end{array}$ $\begin{array}{llllllllll}148.9 & 163.1 & 165.9 & 215.6 & 185.7 & 193.7 & 148.7 & 183.0 & 169.9\end{array}$ $\begin{array}{lllllllll}188.9 & 187.8 & 194.5 & 164.3 & 155.6 & 162.5 & 172.0 & 163.3 & 169.5\end{array}$ $\begin{array}{llllllllll}153.2 & 156.4 & 195.9 & 137.1 & 174.6 & 155.6 & 173.7 & 197.7 & 194.3\end{array}$ ;

run; 


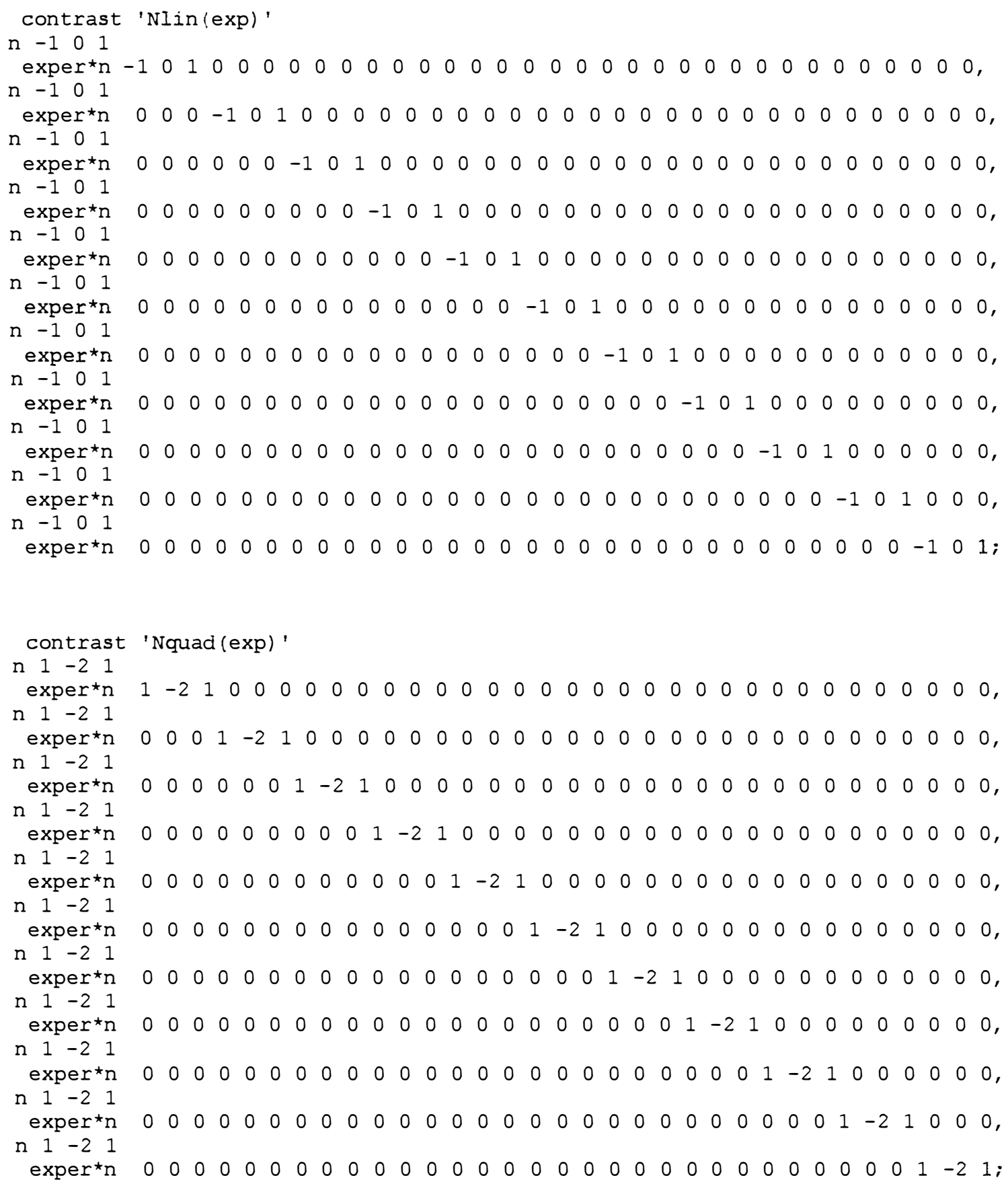




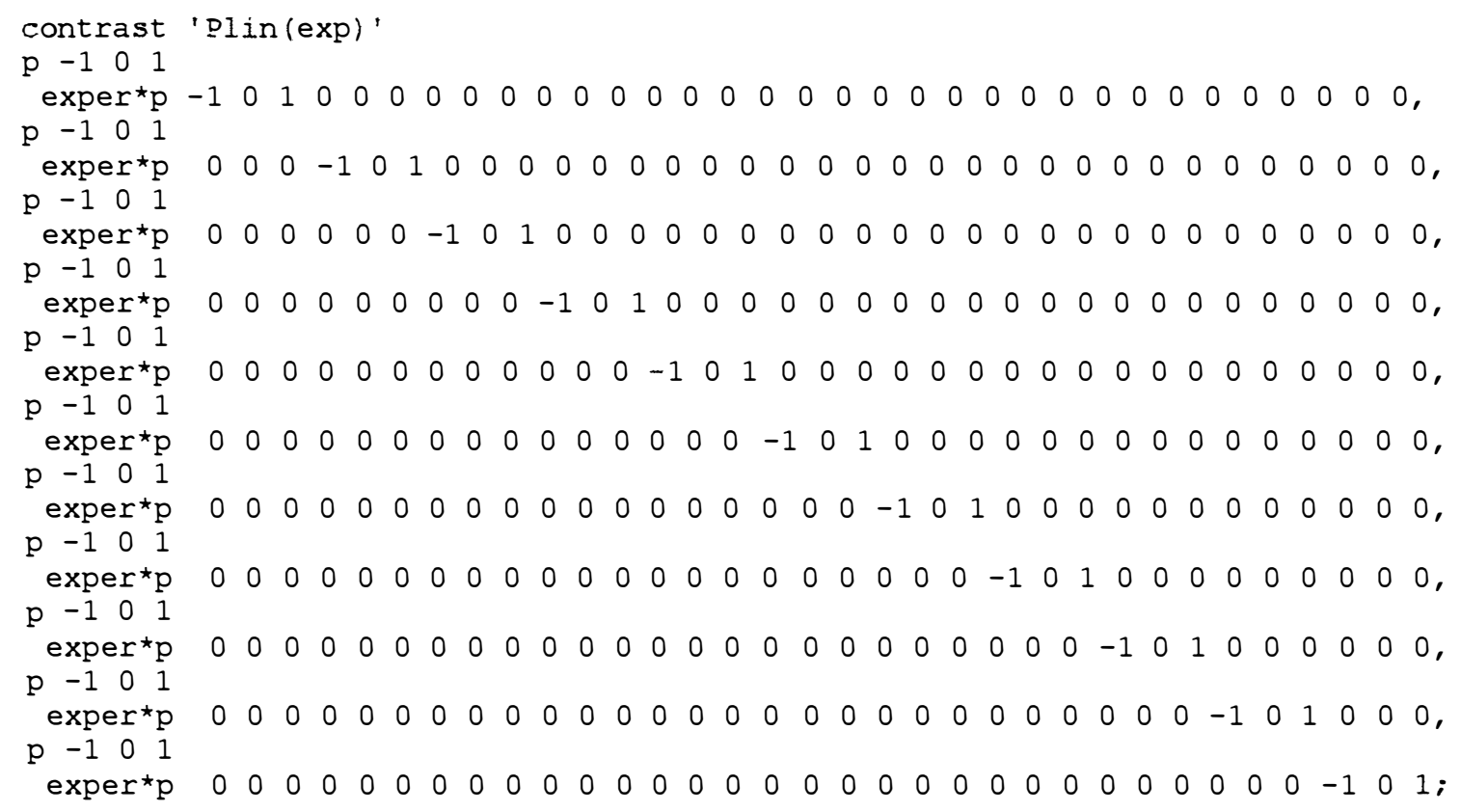




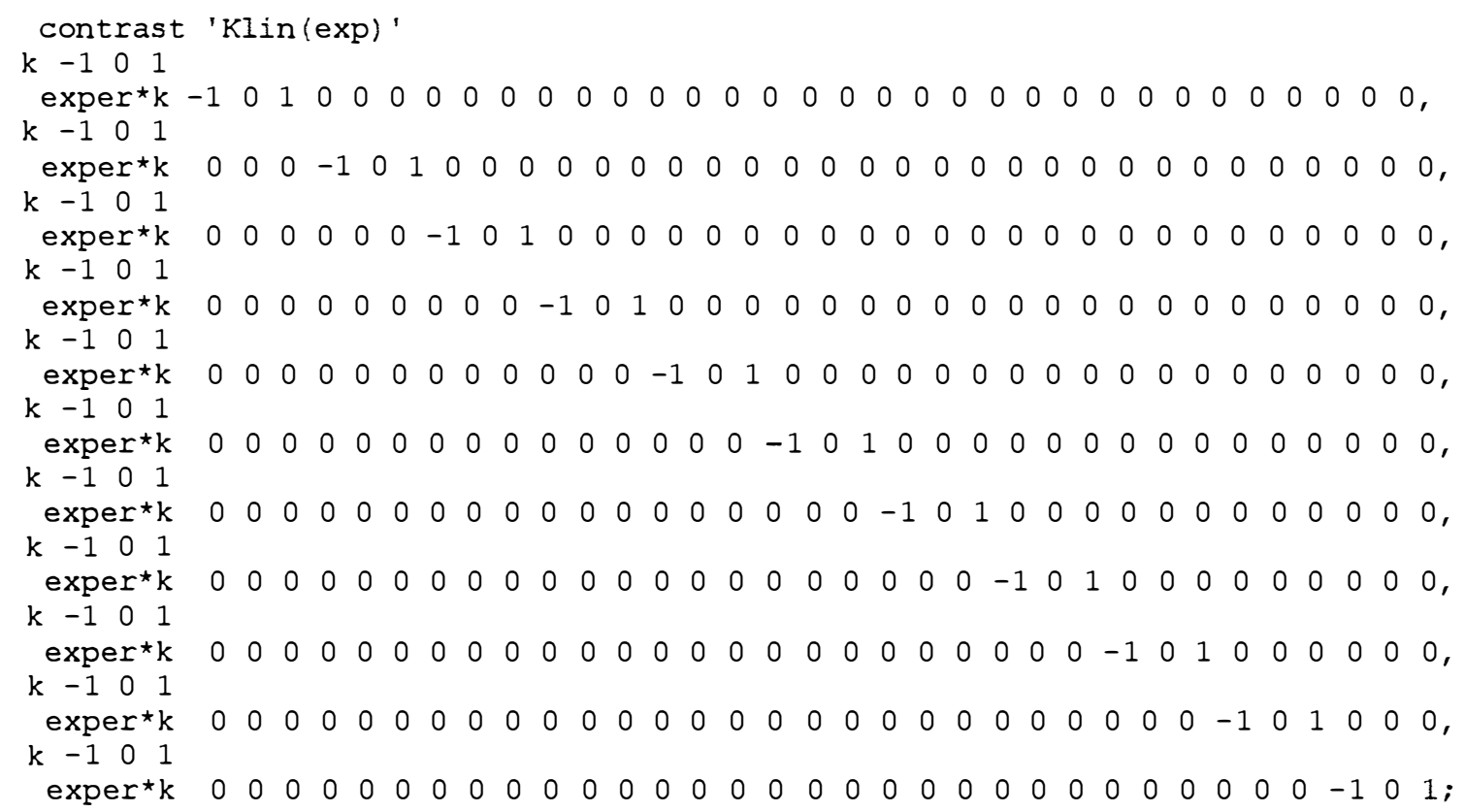




\begin{tabular}{|c|c|c|c|c|c|c|c|c|c|}
\hline \multicolumn{10}{|c|}{ contrast 'NlinxPlinx (exp)' } \\
\hline$n * p \quad 1 \quad 0$ & -1 & 0 & 0 & 0 & -1 & 0 & 1 & & \\
\hline \multirow[t]{11}{*}{ exper* $n^{\star} p$} & 1 & 0 & -1 & 0 & 0 & 0 & -1 & 0 & 1 \\
\hline & 0 & 0 & 0 & 0 & 0 & 0 & 0 & 0 & 0 \\
\hline & 0 & 0 & 0 & 0 & 0 & 0 & 0 & 0 & 0 \\
\hline & 0 & 0 & 0 & 0 & 0 & 0 & 0 & 0 & 0 \\
\hline & 0 & 0 & 0 & 0 & 0 & 0 & 0 & 0 & 0 \\
\hline & 0 & 0 & 0 & 0 & 0 & 0 & 0 & 0 & 0 \\
\hline & 0 & 0 & 0 & 0 & 0 & 0 & 0 & 0 & 0 \\
\hline & 0 & 0 & 0 & 0 & 0 & 0 & 0 & 0 & 0 \\
\hline & 0 & 0 & 0 & 0 & 0 & 0 & 0 & 0 & 0 \\
\hline & 0 & 0 & 0 & 0 & 0 & 0 & 0 & 0 & 0 \\
\hline & 0 & 0 & 0 & 0 & 0 & 0 & 0 & 0 & \\
\hline
\end{tabular}

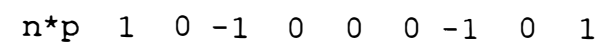

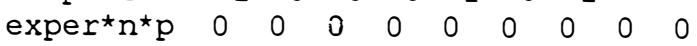
$\begin{array}{rrrrrrrrr}1 & 0 & -1 & 0 & 0 & 0 & -1 & 0 & 1 \\ 0 & 0 & 0 & 0 & 0 & 0 & 0 & 0 & 0\end{array}$ $\begin{array}{lllllllll}0 & 0 & 0 & 0 & 0 & 0 & 0 & 0 & 0\end{array}$ $\begin{array}{lllllllll}0 & 0 & 0 & 0 & 0 & 0 & 0 & 0 & 0\end{array}$ $\begin{array}{lllllllll}0 & 0 & 0 & 0 & 0 & 0 & 0 & 0 & 0\end{array}$ $\begin{array}{lllllllll}0 & 0 & 0 & 0 & 0 & 0 & 0 & 0 & 0\end{array}$ $\begin{array}{lllllllll}0 & 0 & 0 & 0 & 0 & 0 & 0 & 0 & 0\end{array}$ $\begin{array}{lllllllll}0 & 0 & 0 & 0 & 0 & 0 & 0 & 0 & 0\end{array}$ $\begin{array}{lllllllll}0 & 0 & 0 & 0 & 0 & 0 & 0 & 0 & 0 \\ 0 & 0 & 0 & 0 & 0 & 0 & 0 & 0 & 0\end{array}$

\begin{tabular}{|c|c|c|c|c|c|c|c|c|c|}
\hline \multirow{12}{*}{$\begin{array}{l}n^{\star} p \\
\text { expe } r^{\star} n^{\star} p\end{array}$} & \multirow{2}{*}{$\begin{array}{l}-1 \\
0\end{array}$} & \multirow{2}{*}{\multicolumn{2}{|c|}{$\begin{array}{ll}0 & 0 \\
0 & 0\end{array}$}} & \multirow{2}{*}{$0^{0}$} & \multirow{2}{*}{$\begin{array}{l}-1 \\
0\end{array}$} & \multicolumn{2}{|c|}{$\begin{array}{ll}0 & 1\end{array}$} & \\
\hline & & & & & & 0 & 0 & 0 & \\
\hline & 0 & 0 & 0 & 0 & 0 & 0 & 0 & 0 & \\
\hline & 1 & 0 & -1 & 0 & 0 & 0 & -1 & 0 & \\
\hline & 0 & 0 & 0 & 0 & 0 & 0 & 0 & 0 & \\
\hline & 0 & 0 & 0 & 0 & 0 & 0 & 0 & 0 & \\
\hline & 0 & 0 & 0 & 0 & 0 & 0 & 0 & 0 & \\
\hline & 0 & 0 & 0 & 0 & 0 & 0 & 0 & 0 & \\
\hline & 0 & 0 & 0 & 0 & 0 & 0 & 0 & 0 & \\
\hline & 0 & 0 & 0 & 0 & 0 & 0 & 0 & 0 & \\
\hline & 0 & 0 & 0 & 0 & 0 & 0 & 0 & 0 & \\
\hline & 0 & 0 & 0 & 0 & 0 & 0 & 0 & 0 & \\
\hline
\end{tabular}

$\begin{array}{llllllllll}n * p & 1 & 0 & -1 & 0 & 0 & 0 & -1 & 0 & 1\end{array}$

$\begin{array}{rrrrrrrrrr}\text { exper* } n^{\star} p & 0 & 0 & 0 & 0 & 0 & 0 & 0 & 0 & 0 \\ & 0 & 0 & 0 & 0 & 0 & 0 & 0 & 0 & 0 \\ 0 & 0 & 0 & 0 & 0 & 0 & 0 & 0 & 0 \\ 1 & 0 & -1 & 0 & 0 & 0 & -1 & 0 & 1 \\ 0 & 0 & 0 & 0 & 0 & 0 & 0 & 0 & 0 \\ 0 & 0 & 0 & 0 & 0 & 0 & 0 & 0 & 0 \\ 0 & 0 & 0 & 0 & 0 & 0 & 0 & 0 & 0 \\ 0 & 0 & 0 & 0 & 0 & 0 & 0 & 0 & 0 \\ 0 & 0 & 0 & 0 & 0 & 0 & 0 & 0 & 0 \\ 0 & 0 & 0 & 0 & 0 & 0 & 0 & 0 & 0 \\ 0 & 0 & 0 & 0 & 0 & 0 & 0 & 0 & 0,\end{array}$




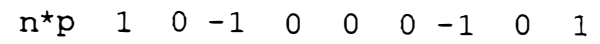

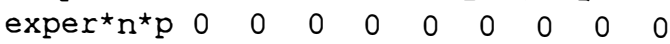
$\begin{array}{lllllllll}0 & 0 & 0 & 0 & 0 & 0 & 0 & 0 & 0\end{array}$ $\begin{array}{lllllllll}0 & 0 & 0 & 0 & 0 & 0 & 0 & 0 & 0\end{array}$ $\begin{array}{lllllllll}0 & 0 & 0 & 0 & 0 & 0 & 0 & 0 & 0\end{array}$ $\begin{array}{lllllllll}1 & 0 & -1 & 0 & 0 & 0 & -1 & 0 & 1\end{array}$ $\begin{array}{lllllllll}0 & 0 & 0 & 0 & 0 & 0 & 0 & 0 & 0\end{array}$ $\begin{array}{lllllllll}0 & 0 & 0 & 0 & 0 & 0 & 0 & 0 & 0\end{array}$ $\begin{array}{lllllllll}0 & 0 & 0 & 0 & 0 & 0 & 0 & 0 & 0\end{array}$ $\begin{array}{lllllllll}0 & 0 & 0 & 0 & 0 & 0 & 0 & 0 & 0\end{array}$ $\begin{array}{lllllllll}0 & 0 & 0 & 0 & 0 & 0 & 0 & 0 & 0 \\ 0 & 0 & 0 & 0 & 0 & 0 & 0 & 0 & 0\end{array}$

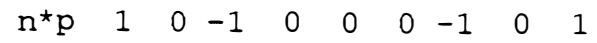

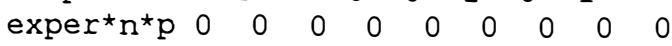
$\begin{array}{lllllllll}0 & 0 & 0 & 0 & 0 & 0 & 0 & 0 & 0 \\ 0 & 0 & 0 & 0 & 0 & 0 & 0 & 0 & 0\end{array}$ $\begin{array}{lllllllll}0 & 0 & 0 & 0 & 0 & 0 & 0 & 0 & 0\end{array}$ $\begin{array}{lllllllll}0 & 0 & 0 & 0 & 0 & 0 & 0 & 0 & 0\end{array}$ $\begin{array}{lllllllll}1 & 0 & -1 & 0 & 0 & 0 & -1 & 0 & 1\end{array}$ $\begin{array}{lllllllll}0 & 0 & 0 & 0 & 0 & 0 & 0 & 0 & 0\end{array}$ $\begin{array}{lllllllll}0 & 0 & 0 & 0 & 0 & 0 & 0 & 0 & 0\end{array}$ $\begin{array}{lllllllll}0 & 0 & 0 & 0 & 0 & 0 & 0 & 0 & 0\end{array}$ $\begin{array}{lllllllll}0 & 0 & 0 & 0 & 0 & 0 & 0 & 0 & 0 \\ 0 & 0 & 0 & 0 & 0 & 0 & 0 & 0 & 0,\end{array}$

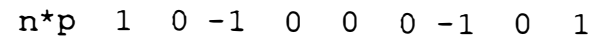

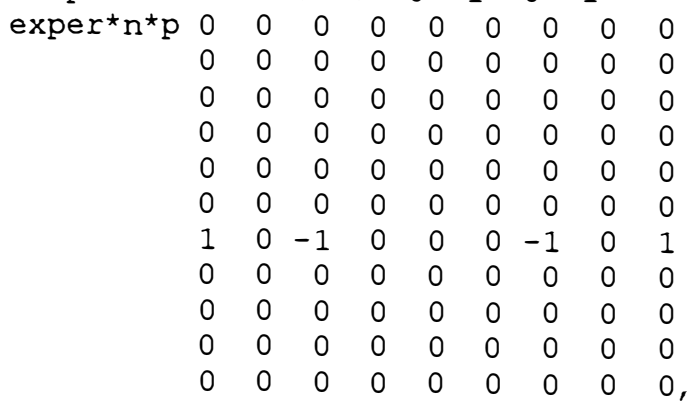

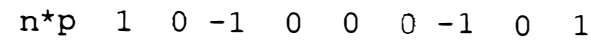

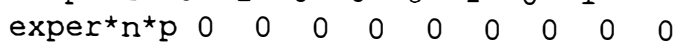
$\begin{array}{lllllllll}0 & 0 & 0 & 0 & 0 & 0 & 0 & 0 & 0\end{array}$ $\begin{array}{lllllllll}0 & 0 & 0 & 0 & 0 & 0 & 0 & 0 & 0\end{array}$ $\begin{array}{lllllllll}0 & 0 & 0 & 0 & 0 & 0 & 0 & 0 & 0\end{array}$ $\begin{array}{lllllllll}0 & 0 & 0 & 0 & 0 & 0 & 0 & 0 & 0\end{array}$ $\begin{array}{lllllllll}0 & 0 & 0 & 0 & 0 & 0 & 0 & 0 & 0\end{array}$ $\begin{array}{lllllllll}0 & 0 & 0 & 0 & 0 & 0 & 0 & 0 & 0\end{array}$ $\begin{array}{lllllllll}1 & 0 & -1 & 0 & 0 & 0 & -1 & 0 & 1\end{array}$ $\begin{array}{lllllllll}0 & 0 & 0 & 0 & 0 & 0 & 0 & 0 & 0\end{array}$ $\begin{array}{lllllllll}0 & 0 & 0 & 0 & 0 & 0 & 0 & 0 & 0 \\ 0 & 0 & 0 & 0 & 0 & 0 & 0 & 0 & 0\end{array}$ 


$\begin{array}{rrrrrrrrrr}\mathrm{n}^{\star} \mathrm{p} & 1 & 0 & -1 & 0 & 0 & 0 & -1 & 0 & 1 \\ \text { exper* } \mathrm{n}^{\star} \mathrm{p} & 0 & 0 & 0 & 0 & 0 & 0 & 0 & 0 & 0 \\ 0 & 0 & 0 & 0 & 0 & 0 & 0 & 0 & 0 \\ 0 & 0 & 0 & 0 & 0 & 0 & 0 & 0 & 0 \\ 0 & 0 & 0 & 0 & 0 & 0 & 0 & 0 & 0 \\ 0 & 0 & 0 & 0 & 0 & 0 & 0 & 0 & 0 \\ 0 & 0 & 0 & 0 & 0 & 0 & 0 & 0 & 0 \\ 0 & 0 & 0 & 0 & 0 & 0 & 0 & 0 & 0 \\ 0 & 0 & 0 & 0 & 0 & 0 & 0 & 0 & 0 \\ 1 & 0 & -1 & 0 & 0 & 0 & -1 & 0 & 1 \\ 0 & 0 & 0 & 0 & 0 & 0 & 0 & 0 & 0 \\ 0 & 0 & 0 & 0 & 0 & 0 & 0 & 0 & 0,\end{array}$

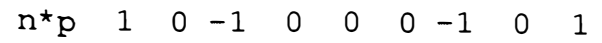

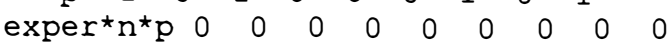
$\begin{array}{lllllllll}0 & 0 & 0 & 0 & 0 & 0 & 0 & 0 & 0 \\ 0 & 0 & 0 & 0 & 0 & 0 & 0 & 0 & 0\end{array}$ $\begin{array}{lllllllll}0 & 0 & 0 & 0 & 0 & 0 & 0 & 0 & 0\end{array}$ $\begin{array}{lllllllll}0 & 0 & 0 & 0 & 0 & 0 & 0 & 0 & 0\end{array}$ $\begin{array}{lllllllll}0 & 0 & 0 & 0 & 0 & 0 & 0 & 0 & 0\end{array}$ $\begin{array}{lllllllll}0 & 0 & 0 & 0 & 0 & 0 & 0 & 0 & 0\end{array}$ $\begin{array}{lllllllll}0 & 0 & 0 & 0 & 0 & 0 & 0 & 0 & 0\end{array}$ $\begin{array}{lllllllll}0 & 0 & 0 & 0 & 0 & 0 & 0 & 0 & 0\end{array}$ $\begin{array}{rrrrrrrrr}1 & 0 & -1 & 0 & 0 & 0 & -1 & 0 & 1 \\ 0 & 0 & 0 & 0 & 0 & 0 & 0 & 0 & 0\end{array}$

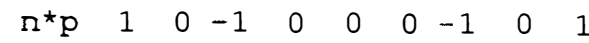
$\begin{array}{rrrrrrrrrr}\text { exper*}{ }^{\star}{ }^{*} \mathrm{p} & 0 & 0 & 0 & 0 & 0 & 0 & 0 & 0 & 0 \\ 0 & 0 & 0 & 0 & 0 & 0 & 0 & 0 & 0 \\ 0 & 0 & 0 & 0 & 0 & 0 & 0 & 0 & 0 \\ 0 & 0 & 0 & 0 & 0 & 0 & 0 & 0 & 0 \\ 0 & 0 & 0 & 0 & 0 & 0 & 0 & 0 & 0 \\ 0 & 0 & 0 & 0 & 0 & 0 & 0 & 0 & 0 \\ 0 & 0 & 0 & 0 & 0 & 0 & 0 & 0 & 0 \\ 0 & 0 & 0 & 0 & 0 & 0 & 0 & 0 & 0 \\ 0 & 0 & 0 & 0 & 0 & 0 & 0 & 0 & 0 \\ 0 & 0 & 0 & 0 & 0 & 0 & 0 & 0 & 0 \\ 1 & 0 & -1 & 0 & 0 & 0 & -1 & 0 & 1 ;\end{array}$

contrast 'NlinxKlinx (exp)'

$n n^{\star} k \begin{array}{lllllllll}1 & 0 & -1 & 0 & 0 & 0 & -1 & 0 & 1\end{array}$

exper* $n^{\star} k \begin{array}{lllllllll}1 & 0 & -1 & 0 & 0 & 0 & -1 & 0 & 1\end{array}$

$\begin{array}{lllllllll}0 & 0 & 0 & 0 & 0 & 0 & 0 & 0 & 0\end{array}$

$\begin{array}{lllllllll}0 & 0 & 0 & 0 & 0 & 0 & 0 & 0 & 0\end{array}$

$\begin{array}{lllllllll}0 & 0 & 0 & 0 & 0 & 0 & 0 & 0 & 0\end{array}$

$\begin{array}{lllllllll}0 & 0 & 0 & 0 & 0 & 0 & 0 & 0 & 0\end{array}$

$\begin{array}{lllllllll}0 & 0 & 0 & 0 & 0 & 0 & 0 & 0 & 0\end{array}$

$\begin{array}{lllllllll}0 & 0 & 0 & 0 & 0 & 0 & 0 & 0 & 0\end{array}$

$\begin{array}{lllllllll}0 & 0 & 0 & 0 & 0 & 0 & 0 & 0 & 0\end{array}$

$\begin{array}{lllllllll}0 & 0 & 0 & 0 & 0 & 0 & 0 & 0 & 0\end{array}$

$\begin{array}{lllllllll}0 & 0 & 0 & 0 & 0 & 0 & 0 & 0 & 0 \\ 0 & 0 & 0 & 0 & 0 & 0 & 0 & 0 & 0\end{array}$ 


$\begin{array}{rrrrrrrrrr}\mathrm{n}^{\star} \mathrm{k} 1 & 0 & -1 & 0 & 0 & 0 & -1 & 0 & 1 & \\ \text { exper* } \mathrm{n}^{\star} \mathrm{k} & 0 & 0 & 0 & 0 & 0 & 0 & 0 & 0 & 0 \\ & 1 & 0 & -1 & 0 & 0 & 0 & -1 & 0 & 1 \\ 0 & 0 & 0 & 0 & 0 & 0 & 0 & 0 & 0 \\ 0 & 0 & 0 & 0 & 0 & 0 & 0 & 0 & 0 \\ 0 & 0 & 0 & 0 & 0 & 0 & 0 & 0 & 0 \\ 0 & 0 & 0 & 0 & 0 & 0 & 0 & 0 & 0 \\ 0 & 0 & 0 & 0 & 0 & 0 & 0 & 0 & 0 \\ 0 & 0 & 0 & 0 & 0 & 0 & 0 & 0 & 0 \\ 0 & 0 & 0 & 0 & 0 & 0 & 0 & 0 & 0 \\ 0 & 0 & 0 & 0 & 0 & 0 & 0 & 0 & 0 \\ 0 & 0 & 0 & 0 & 0 & 0 & 0 & 0 & 0,\end{array}$

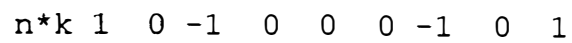

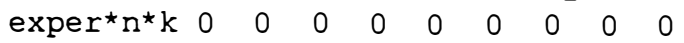
$\begin{array}{lllllllll}0 & 0 & 0 & 0 & 0 & 0 & 0 & 0 & 0\end{array}$ $\begin{array}{lllllllll}1 & 0 & -1 & 0 & 0 & 0 & -1 & 0 & 1\end{array}$

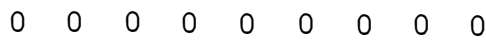
$\begin{array}{lllllllll}0 & 0 & 0 & 0 & 0 & 0 & 0 & 0 & 0\end{array}$ $\begin{array}{lllllllll}0 & 0 & 0 & 0 & 0 & 0 & 0 & 0 & 0\end{array}$ $0 \begin{array}{lllllllll}0 & 0 & 0 & 0 & 0 & 0 & 0 & 0 & 0\end{array}$ $\begin{array}{lllllllll}0 & 0 & 0 & 0 & 0 & 0 & 0 & 0 & 0\end{array}$ $\begin{array}{lllllllll}0 & 0 & 0 & 0 & 0 & 0 & 0 & 0 & 0\end{array}$ $\begin{array}{lllllllll}0 & 0 & 0 & 0 & 0 & 0 & 0 & 0 & 0 \\ 0 & 0 & 0 & 0 & 0 & 0 & 0 & 0 & 0\end{array}$

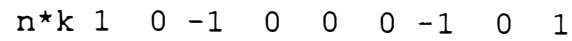

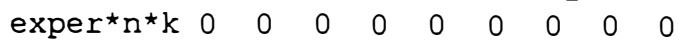

$\begin{array}{rrrrrrrrr}0 & 0 & 0 & 0 & 0 & 0 & 0 & 0 & 0 \\ 0 & 0 & 0 & 0 & 0 & 0 & 0 & 0 & 0 \\ 1 & 0 & -1 & 0 & 0 & 0 & -1 & 0 & 1 \\ 0 & 0 & 0 & 0 & 0 & 0 & 0 & 0 & 0 \\ 0 & 0 & 0 & 0 & 0 & 0 & 0 & 0 & 0 \\ 0 & 0 & 0 & 0 & 0 & 0 & 0 & 0 & 0 \\ 0 & 0 & 0 & 0 & 0 & 0 & 0 & 0 & 0 \\ 0 & 0 & 0 & 0 & 0 & 0 & 0 & 0 & 0 \\ 0 & 0 & 0 & 0 & 0 & 0 & 0 & 0 & 0 \\ 0 & 0 & 0 & 0 & 0 & 0 & 0 & 0 & 0,\end{array}$

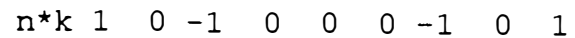
$\begin{array}{llllllllll}\text { exper } r^{\star} n^{\star} k & 0 & 0 & 0 & 0 & 0 & 0 & 0 & 0 & 0\end{array}$ $\begin{array}{lllllllll}0 & 0 & 0 & 0 & 0 & 0 & 0 & 0 & 0\end{array}$ $\begin{array}{lllllllll}0 & 0 & 0 & 0 & 0 & 0 & 0 & 0 & 0\end{array}$ $\begin{array}{lllllllll}0 & 0 & 0 & 0 & 0 & 0 & 0 & 0 & 0\end{array}$ $\begin{array}{lllllllll}1 & 0 & -1 & 0 & 0 & 0 & -1 & 0 & 1\end{array}$ $\begin{array}{lllllllll}0 & 0 & 0 & 0 & 0 & 0 & 0 & 0 & 0\end{array}$ $\begin{array}{lllllllll}0 & 0 & 0 & 0 & 0 & 0 & 0 & 0 & 0\end{array}$ $\begin{array}{lllllllll}0 & 0 & 0 & 0 & 0 & 0 & 0 & 0 & 0\end{array}$ $\begin{array}{lllllllll}0 & 0 & 0 & 0 & 0 & 0 & 0 & 0 & 0\end{array}$ $\begin{array}{lllllllll}0 & 0 & 0 & 0 & 0 & 0 & 0 & 0 & 0 \\ 0 & 0 & 0 & 0 & 0 & 0 & 0 & 0 & 0\end{array}$ 


$\begin{array}{rrrrrrrrrr}\mathrm{n}^{*} \mathrm{k} 1 & 0 & -1 & 0 & 0 & 0 & -1 & 0 & 1 & \\ \text { exper* } \mathrm{n}^{\star} \mathrm{k} & 0 & 0 & 0 & 0 & 0 & 0 & 0 & 0 & 0 \\ 0 & 0 & 0 & 0 & 0 & 0 & 0 & 0 & 0 \\ 0 & 0 & 0 & 0 & 0 & 0 & 0 & 0 & 0 \\ 0 & 0 & 0 & 0 & 0 & 0 & 0 & 0 & 0 \\ 0 & 0 & 0 & 0 & 0 & 0 & 0 & 0 & 0 \\ 1 & 0 & -1 & 0 & 0 & 0 & -1 & 0 & 1 \\ 0 & 0 & 0 & 0 & 0 & 0 & 0 & 0 & 0 \\ 0 & 0 & 0 & 0 & 0 & 0 & 0 & 0 & 0 \\ 0 & 0 & 0 & 0 & 0 & 0 & 0 & 0 & 0 \\ 0 & 0 & 0 & 0 & 0 & 0 & 0 & 0 & 0 \\ 0 & 0 & 0 & 0 & 0 & 0 & 0 & 0 & 0,\end{array}$

$\begin{array}{rrrrrrrrrr}\mathrm{n}^{\star} \mathrm{k} 1 & 0 & -1 & 0 & 0 & 0 & -1 & 0 & 1 & \\ \text { exper* }{ }^{\star} \mathrm{k} & 0 & 0 & 0 & 0 & 0 & 0 & 0 & 0 & 0 \\ 0 & 0 & 0 & 0 & 0 & 0 & 0 & 0 & 0 \\ & 0 & 0 & 0 & 0 & 0 & 0 & 0 & 0 & 0 \\ 0 & 0 & 0 & 0 & 0 & 0 & 0 & 0 & 0 \\ 0 & 0 & 0 & 0 & 0 & 0 & 0 & 0 & 0 \\ 0 & 0 & 0 & 0 & 0 & 0 & 0 & 0 & 0 \\ 1 & 0 & -1 & 0 & 0 & 0 & -1 & 0 & 1 \\ 0 & 0 & 0 & 0 & 0 & 0 & 0 & 0 & 0 \\ 0 & 0 & 0 & 0 & 0 & 0 & 0 & 0 & 0 \\ 0 & 0 & 0 & 0 & 0 & 0 & 0 & 0 & 0 \\ 0 & 0 & 0 & 0 & 0 & 0 & 0 & 0 & 0,\end{array}$

\begin{tabular}{|c|c|c|c|c|c|c|c|c|c|}
\hline$n * k \quad 1 \quad 0$ & -1 & 0 & O & 0 & -1 & 0 & & & \\
\hline exper* $n^{\star} k$ & 0 & 0 & 0 & 0 & 0 & 0 & 0 & 0 & 0 \\
\hline & 0 & 0 & 0 & 0 & 0 & 0 & 0 & 0 & 0 \\
\hline & 0 & 0 & 0 & 0 & 0 & 0 & 0 & 0 & 0 \\
\hline & 0 & 0 & 0 & 0 & 0 & 0 & 0 & 0 & 0 \\
\hline & 0 & 0 & 0 & 0 & 0 & 0 & 0 & 0 & 0 \\
\hline & 0 & 0 & 0 & 0 & 0 & 0 & 0 & 0 & 0 \\
\hline & 0 & 0 & 0 & 0 & 0 & 0 & 0 & 0 & 0 \\
\hline & 1 & 0 & -1 & 0 & 0 & 0 & -1 & 0 & 1 \\
\hline & 0 & 0 & 0 & 0 & 0 & 0 & 0 & 0 & 0 \\
\hline & 0 & 0 & 0 & 0 & 0 & 0 & 0 & 0 & 0 \\
\hline & 0 & 0 & 0 & 0 & 0 & 0 & 0 & 0 & \\
\hline
\end{tabular}

$\begin{array}{rrrrrrrrrr}\mathrm{n}^{\star} \mathrm{k} 1 & 0 & -1 & 0 & 0 & 0 & -1 & 0 & 1 & \\ \operatorname{exper}^{\star} \mathrm{n}^{\star} \mathrm{k} & 0 & 0 & 0 & 0 & 0 & 0 & 0 & 0 & 0 \\ 0 & 0 & 0 & 0 & 0 & 0 & 0 & 0 & 0 \\ & 0 & 0 & 0 & 0 & 0 & 0 & 0 & 0 & 0 \\ 0 & 0 & 0 & 0 & 0 & 0 & 0 & 0 & 0 \\ 0 & 0 & 0 & 0 & 0 & 0 & 0 & 0 & 0 \\ 0 & 0 & 0 & 0 & 0 & 0 & 0 & 0 & 0 \\ 0 & 0 & 0 & 0 & 0 & 0 & 0 & 0 & 0 \\ 0 & 0 & 0 & 0 & 0 & 0 & 0 & 0 & 0 \\ 1 & 0 & -1 & 0 & 0 & 0 & -1 & 0 & 1 \\ 0 & 0 & 0 & 0 & 0 & 0 & 0 & 0 & 0 \\ 0 & 0 & 0 & 0 & 0 & 0 & 0 & 0 & 0,\end{array}$




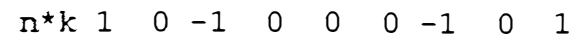

$\begin{array}{rrrrrrrrrr}\text { exper* }{ }^{\star} k & 0 & 0 & 0 & 0 & 0 & 0 & 0 & 0 & 0 \\ 0 & 0 & 0 & 0 & 0 & 0 & 0 & 0 & 0 \\ 0 & 0 & 0 & 0 & 0 & 0 & 0 & 0 & 0 \\ 0 & 0 & 0 & 0 & 0 & 0 & 0 & 0 & 0 \\ 0 & 0 & 0 & 0 & 0 & 0 & 0 & 0 & 0 \\ 0 & 0 & 0 & 0 & 0 & 0 & 0 & 0 & 0 \\ 0 & 0 & 0 & 0 & 0 & 0 & 0 & 0 & 0 \\ 0 & 0 & 0 & 0 & 0 & 0 & 0 & 0 & 0 \\ 0 & 0 & 0 & 0 & 0 & 0 & 0 & 0 & 0 \\ 1 & 0 & -1 & 0 & 0 & 0 & -1 & 0 & 1 \\ 0 & 0 & 0 & 0 & 0 & 0 & 0 & 0 & 0,\end{array}$

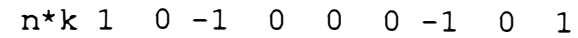

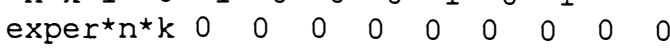

$\begin{array}{rrrrrrrrr}0 & 0 & 0 & 0 & 0 & 0 & 0 & 0 & 0 \\ 0 & 0 & 0 & 0 & 0 & 0 & 0 & 0 & 0 \\ 0 & 0 & 0 & 0 & 0 & 0 & 0 & 0 & 0 \\ 0 & 0 & 0 & 0 & 0 & 0 & 0 & 0 & 0 \\ 0 & 0 & 0 & 0 & 0 & 0 & 0 & 0 & 0 \\ 0 & 0 & 0 & 0 & 0 & 0 & 0 & 0 & 0 \\ 0 & 0 & 0 & 0 & 0 & 0 & 0 & 0 & 0 \\ 0 & 0 & 0 & 0 & 0 & 0 & 0 & 0 & 0 \\ 0 & 0 & 0 & 0 & 0 & 0 & 0 & 0 & 0 \\ 1 & 0 & -1 & 0 & 0 & 0 & -1 & 0 & 1 ;\end{array}$

\begin{tabular}{|c|c|c|c|c|c|c|c|c|c|}
\hline$p \star k \quad 1 \quad 0$ & -1 & 0 & 0 & 0 & -1 & 0 & & & \\
\hline \multirow[t]{11}{*}{ exper ${ }^{\star} p \star k$} & 1 & 0 & -1 & 0 & 0 & 0 & -1 & 0 & 1 \\
\hline & 0 & 0 & 0 & 0 & 0 & 0 & 0 & 0 & 0 \\
\hline & 0 & 0 & 0 & 0 & 0 & 0 & 0 & 0 & 0 \\
\hline & 0 & 0 & 0 & 0 & 0 & 0 & 0 & 0 & 0 \\
\hline & 0 & 0 & 0 & 0 & 0 & 0 & 0 & 0 & 0 \\
\hline & 0 & 0 & 0 & 0 & 0 & 0 & 0 & 0 & 0 \\
\hline & 0 & 0 & 0 & 0 & 0 & 0 & 0 & 0 & 0 \\
\hline & 0 & 0 & 0 & 0 & 0 & 0 & 0 & 0 & 0 \\
\hline & 0 & 0 & 0 & 0 & 0 & 0 & 0 & 0 & 0 \\
\hline & 0 & 0 & 0 & 0 & 0 & 0 & 0 & 0 & 0 \\
\hline & 0 & 0 & 0 & 0 & 0 & 0 & 0 & 0 & \\
\hline
\end{tabular}

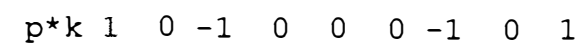

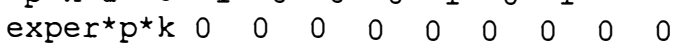
$\begin{array}{rrrrrrrrr}1 & 0 & -1 & 0 & 0 & 0 & -1 & 0 & 1 \\ 0 & 0 & 0 & 0 & 0 & 0 & 0 & 0 & 0\end{array}$ $\begin{array}{lllllllll}0 & 0 & 0 & 0 & 0 & 0 & 0 & 0 & 0\end{array}$ $\begin{array}{lllllllll}0 & 0 & 0 & 0 & 0 & 0 & 0 & 0 & 0\end{array}$ $\begin{array}{lllllllll}0 & 0 & 0 & 0 & 0 & 0 & 0 & 0 & 0\end{array}$ $\begin{array}{lllllllll}0 & 0 & 0 & 0 & 0 & 0 & 0 & 0 & 0\end{array}$ $\begin{array}{lllllllll}0 & 0 & 0 & 0 & 0 & 0 & 0 & 0 & 0\end{array}$ $\begin{array}{lllllllll}0 & 0 & 0 & 0 & 0 & 0 & 0 & 0 & 0\end{array}$ $\begin{array}{lllllllll}0 & 0 & 0 & 0 & 0 & 0 & 0 & 0 & 0 \\ 0 & 0 & 0 & 0 & 0 & 0 & 0 & 0 & 0\end{array}$ 
$p^{\star k} \begin{array}{lllllllll}1 & 0 & -1 & 0 & 0 & 0 & -1 & 0 & 1\end{array}$

\begin{tabular}{|c|c|c|c|c|c|c|c|c|}
\hline exper ${ }^{\star} p^{\star} k 0$ & 0 & 0 & 0 & & & & & \\
\hline 0 & & 0 & 0 & & & & & o \\
\hline & 10 & $0-1$ & 0 & & & & & o \\
\hline 0 & & 0 & 0 & & & & & 0 \\
\hline 0 & & 0 & 0 & & & & & 0 \\
\hline 0 & & 0 & 0 & & & & & 0 \\
\hline 0 & & $\begin{array}{ll}0 & 0\end{array}$ & 0 & & & & & 0 \\
\hline 0 & & 0 & 0 & & & & & 0 \\
\hline 0 & & $\begin{array}{ll}0 & 0\end{array}$ & & & & & & 0 \\
\hline 0 & & $0 \quad 0$ & 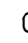 & & & & & 0 \\
\hline 0 & 0 & $0 \quad 0$ & 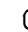 & & & & & 0 \\
\hline
\end{tabular}

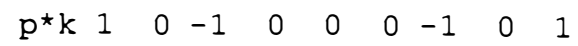

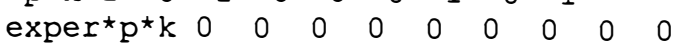
$\begin{array}{lllllllll}0 & 0 & 0 & 0 & 0 & 0 & 0 & 0 & 0 \\ 0 & 0 & 0 & 0 & 0 & 0 & 0 & 0 & 0\end{array}$

$\begin{array}{lllllllll}1 & 0 & -1 & 0 & 0 & 0 & -1 & 0 & 1\end{array}$

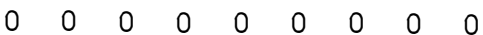

$\begin{array}{lllllllll}0 & 0 & 0 & 0 & 0 & 0 & 0 & 0 & 0\end{array}$

$\begin{array}{lllllllll}0 & 0 & 0 & 0 & 0 & 0 & 0 & 0 & 0\end{array}$

$\begin{array}{lllllllll}0 & 0 & 0 & 0 & 0 & 0 & 0 & 0 & 0\end{array}$

$\begin{array}{lllllllll}0 & 0 & 0 & 0 & 0 & 0 & 0 & 0 & 0\end{array}$

$\begin{array}{lllllllll}0 & 0 & 0 & 0 & 0 & 0 & 0 & 0 & 0 \\ 0 & 0 & 0 & 0 & 0 & 0 & 0 & 0 & 0,\end{array}$

$p^{\star k} \begin{array}{lllllllll}1 & 0 & -1 & 0 & 0 & 0 & -1 & 0 & 1\end{array}$

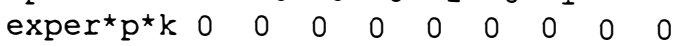
$\begin{array}{lllllllll}0 & 0 & 0 & 0 & 0 & 0 & 0 & 0 & 0 \\ 0 & 0 & 0 & 0 & 0 & 0 & 0 & 0 & 0\end{array}$

$0 \begin{array}{lllllllll}0 & 0 & 0 & 0 & 0 & 0 & 0 & 0 & 0\end{array}$

$\begin{array}{lllllllll}1 & 0 & -1 & 0 & 0 & 0 & -1 & 0 & 1\end{array}$

$\begin{array}{lllllllll}0 & 0 & 0 & 0 & 0 & 0 & 0 & 0 & 0\end{array}$

$\begin{array}{lllllllll}0 & 0 & 0 & 0 & 0 & 0 & 0 & 0 & 0\end{array}$

$\begin{array}{lllllllll}0 & 0 & 0 & 0 & 0 & 0 & 0 & 0 & 0\end{array}$

$\begin{array}{lllllllll}0 & 0 & 0 & 0 & 0 & 0 & 0 & 0 & 0\end{array}$

$\begin{array}{lllllllll}0 & 0 & 0 & 0 & 0 & 0 & 0 & 0 & 0 \\ 0 & 0 & 0 & 0 & 0 & 0 & 0 & 0 & 0,\end{array}$

$\begin{array}{llllllllll}p^{\star} \mathrm{k} & 1 & 0 & -1 & 0 & 0 & 0 & -1 & 0 & 1\end{array}$

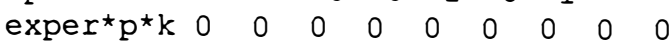

$\begin{array}{lllllllll}0 & 0 & 0 & 0 & 0 & 0 & 0 & 0 & 0 \\ 0 & 0 & 0 & 0 & 0 & 0 & 0 & 0 & 0\end{array}$

$\begin{array}{lllllllll}0 & 0 & 0 & 0 & 0 & 0 & 0 & 0 & 0\end{array}$

$\begin{array}{lllllllll}0 & 0 & 0 & 0 & 0 & 0 & 0 & 0 & 0\end{array}$

$\begin{array}{lllllllll}1 & 0 & -1 & 0 & 0 & 0 & -1 & 0 & 1\end{array}$

$\begin{array}{lllllllll}0 & 0 & 0 & 0 & 0 & 0 & 0 & 0 & 0\end{array}$

$\begin{array}{lllllllll}0 & 0 & 0 & 0 & 0 & 0 & 0 & 0 & 0\end{array}$

$\begin{array}{lllllllll}0 & 0 & 0 & 0 & 0 & 0 & 0 & 0 & 0\end{array}$

$\begin{array}{lllllllll}0 & 0 & 0 & 0 & 0 & 0 & 0 & 0 & 0 \\ 0 & 0 & 0 & 0 & 0 & 0 & 0 & 0 & 0\end{array}$ 


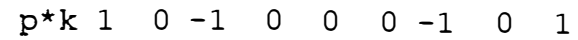

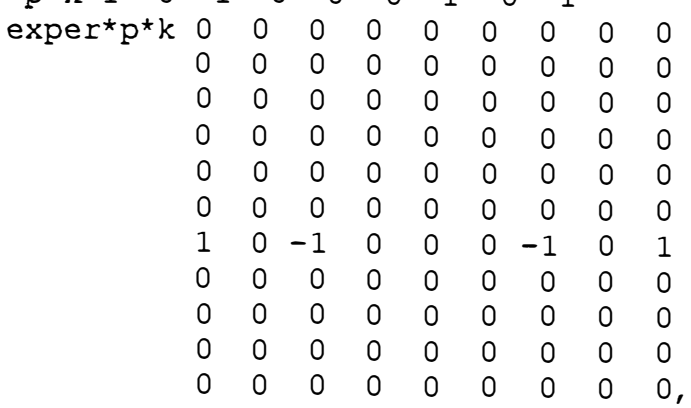

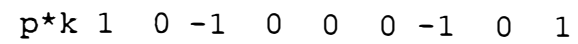

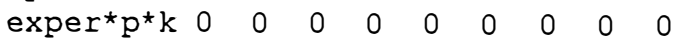
$\begin{array}{lllllllll}0 & 0 & 0 & 0 & 0 & 0 & 0 & 0 & 0\end{array}$ $\begin{array}{lllllllll}0 & 0 & 0 & 0 & 0 & 0 & 0 & 0 & 0\end{array}$

$\begin{array}{lllllllll}0 & 0 & 0 & 0 & 0 & 0 & 0 & 0 & 0\end{array}$

$\begin{array}{lllllllll}0 & 0 & 0 & 0 & 0 & 0 & 0 & 0 & 0\end{array}$

$\begin{array}{lllllllll}0 & 0 & 0 & 0 & 0 & 0 & 0 & 0 & 0\end{array}$

$\begin{array}{lllllllll}0 & 0 & 0 & 0 & 0 & 0 & 0 & 0 & 0\end{array}$

$\begin{array}{lllllllll}1 & 0 & -1 & 0 & 0 & 0 & -1 & 0 & 1\end{array}$

$\begin{array}{lllllllll}0 & 0 & 0 & 0 & 0 & 0 & 0 & 0 & 0\end{array}$

$\begin{array}{lllllllll}0 & 0 & 0 & 0 & 0 & 0 & 0 & 0 & 0 \\ 0 & 0 & 0 & 0 & 0 & 0 & 0 & 0 & 0\end{array}$

$p * k \begin{array}{lllllllll}1 & 0 & -1 & 0 & 0 & 0 & -1 & 0 & 1\end{array}$

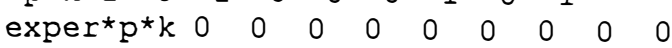

$\begin{array}{lllllllll}0 & 0 & 0 & 0 & 0 & 0 & 0 & 0 & 0\end{array}$

$\begin{array}{lllllllll}0 & 0 & 0 & 0 & 0 & 0 & 0 & 0 & 0\end{array}$

$\begin{array}{lllllllll}0 & 0 & 0 & 0 & 0 & 0 & 0 & 0 & 0\end{array}$

$\begin{array}{lllllllll}0 & 0 & 0 & 0 & 0 & 0 & 0 & 0 & 0\end{array}$

$\begin{array}{lllllllll}0 & 0 & 0 & 0 & 0 & 0 & 0 & 0 & 0\end{array}$

$\begin{array}{lllllllll}0 & 0 & 0 & 0 & 0 & 0 & 0 & 0 & 0\end{array}$

$\begin{array}{lllllllll}0 & 0 & 0 & 0 & 0 & 0 & 0 & 0 & 0\end{array}$

$\begin{array}{lllllllll}1 & 0 & -1 & 0 & 0 & 0 & -1 & 0 & 1\end{array}$

$\begin{array}{lllllllll}0 & 0 & 0 & 0 & 0 & 0 & 0 & 0 & 0 \\ 0 & 0 & 0 & 0 & 0 & 0 & 0 & 0 & 0\end{array}$

$p^{* k} \begin{array}{lllllllll}1 & 0 & -1 & 0 & 0 & 0 & -1 & 0 & 1\end{array}$

$\begin{array}{rrrrrrrrrr}\text { exper* } p^{\star} k & 0 & 0 & 0 & 0 & 0 & 0 & 0 & 0 & 0 \\ 0 & 0 & 0 & 0 & 0 & 0 & 0 & 0 & 0 \\ 0 & 0 & 0 & 0 & 0 & 0 & 0 & 0 & 0 \\ 0 & 0 & 0 & 0 & 0 & 0 & 0 & 0 & 0 \\ 0 & 0 & 0 & 0 & 0 & 0 & 0 & 0 & 0 \\ 0 & 0 & 0 & 0 & 0 & 0 & 0 & 0 & 0 \\ 0 & 0 & 0 & 0 & 0 & 0 & 0 & 0 & 0 \\ 0 & 0 & 0 & 0 & 0 & 0 & 0 & 0 & 0 \\ 0 & 0 & 0 & 0 & 0 & 0 & 0 & 0 & 0 \\ 1 & 0 & -1 & 0 & 0 & 0 & -1 & 0 & 1 \\ 0 & 0 & 0 & 0 & 0 & 0 & 0 & 0 & 0,\end{array}$


$p^{* k} \quad \begin{array}{lllllllll}1 & 0 & -1 & 0 & 0 & 0 & -1 & 0 & 1\end{array}$

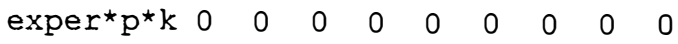

$\begin{array}{lllllllll}0 & 0 & 0 & 0 & 0 & 0 & 0 & 0 & 0\end{array}$

$\begin{array}{lllllllll}0 & 0 & 0 & 0 & 0 & 0 & 0 & 0 & 0\end{array}$

$\begin{array}{lllllllll}0 & 0 & 0 & 0 & 0 & 0 & 0 & 0 & 0\end{array}$

$\begin{array}{lllllllll}0 & 0 & 0 & 0 & 0 & 0 & 0 & 0 & 0\end{array}$

$\begin{array}{lllllllll}0 & 0 & 0 & 0 & 0 & 0 & 0 & 0 & 0\end{array}$

$\begin{array}{lllllllll}0 & 0 & 0 & 0 & 0 & 0 & 0 & 0 & 0\end{array}$

$\begin{array}{lllllllll}0 & 0 & 0 & 0 & 0 & 0 & 0 & 0 & 0\end{array}$

$\begin{array}{lllllllll}0 & 0 & 0 & 0 & 0 & 0 & 0 & 0 & 0\end{array}$

$\begin{array}{rrrrrrrrr}0 & 0 & 0 & 0 & 0 & 0 & 0 & 0 & 0 \\ 1 & 0 & -1 & 0 & 0 & 0 & -1 & 0 & 1\end{array}$

run;

quit; 


\section{APÊNDICE 3}

Programa SAS para análise de variância ponderada 


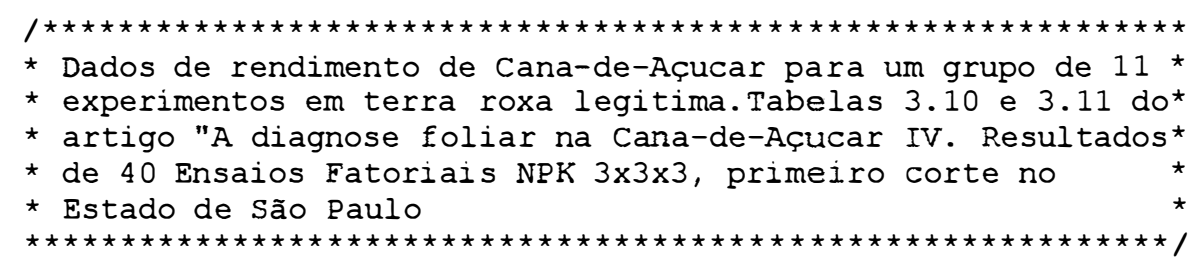

libname wil 'c:l';

options nodate nonumber $1 \mathrm{~s}=75 \mathrm{ps}=80$;

data wil.grupo;

do exper $=1$ to 11 ;

do $\mathrm{n}=0$ to 2 ;

do $\mathrm{p}=0$ to 2 ;

do $k=0$ to 2 ;

input rend @@;

output;

end;

end;

end;

end;

cards;

$\begin{array}{lllllllll}130.5 & 117.0 & 122.8 & 135.2 & 146.4 & 142.5 & 118.2 & 146.4 & 113.9\end{array}$

$\begin{array}{lllllllll}129.8 & 148.2 & 149.5 & 143.6 & 131.4 & 136.2 & 109.3 & 130.7 & 156.2\end{array}$

$\begin{array}{lllllllll}124.5 & 137.7 & 120.7 & 126.8 & 146.6 & 138.0 & 134.5 & 139.3 & 133.7\end{array}$

$\begin{array}{lllllllll}113.9 & 127.5 & 131.2 & 120.0 & 140.5 & 133.4 & 115.7 & 132.0 & 114.5\end{array}$

$\begin{array}{lllllllll}139.3 & 127.8 & 133.4 & 141.6 & 144.5 & 145.0 & 131.2 & 151.8 & 146.8\end{array}$

$\begin{array}{lllllllll}142.7 & 162.0 & 153.7 & 147.0 & 145.0 & 166.8 & 133.2 & 139.5 & 153.4\end{array}$

$\begin{array}{lllllllll}65.7 & 91.2 & 98.2 & 65.7 & 88.9 & 107.7 & 67.8 & 90.1 & 95.9\end{array}$

$\begin{array}{lllllllll}61.1 & 88.7 & 104.8 & 75.3 & 120.9 & 105.3 & 72.3 & 101.6 & 104.8\end{array}$

$\begin{array}{lllllllll}76.1 & 89.8 & 114.3 & 61.1 & 98.9 & 111.8 & 110.3 & 100.1 & 121.4\end{array}$

$\begin{array}{lllllllll}43.4 & 95.5 & 96.1 & 92.3 & 88.7 & 88.7 & 68.7 & 72.7 & 122.8\end{array}$

$\begin{array}{lllllllll}56.2 & 72.3 & 113.4 & 78.2 & 91.6 & 115.9 & 71.4 & 101.6 & 96.6\end{array}$

$\begin{array}{lllllllll}74.2 & 98.6 & 107.1 & 63.4 & 110.7 & 116.6 & 66.4 & 111.2 & 116.4\end{array}$

$\begin{array}{llllllllll}265.2 & 246.1 & 269.6 & 273.9 & 273.6 & 233.4 & 288.9 & 244.5 & 281.8\end{array}$

$\begin{array}{llllllllll}257.7 & 273.7 & 260.5 & 225.0 & 237.1 & 274.6 & 265.0 & 239.3 & 239.3\end{array}$

$\begin{array}{lllllllll}263.6 & 283.4 & 285.0 & 237.1 & 261.2 & 271.4 & 256.6 & 280.2 & 254.1\end{array}$

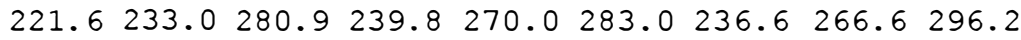

$\begin{array}{lllllllll}298.6 & 260.2 & 266.0 & 241.6 & 230.5 & 252.1 & 227.5 & 246.2 & 292.3\end{array}$

$\begin{array}{llllllllll}235.2 & 300.2 & 255.7 & 243.6 & 293.9 & 273.9 & 251.6 & 273.9 & 289.8\end{array}$

$\begin{array}{llllllllll}252.0 & 236.4 & 282.7 & 245.7 & 252.1 & 267.7 & 202.1 & 239.3 & 254.5\end{array}$

$\begin{array}{lllllllll}218.4 & 269.0 & 251.4 & 255.0 & 248.9 & 272.5 & 257.0 & 249.5 & 254.5\end{array}$

$\begin{array}{llllllllll}264.3 & 264.8 & 256.4 & 248.4 & 256.8 & 243.0 & 264.0 & 257.0 & 276.8\end{array}$

$\begin{array}{lllllllll}70.5 & 117.8 & 109.5 & 147.0 & 96.6 & 131.2 & 101.8 & 101.6 & 122.7\end{array}$

$\begin{array}{lllllllll}129.5 & 106.2 & 137.0 & 118.9 & 105.2 & 112.7 & 139.1 & 130.5 & 124.8\end{array}$

$\begin{array}{lllllllll}120.9 & 113.2 & 110.0 & 134.6 & 115.2 & 128.7 & 90.3 & 127.7 & 152.0\end{array}$

$\begin{array}{lllllllll}220.2 & 202.7 & 176.2 & 160.5 & 175.9 & 226.1 & 228.7 & 254.5 & 191.2\end{array}$

$\begin{array}{lllllllll}228.6 & 256.8 & 228.2 & 269.6 & 219.3 & 224.8 & 254.6 & 220.9 & 220.3\end{array}$

$\begin{array}{llllllllll}162.3 & 221.8 & 212.7 & 235.9 & 233.9 & 230.9 & 216.8 & 207.5 & 230.5\end{array}$

$\begin{array}{lllllllll}95.0 & 138.2 & 146.1 & 152.5 & 167.5 & 150.7 & 130.9 & 170.3 & 157.3\end{array}$

$\begin{array}{lllllllll}144.5 & 150.7 & 169.8 & 130.9 & 125.7 & 133.7 & 158.4 & 175.2 & 142.8\end{array}$

$\begin{array}{lllllllll}144.5 & 173.6 & 141.1 & 134.5 & 150.5 & 145.5 & 144.6 & 146.6 & 153.2\end{array}$ $\begin{array}{llllllllll}148.9 & 163.1 & 165.9 & 215.6 & 185.7 & 193.7 & 148.7 & 183.0 & 169.9\end{array}$ $\begin{array}{lllllllll}188.9 & 187.8 & 194.5 & 164.3 & 155.6 & 162.5 & 172.0 & 163.3 & 169.5\end{array}$ $\begin{array}{llllllllll}153.2 & 156.4 & 195.9 & 137.1 & 174.6 & 155.6 & 173.7 & 197.7 & 194.3\end{array}$

; 


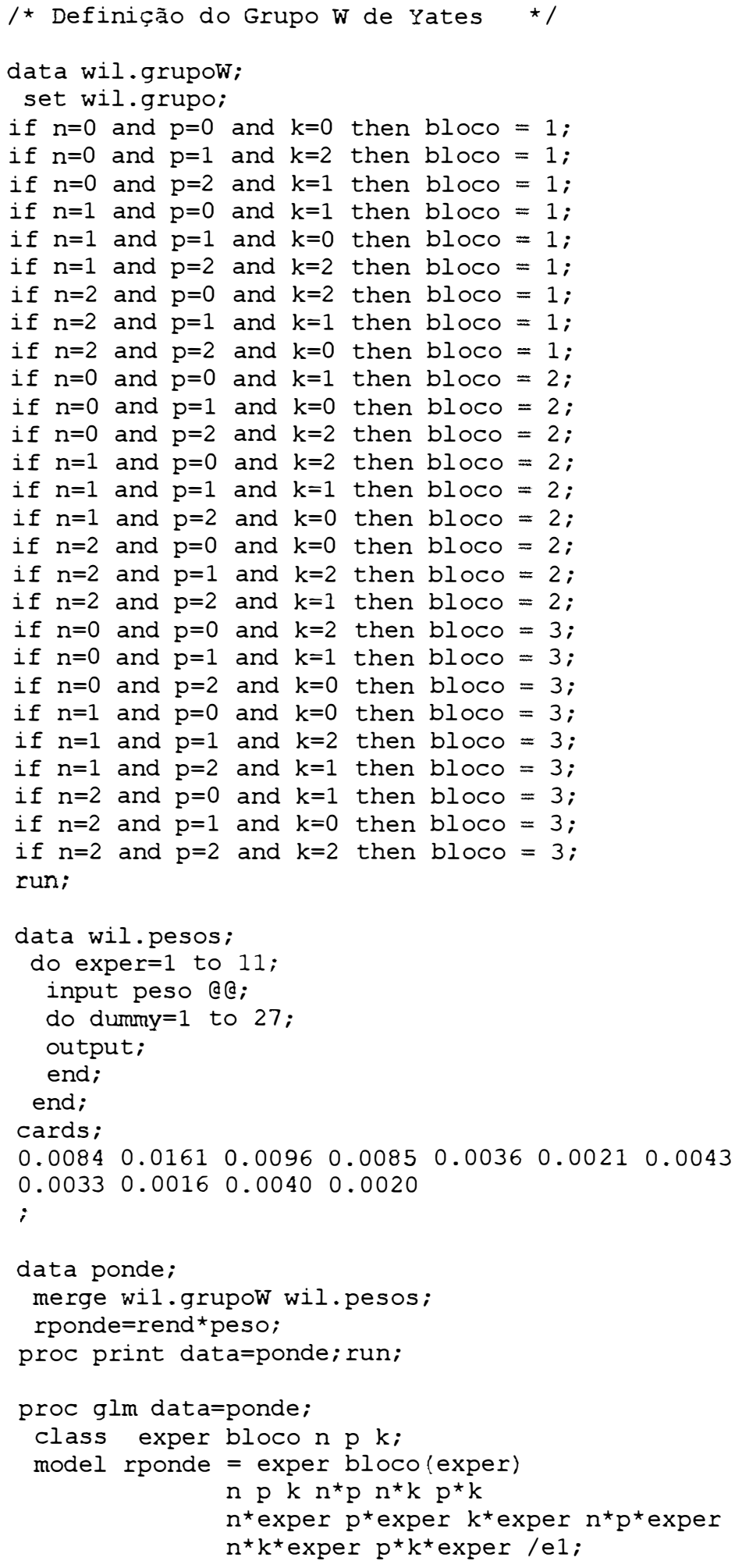




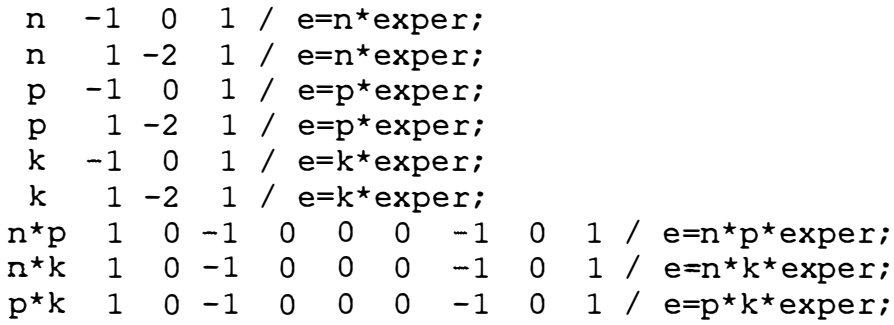

Obs: O restante do programa é idêntico ao programa para análise de variância não ponderada (apêndice 2). 